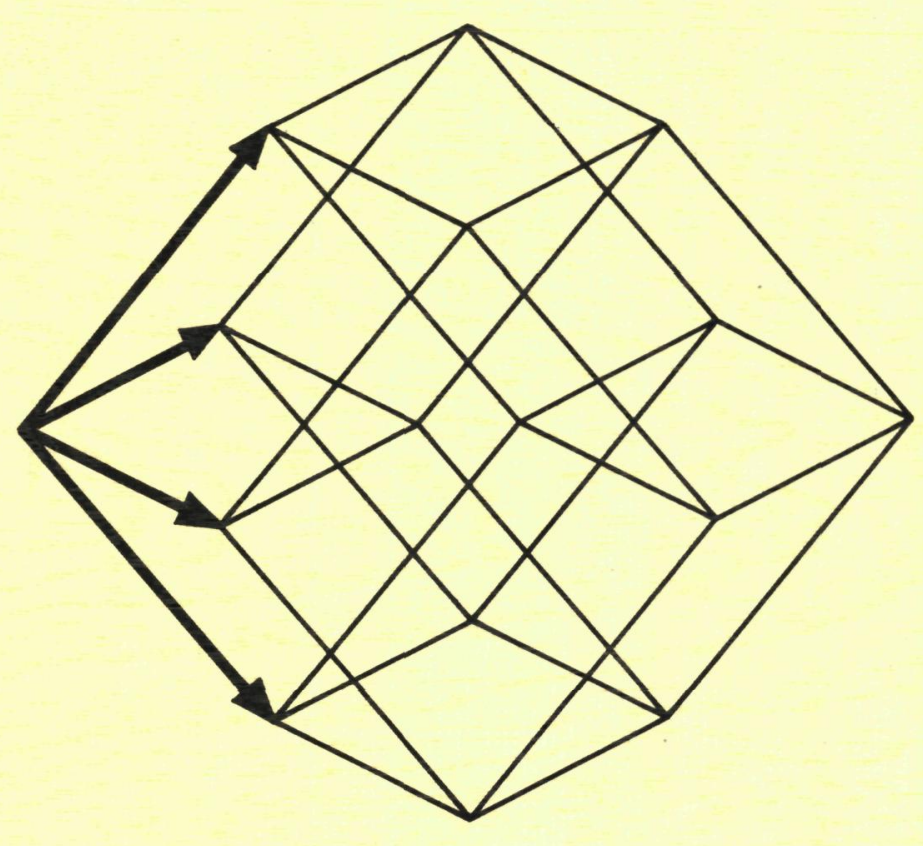

t. janssen

\title{
crystallographic groups in space and time
}
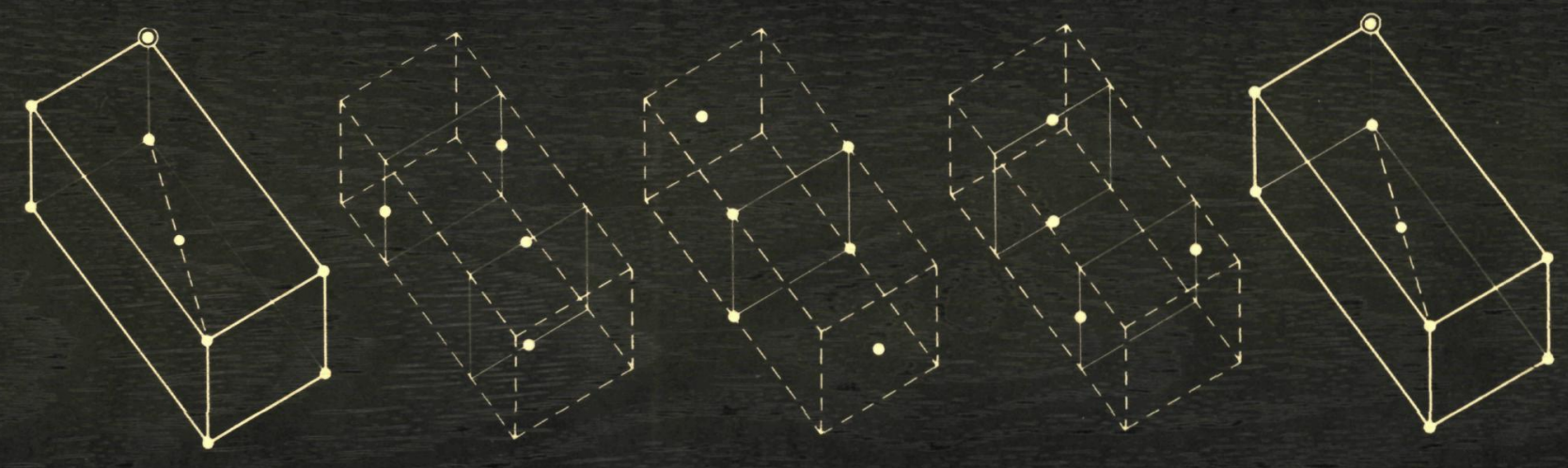



\section{crystallographic groups in space and time}


PROMOTOR: PROF.DR. A.G.M. JANNER 


\title{
crystallographic groups in space and time
}

\author{
PROEFSCHRIFT \\ TER VERKRIJGING VAN DE GRAAD VAN DOCTOR \\ IN DE WISKUNDE EN NATUURWETENSCHAPPEN \\ AAN DE KATHOLIEKE UNIVERSITEIT TE NIJMEGEN. \\ OP GEZAG VAN DE RECTOR MAGNIFICUS DR. A.Th. L.M. MERTENS, \\ HOOGLERAAR IN DE FACULTEIT DER GENEESKUNDE. \\ VOLGENS BESLUIT VAN DE SENAAT \\ IN HET OPENBAAR TE VERDEDIGEN \\ OP VRIJDAG 14 JUNI 1968 DES NAMIDDAGS TE 2 UUR
}

DOOR

THEO WILLEM JAN MARIE JANSSEN GEBOREN TE VUGHT 

Op deze plaats wil ik graag allen bedanken die hebben meegewerkt aan de totstandkoming van dit proefschrift. Naast diegenen die op grond van artikel 7 van de promotieregeling niet genoemd mogen worden, wil ik nog de volgende personen mijn erkentelijkheid betuigen.

I would like to thank prof. M. Newman and prof. J. Mennicke for helpful discussions.

Herm dr. J. Neubüser und Dipl. Math. R. Bülow schulde ich Dank für Ihre Hilfe beim kontrollieren der Kristallklassen.

Je veux remercier dr. E. Ascher pour ses remarques.

Dr. W. Tam was so kind to look at the manuscript and to correct several mistakes against the English language.

Mijn dank gaat ook uit naar mej. H. Bijmans, die het typewerk met grote snelheid voltooide. De illustraties en omslag werden verzorgd door de afdeling Illustratie o.l.v. de Heer J. Gerritsen. Het drukken gebeurde op de Offsetdrukkerij van de Faculteit o.l.v. de Heer Th.A. Gunsing. 

Voor Loes

Voor Moeder 


\section{CONTENTS}

Part I. General definitions and basic properties

1. Introduction 1

2. Metric spaces and transformation groups 3

3. Euclidean space groups 9

4. Relativistic crystallographic groups 17

5. Galilean crystallographic groups 24

6. Conclusion 30

Part II. Central extensions

1. Introduction 32

2. The determination of $\mathrm{H}_{\phi}^{2}(K, A) \quad 33$

3. Extensions of $z^{n} \quad 38$

4. The connection between the cohomology groups $\mathrm{H}_{\phi}^{2}\left(\mathrm{~K}, \mathrm{z}^{\mathrm{n}}\right)$ and $H_{\phi}^{l}\left(K, R^{n} / Z^{n}\right) \quad 42$

5. Relation between the vectors $U$ and $\phi$, the factor system $m$ and the system of non-primitive translations $u$

6. Isomorphism 53

7. Example 59

8. Conclusion 67

Appendix: Extension conditions for groups isomorphic to four-dimensional GM point groups 68

Part III. Four-dimensional Euclidean crystal classes corresponding to GM point groups

1. Introduction 71

2. GM point groups 72

3. Lattices in Euclidean space 77

4. Four-dimensional Bravais classes and arithmetic crystal classes corresponding to GM point groups 84

$\begin{array}{ll}\text { Tables } & 88\end{array}$

$\begin{array}{ll}\text { Samenvatting } & 95\end{array}$

$\begin{array}{ll}\text { Summary } & 97\end{array}$

$\begin{array}{ll}\text { References } & 99\end{array}$ 


\section{PART ONE: GENERAL DEFINITIONS AND BASIC PROPERTIES}

\section{Introduction}

In recent years an extensive study has been made of magnetic groups [1], [2]. These are n-dimensional crystallographic groups in which time inversion is considered next to and in combination with space transformations.

If one admits also discrete time translations one is led to the study of $n+1$-dimensional crystallographic groups. The problem is to find a suitable space on which these groups act as transformation groups. Consider the simplest possibility: a $n+1$-dimensional Euclidean vector space which is a vector space with a positive definite metric. The group of inhomogeneous real linear transformations leaving this metric invariant is the Euclidean group $E(n+1)$. A $n+1$-dimensional space group is a discrete subgroup of the Euclidean group which contains as a maximal abelian subgroup a translation group generating a $n+1$-dimensional lattice. As Ascher and Janner [3] have discussed, such a space group $G^{n+1}$ may be obtained from an extension:

$$
0 \rightarrow \mathrm{z}^{\mathrm{n}+1} \rightarrow \mathrm{G}^{\mathrm{n}+1} \rightarrow \mathrm{K} \rightarrow 1
$$

of a free abelian group $\mathrm{z}^{\mathrm{n}+1}$ by a finite group $\mathrm{K}$ called point group. The operation of $K$ on $\mathrm{z}^{\mathrm{n}+1}$ is given by a monomorphism $\phi: K \rightarrow G L(n+1, z)$. In crystallography it is customary to identify affine equivalent space groups. According to Bieberbach [4] two space groups are affine equivalent if and only if they are isomorphic.

However the Euclidean space does not take into account the difference between time axis and space axes. The solution of the problem would be considering the $n+1$-dimensional Minkowskian vector space which has an indefinite metric of signature $(n, 1)$. The homogeneous real linear group leaving this metric invariant is the Lorentz group, the inhomogeneous one is the Poincare group. A relativistic space-time group is a discrete subgroup of the Poincaré group with a maximal abelian subgroup of translations generating a $n+1-$ 
dimensional lattice. Relativistic space-time groups can also be obtained from extensions (1.1), but the general conditions under which groups appearing in extensions (1.1) may be interpreted as relativistic space-time groups are under investigation. Because in Minkowskian space one has to distinguish between spacelike, timelike and isotropic vectors, equivalence of two spacetime groups is a stronger equivalence relation than plain isomorphism as groups. We call two such groups isomorphic if there exists an isomorphism between them which maps translations on translations of the same kind. To find all non-isomorphic relativistic space-time groups is, as far as we know, an unsolved problem even in the lowest dimension $(n=1)$. This is a consequence of the indefinite character of the metric.

In the non-relativistic limit the Minkowskian vector space is transformed into the Galilean vector space with a singular metric. In this same limit the Poincaré group becomes the inhomogeneous Galilean group. A Galilean space-time group is a discrete subgroup of the inhomogeneous Galilean group with a maximal abelian subgroup of translations generating a $n+1$-dimensional lattice. In Galilean space one distinguishes space type vectors $\left(n+1^{\text {th }}\right.$ component zero) and velocity type vectors ( $n+1^{\text {th }}$ component different from zero). Two Galilean space-time groups are called isomorphic if there is a group isomorphism between them which maps translation elements on translations of the same kind.

Regarding the difficulties involved in the determination of relativistic and Galilean space-time groups it is worthwhile to consider a last possibility: the direct product of a n-dimensional Euclidean space (interpreted as space) and a one-dimensional Euclidean space (representing the time). We simply call it product space. Actually this is the type of vector space one considers implicitly when speaking of magnetic groups. The group of inhomogeneous linear transformations leaving invariant the metric in both spaces is called the inhomogeneous pseudo-Lorentz group. A generalized magnetic spacetime group (GM space-time group) is a discrete subgroup of the inhomogeneous pseudo-Lorentz group JP $(n+1)$ with a maximal abelian subgroup of translations generating a $n+1$-dimensional lattice. In product space one may distinguish three kinds of vectors: those lying in one of the two spaces and a mixed type (with non-vanishing components in both spaces). Two GM space-time groups are called isomorphic if there exists a group isomorphism between them mapping translation elements of a given type on translation elements of the same type. 
The present part presents a study of the properties and the classification of the space-(time) groups in the three first named different spaces. The classification of GM space-time groups is not further treated, but this may be done quite analogously. Some preliminary results are published in technical reports [5].

Before starting the discussion of the various crystallographic groups a brief survey of the corresponding continuous transformation groups is given.

\section{Metric spaces and transformation groups}

Consider a $n+1$-dimensional real vector space $\mathrm{R}^{\mathrm{n}+1}$. Provided with a positive definite metric this is a Euclidean space. (If an orthonormal basis $e_{1}, e_{2}, \ldots, e_{n+1}$ is chosen a vector $x \in R^{n+1}$ has real components $\left(x^{1}, \ldots, x^{n+1}\right)$ and its norm is given by the non-degenerate quadratic form

$$
\left.\|x\|^{2}=\sum_{i=1}^{n+1}\left(x^{i}\right)^{2}\right) .
$$

The non-singular linear transformations leaving this metric invariant form the orthogonal group $O(n+1)$. The inhomogeneous transformations leaving the norm of the difference of any two vectors invariant form the group of solid motions, the Euclidean group $E(n+1)$. The uniquely determined maximal abelian normal subgroup of $E(n+1)$ is the $n+1$-dimensional translation group $T^{n+1}$. $E(n+1)$ is the semi-direct product

$$
E(n+1)=T^{n+1} \wedge O(n+1)
$$

where $0(n+1)$ acts on $T^{n+1}$ in the natural way. Denoting the elements of $E(n+1)$ by $(a, \alpha)$, where $a \in T^{n+1}$ and $\alpha \in O(n+1)$, multiplication in $E(n+1)$ is defined by

$$
(a, \alpha)(b, \beta)=(a+\alpha b, \alpha \beta)
$$

for all $a, b \in T^{n+1}$ and $a l l a, \beta \in O(n+1)$. By $\alpha b$ is meant $\phi(\alpha) b$, where $\phi$ is here the natural monomorphism $O(n+1) \rightarrow \operatorname{Aut}\left(T^{n+1}\right)$. 
Providing $R^{n+1}$ with an indefinite metric of signature $(n, 1)$ one has a $n+1-$ dimensional Minkowskian space. (Choosing an orthonormal basis $e_{1}, \ldots, e_{n+1}$ in such a way that

$$
\begin{aligned}
& \left\|e_{i}\right\|^{2}=1 \quad(i=1, \ldots, n) \\
& \left\|e_{n+1}\right\|^{2}=-1
\end{aligned}
$$

the norm of a vector with components $x^{1}, \ldots, x^{n+1}$ is given by

$$
\left.\|x\|^{2}=\sum_{i=1}^{n}\left(x^{i}\right)^{2}-\left(x^{n+1}\right)^{2}\right)
$$

The orthogonal group of this metric is the homogeneous Lorentz group $O(n, 1)$. The inhomogeneous Lorentz group or Poincare group $J L(n+1)$ is the semi-direct product

$$
J L(n+1)=T^{n+1} \wedge O(n, 1)
$$

where $O(n, 1)$ operates on $\mathrm{T}^{\mathrm{n}+1}$ in the natural way.

By choosing another orthogonal basis in Minkowskian space defined by

$$
\begin{array}{ll}
e_{i}^{\prime}=e_{i} & (i=1, \ldots, n) \\
e_{n+1}^{\prime}=c e_{n+1} & (\text { real positive c) }
\end{array}
$$

one obtains in the limit $c \rightarrow \infty$ the $n+1$-dimensional Galilean space with a singular metric. The corresponding limit of the Lorentz group is the Galilean group $G(n+1)$ which is obtained in the following way. By $0^{+}(n, 1)$ we denote the one-component of $O(n, 1)$ and by $V$ :

- for odd $n$ : the group generated by

$$
\left(\begin{array}{cc}
-\mathbb{1}_{n} & 0 \\
0 & 1
\end{array}\right) \quad \text { and } \quad\left(\begin{array}{cc}
\mathbb{1}_{\mathrm{n}} & 0 \\
0 & -1
\end{array}\right)
$$

- for even $\mathrm{n}$ : the group generated by 


$$
\left(\begin{array}{cc}
\mathbb{1}_{n} & 0 \\
0 & -1
\end{array}\right) \quad \text { and } \quad\left(\begin{array}{cc}
M_{n} & 0 \\
0 & 1
\end{array}\right) \text { with } M_{n}=\left(\begin{array}{cc}
-1 & 0 \\
0 & \mathbb{1}_{n-1}
\end{array}\right)
$$

Then $O(n, 1)$ is generated by $0^{+}(n, 1)$ and $V$. Any $\lambda \in 0^{+}(n, 1)$ may be written as:

$$
\lambda(x)=\left(\begin{array}{cc}
P_{n} & \vdots \\
& 0 \\
0 \ldots 0 & 1
\end{array}\right)\left(\begin{array}{ccc}
\cosh x & 0 & \sinh x \\
0 & \mathbb{1}_{n-1} & 0 \\
\sinh x & 0 & \cosh x
\end{array}\right)\left(\begin{array}{cc}
Q_{n} & \vdots \\
& 0 \\
0 \ldots 0 & 1
\end{array}\right)
$$

where $P_{n}$ and $Q_{n}$ are $n$-dimensional orthogonal transformations. After the basis transformation (2.1) and putting $|v|=c x$ one has:

$$
\lim _{c \rightarrow \infty} \lambda \cdot\left(\frac{v}{c}\right)=\left(\begin{array}{cc}
P_{n}{ }^{Q} n & v \\
0 & 1
\end{array}\right) \equiv \gamma(v)
$$

where $v$ is a n-dimensional columnvector. $\gamma(v)$ is an element of $G(n+1)$. $G(n+1)$ is generated by what we denote as $\lim _{c \rightarrow \infty} O^{+}(n, 1)$ and $V$.

The inhomogeneous Galilean group is the semi-direct product

$$
J G(n+1)=T^{n+1} \wedge G(n+1)
$$

where again $G(n+1)$ acts on $T^{n+1}$ in the natural way.

We call product space the direct product of a n-dimensional and a onedimensional Euclidean space. The metric in the first space is given by:

$$
x^{2}=\sum_{i=1}^{n}\left(x^{i}\right)^{2}
$$

in the second one by

$$
x^{2}=\left(x^{n+1}\right)^{2}
$$

The linear transformations leaving invariant the metric in both spaces form 
the homogeneous pseudo-Lorentz group $\underline{Q}(n, 1)$.

Proposition 1

$$
\underline{O}(n, 1)=O(n) \times O(1) .
$$

Proof: Choose an orthonormal basis with $e_{1}, \ldots, e_{n}$ in the first space and $e_{n+1}$ in the second one. Then an element $T \in \underline{O}(n, 1)$ is a matrix such that for any $x$ with components $x^{1}, \ldots, x^{n+1}$ :

$$
\sum_{i=1}^{n}\left(x^{i}\right)^{2}=\sum_{j=1}^{n} \sum_{k, \ell=1}^{n+1} T_{j k} T_{j \ell} x^{k} x^{\ell}
$$

and

$$
\left(x^{n+1}\right)^{2}=\sum_{k, \ell=1}^{n+1} T_{n+1, k} T_{n+1, \ell} x^{k} x^{\ell}
$$

From this follows

$$
\begin{array}{ll}
\sum_{j=1}^{n} T_{j k} T_{j l}=\delta_{k l} & (k, l \leq n) \\
T_{j, n+1}=T_{n+1, k}=0 & (j, k \leqslant n) \\
T_{n+1, n+1}^{2}=1 . &
\end{array}
$$

Therefore the matrix $\mathrm{T}$ has the form

$$
T=\left(\begin{array}{cc}
P & 0 \\
& \\
0 & \pm 1
\end{array}\right)
$$

where $P$ is an orthogonal matrix.

\section{Proposition 2}

The pseudo-Lorentz group leaves invariant all quadratic forms 


$$
\alpha \sum_{i=1}^{n}\left(x^{i}\right)^{2}+\beta\left(x^{n+1}\right)^{2} \quad(\text { all real } \alpha \text { and } \beta)
$$

This is a direct consequence of proposition 1.

In particular $\underline{O}(n, 1)$ leaves invariant the quadratic forms

$$
\sum_{i=1}^{n+1}\left(x^{i}\right)^{2} \text { and } \sum_{i=1}^{n}\left(x^{i}\right)^{2}-\left(x^{n+1}\right)^{2}
$$

The inhomogeneous pseudo-Lorentz group $\mathrm{JP}(\mathrm{n}+1)$ is the semi-direct product:

$$
J P(n+1)=T^{n+1} \wedge \underline{o}(n, 1) .
$$

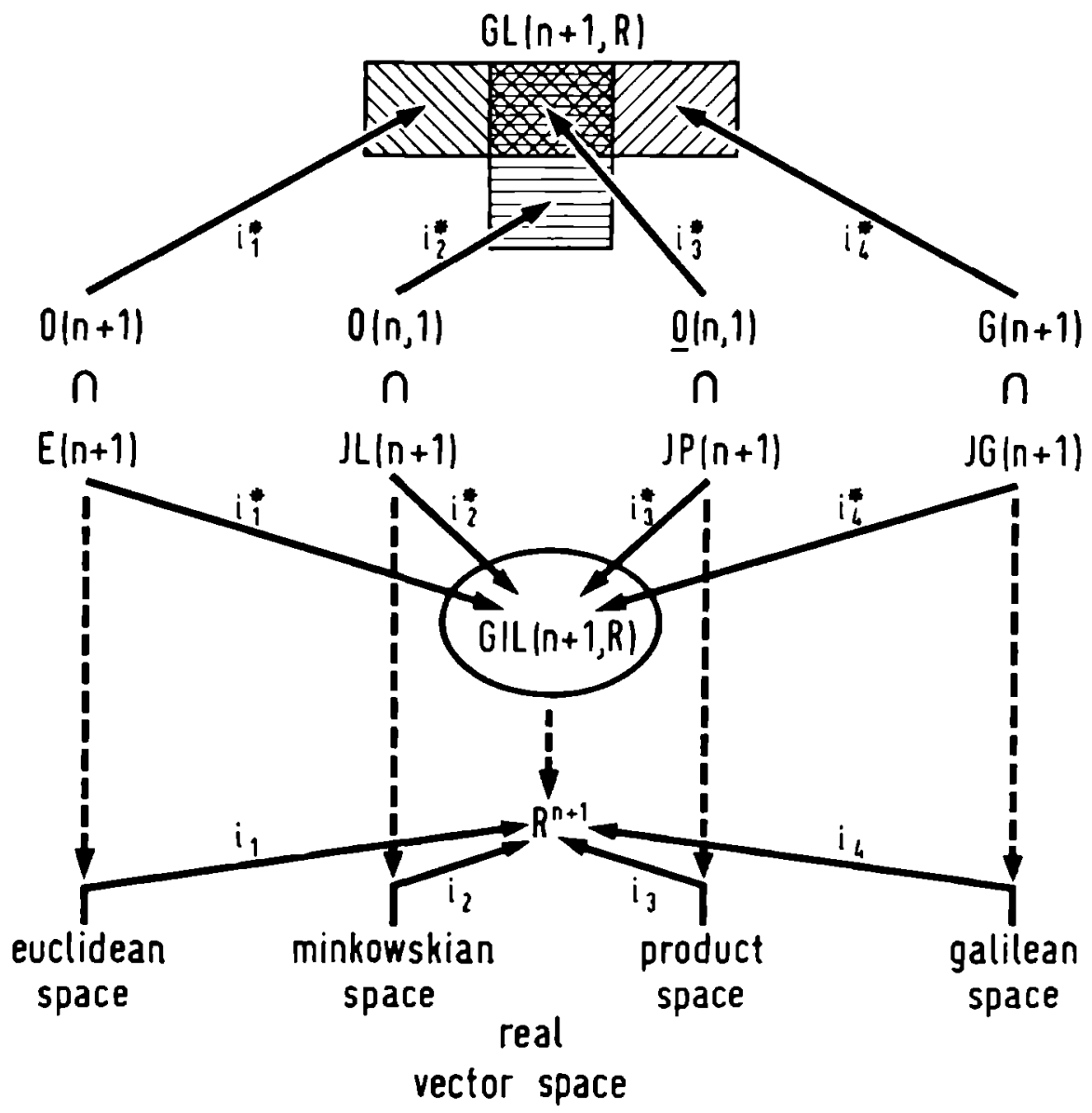

fig. 1 
By choosing a basis in each of the spaces discussed one obtains isomorphic mappings onto $\mathrm{R}^{\mathrm{n}+1}$ considered as real vector space. These mappings induce isomorphic mappings of the groups $E(n+1), J L(n+1), J P(n+1)$ and $J G(n+1)$ into the real affine group $G I L(n+1, R)$, which is the semi-direct product of $R^{n+1}$ by the real general linear group $G L(n+1, R)$.

For the rest of the paper we suppose that a fixed orthonormal basis is chosen, if not stated otherwise. As $n+1^{\text {th }}$ axis we take:

- in Minkowskian space the direction of the basis vector of norm -1;

- in Galilean space the direction obtained in its limit (2.1);

- in product space the one-dimensional Euclidean space.

The $n+1^{\text {th }}$ axis is sometimes called t-axis.

Once these bases are chosen the transformation groups may be identified with their isomorphic images in $\operatorname{GIL}(n+1, R)$.

Proposition 3:

$$
\underline{O}(n, 1)=O(n+1) \cap O(n, 1)=0(n+1) \cap G(n+1)=O(n, 1) \cap G(n+1)
$$

The proof is a direct consequence of proposition 1 and the fact that the elements of $G(n+1)$ have the matrix form

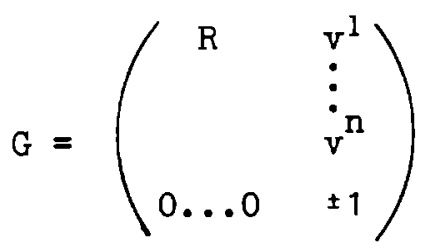

where $R \in O(n)$.

Because in each space the group of solid motions is the semi-direct product of the translation group by the orthogonal group of the space one has: Proposition 4:

$$
\begin{aligned}
J P(n+1) & =E(n+1) \cap J L(n+1) \\
& =E(n+1) \cap J G(n+1) \\
& =J L(n+1) \cap J G(n+1) .
\end{aligned}
$$




\section{Euclidean space groups}

We give here a brief summary of those properties of Euclidean space groups which form a fundament for the subsequent generalizations. At the same time we introduce some new concepts. We refer to references [3], [4], [5] for more details and for the proofs.

A $\mathrm{n}+1$-dimensional space group $G^{\mathrm{n}+1}$ is a subgroup of the Euclidean group $E(n+1)$ with $U^{n+1} \stackrel{\text { def }}{\equiv} T^{n+1} \cap G^{n+1}$ a free abelian subgroup of rank $n+1$ which generates over $R$ the vector space $T^{n+1}$ (i.e. $R^{n+1}=T^{n+1}$ ). Let $\sigma$ be the restriction to $G^{n+1}$ of the epimorphism $\sigma^{\prime}: E(n+1) \longrightarrow O(n+1)$ and put $K=$ Im $\sigma$. Then it follows from the definition that $U^{n+1}$ is normal in $G^{n+1}$, that $G^{n+1}$, $U^{n+1} \cong K \subset O(n+1)$ and that $K$ acts effectively on $U^{n+1}$. Furthermore $U^{n+1}$ is maximal abelian and of finite index in $G^{n+1}$. A set of points equivalent by the operation of $U^{n+1}$ is called a $n+1$-dimensional lattice $\Lambda$ (i.e. $U^{n+1} x_{0}=\Lambda$ ). $K$ is a point group leaving $\Lambda$ invariant.

Each space group appears in an extension

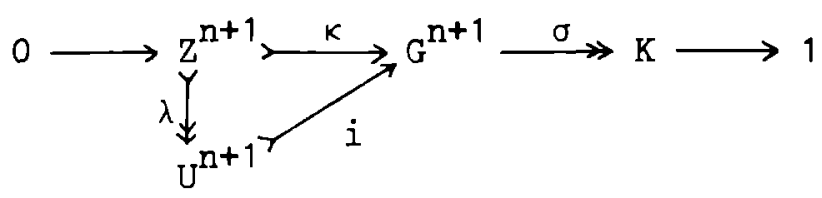

of $\mathrm{z}^{\mathrm{n}+1}$ by $\mathrm{K}$ with $\phi: \mathrm{K} \rightarrow \mathrm{GL}(\mathrm{n}+1, \mathrm{Z})$ a monomorphism. The subgroup $\mathrm{U}^{\mathrm{n}+1}$ is unique, so the monomorphism $k=i \circ \lambda$ is only variable in the isomorphism $\lambda$. Fixing $\lambda$ is equivalent to choosing a basis of $\Lambda$ or correspondingly a set of free generators of $U^{n+1}$. The monomorphism $\phi$ gives a $n+1$-dimensional integral faithful representation of $K$. The group $\phi(K)$ is called an arithmetic point group.

Consider next to the diagram (3.1) the commutative diagram

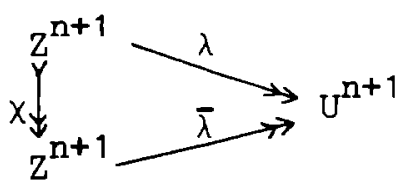

1) In diagrams we often use the following notation: a homomorphism is denoted by $\longrightarrow$, a monomorphism by $\longrightarrow$, an epimorphism by $\longrightarrow$, an isomorphism by $\longrightarrow$. 
where $\lambda$ inu $\bar{\lambda}$ are isomorphisms, $x$ an automorphism of $\mathrm{Z}^{\mathrm{n}+1}$, i.e. $x \in G L(n+1, Z)$. $\bar{\lambda}$ corresponds to another choice of basis $\mathrm{U}^{\mathrm{n}+1}$. Then (3.2) induces a morphism group extensions:

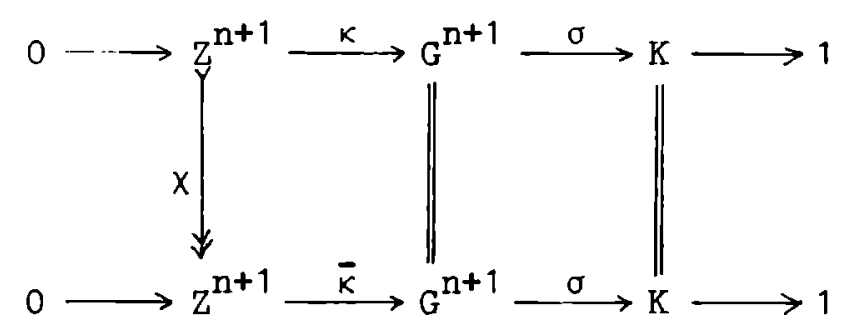

such that

$$
\bar{\phi}(\alpha)=x \phi(\alpha) x^{-1} \quad(\forall \alpha \in K)
$$

This means that $\phi$ and $\bar{\phi}$ are two Z-equivalent representations of $K$. The condition of arithmetic equivalence for two point groups $\phi(K)$ and $\bar{\phi}(K)$ is weaker:

$$
\bar{\phi}(K)=x \phi(K) x^{-1}
$$

and arises from the consideration of isomorphic space groups.

According to ref. [3], proposition 6, given two isomorphic space groups $G^{n+1}$ and $\bar{G}^{n+1}$, there exists a morphism of group extensions

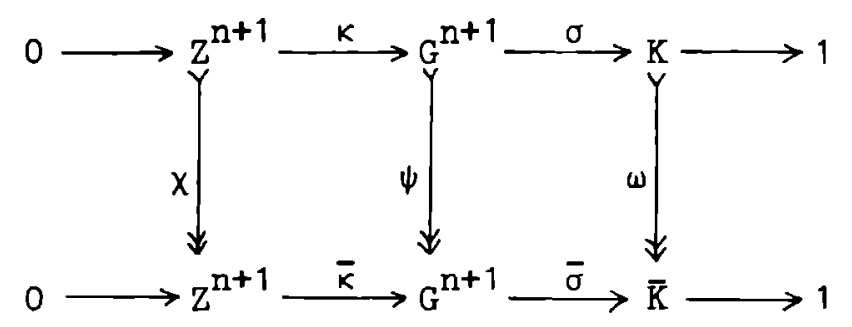

such that

$$
\bar{\phi}(\omega \alpha)=x \phi(\alpha) x^{-1} \quad(\forall \alpha \in K)
$$

with $\chi \in G L(n+1, z)$. This means that isomorphic space groups determine the same arithmetic class, i.e. a class of arithmetically equivalent point groups. As shown in ref. [3] in the lower extension one may always choose $\phi(K)=\bar{\phi}(\bar{K})$. 
We call $\phi(K)$ an arithmetic point group. Then denoting by $N_{\phi}(K)$ the normalizer of $\phi(K)$ in $G L(n+1, Z)$ :

$$
x \in N_{\phi(K)} \stackrel{\operatorname{def}}{\equiv}\left\{x \in G L(n+1, Z) \mid x \phi(K) x^{-1}=\phi(K)\right\},
$$

and one has the morphism

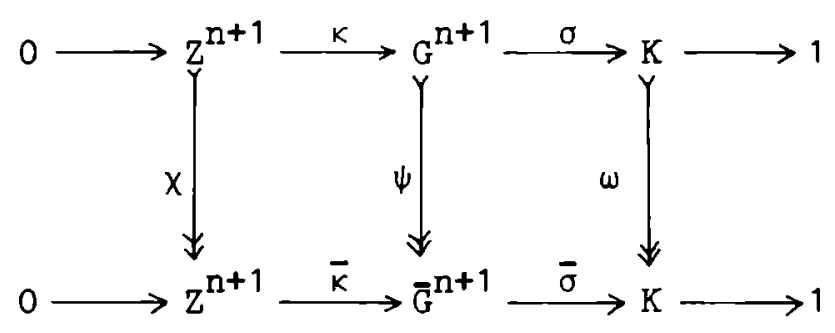

Hence one obtains all non-isomorphic space groups by finding all nonequivalent extensions of $\mathrm{z}^{\mathrm{n}+1}$ by one representative of each arithmetic crystal class.

The first problem is to find the arithmetic crystal classes. An element of a space group may be written as $(a, \alpha)$ with $a \in T^{n+1}$ and $\alpha \in O(n+1)$. For a given space group the elements $\alpha$ form the corresponding point group $K$. In $G L(n+1, R)$ the point groups of two affine equivalent space groups are conjugate by a non-singular matrix and therefore similar. But two similar point groups are even conjugate by an orthogonal transformation $[6, p .47]$. Therefore similar point groups are conjugate subgroups of $0(n+1)$, i.e. geometrically equivalent. The equivalence class is called geometric crystal class.

For a given point group $K$ one defined a subset $L_{K}$ of the set $L$ of $n+1-$ dimensional lattices $\Lambda$ as follows:

$$
\mathrm{L}_{\mathrm{K}}=\{\Lambda \in \mathrm{L} \mid \mathrm{K} \Lambda=\Lambda\} \text {. }
$$

Because of (3.1) and $(3.4)$ a pair $(K, \Lambda)$ with $\Lambda \in L_{K}$ defines and arithmetic crystal class.

\section{Proposition 5:}

If the point groups $K$ and $\bar{K}$ are geometrically equivalent, then for each $\Lambda \in \mathrm{L}_{\mathrm{K}}$ there is a $\Pi \in \mathrm{L}_{\overline{\mathrm{K}}}$ such that $(\mathrm{K}, \Lambda)$ and $(\mathbb{R}, \Lambda)$ determine the same 
arithmetic crystal class.

Proof:

$$
\begin{aligned}
& \overline{\mathrm{K}}=T \mathrm{~K} \mathrm{~T}^{-1} \text { for some } T \in O(n+1) \\
& \overline{\mathrm{K}}(\mathrm{T} \Lambda)=\mathrm{T} \Lambda \text {, so } \mathrm{T} \Lambda \in \mathrm{L}_{\overline{\mathrm{K}}} .
\end{aligned}
$$

On bases $B(\Lambda)$ of $\Lambda$ and $T B(\Lambda)$ of $T \Lambda$ both $K$ and $\bar{K}$ correspond to the same group of matrices $\phi(K)$.

\section{Consequence}

To obtain one representative from each arithmetic crystal class it is sufficient to consider one representative from each geometric class and to determine all arithmetic representations on bases of lattices left invariant by this representative.

The maximal point group leaving a lattice invariant is its holohedry $\mathrm{H}$. A lattice $\Lambda$ determines an arithmetic crystal class with representative $\phi(H)$, but the converse is not true. The arithmetic crystal class of the holohedry defines the Bravais class of $\Lambda$. Two lattices belong to the same Bravais class if and only if their arithmetic holohedries are arithmetically equivalent. In the same way the geometric class of the holohedry defines the system of $\Lambda$. Two lattices belong to the same system if and only if their holohedries are geometrically equivalent.

For given point group $K$ a point group $\bar{K}$ is called enveloping if for every $\Lambda \in I_{K}$ one has a group from the geometric class of $\bar{K}$ which leaves $\Lambda$ invariant. The maximal enveloping group of $K$ is called its system group $K_{0} \cdot$ The reason of this terminology is that, for $n \leqslant 3$, each system group $K_{0}$ is the holohedry of at least one lattice from $I_{K}$. So in these cases the system groups and geometric holohedries are the ${ }^{\mathrm{o}}$ same.

Example: 3-dimensional geometric groups, enveloping groups and system groups. Notation:

$A \rightarrow B: B$ is an enveloping group for $A$

C : C is a system group. 
Point group System group System

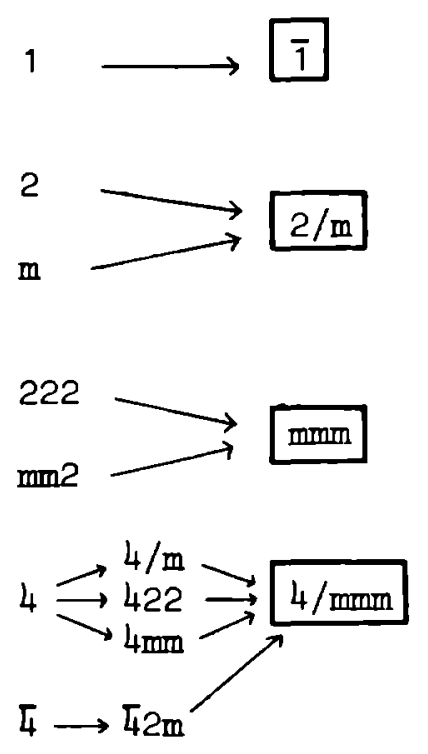

clinic)

(orthorhombic)

(tetragonal)
Point group System group System

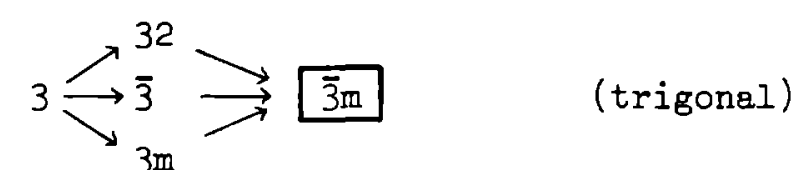

(hexagonal)

The set $I_{K_{O}}$ is divided in two subsets: to the first subset belong those lattices for which $K_{0}$ is the holohedry, to the second one those for which this is not the case. In both subsets, $\mathrm{L}_{\mathrm{K}_{\mathrm{O}}}^{\mathrm{I}}$ and $\mathrm{L}_{\mathrm{K}_{\mathrm{O}}}^{\mathrm{II}}$, an equivalence relation is defined:

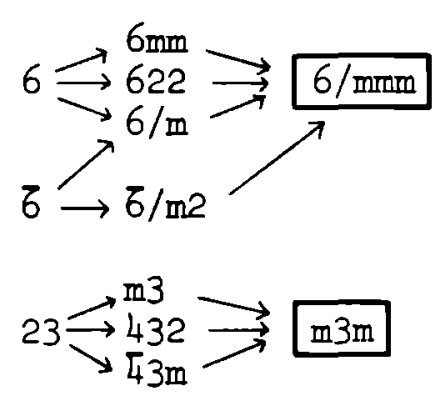

(cubic)

i) in $L_{K_{0}}^{I}$ two lattices $\Lambda_{1}$ and $\Lambda_{2}$ are equivalent if $\left(K_{0}, \Lambda_{1}\right)$ and $\left(K_{0}, \Lambda_{2}\right)$ determine the same arithmetic crystal class; these classes correspond to Bravais classes.

ii) in $L_{K_{0}}^{I I} \Lambda_{1}$ and $\Lambda_{2}$ are equivalent, if they belong to the same Bravais class.

The different arithmetic crystal classes corresponding to $K_{0}$ are found as follows. From each class in $\mathrm{L}_{\mathrm{K}_{0}}^{I}$ one chooses one representative $\Lambda$ and considers $\left(K_{0}, \Lambda\right)$; among the classes of $\mathrm{I}_{K_{0}}^{I I}$ one considers only those for which the holohedry $\phi\left(\mathrm{H}_{1}\right)$ has not a subgroup arithmetically equivalent to the holohedry $\phi\left(\mathrm{H}_{2}\right)$ of another class. From each of these classes one chooses one representative $\Lambda$ and considers $(H, \Lambda)$. The different arithmetic point groups $\phi\left(\mathrm{K}_{\mathrm{O}}\right)$ are arithmetically equivalent to a subgroup of a group from the class $(\mathrm{H}, \Lambda)$.

Example: The 3-dimensional system group $\mathrm{K}_{0}=\overline{3} \mathrm{~m}$ leaves invariant lattices of the trigonal, hexagonal and cubic systems. $\overline{3} m$ is holohedry for the rhombohedral lattices of the trigonal system. If we denote the arithmetic classes by the corresponding symorphic space group symbol, this defines the arith- 
metic point group $\mathrm{R} \overline{3} \mathrm{~m}$, which is an arithmetic holohedry. The arithmetic holohedries of the primitive, body-centered and face-centered cubic lattices have a subgroup arithmetically equivalent to $R \overline{3}$ m. So the arithmetic point groups corresponding to $\overline{3} m$ and not arithmetically equivalent to $R \overline{3} m$ are the arithmetically non-equivalent subgroups of an arithmetic holohedry of a hexagonal lattice. There are two of them, $P \overline{3} m 1$ and $P \overline{3} 1 \mathrm{~m}$. So all together there are three arithmetic crystal classes corresponding to $3 \mathrm{~m}$.

\section{Proposition 6:}

If $K_{O}$ is the system group of $K$, then each arithmetic group $\phi(K)$ is arithmetically equivalent to a subgroup of $\phi\left(K_{0}\right)$.

Proof: $\phi(K)$ is an integral faithful representation of $K$ related to a basis of a lattice $\Lambda$, such that $K \Lambda=\Lambda$. Then also $K_{0} \Lambda=\Lambda$. As $K \subseteq K_{0}$, also $\phi(K) \subseteq \phi\left(K_{0}\right)$

Note that $\phi\left(K_{0}\right)$ may contain several arithmetic point groups $\phi(K)$ which are arithmetically non-equivalent. As a consequence of proposition 6 it is sufficient to find the equivalence classes in $\mathrm{L}_{\mathrm{K}_{\mathrm{O}}}$ for each system group $\mathrm{K}_{\mathrm{O}}$ in order to obtain all arithmetically non-equivalent $\phi\left(\mathrm{K}_{\mathrm{o}}\right)$. Afterwards one determines for each subgroup $K \subset K_{0}$ arithmetically non-equivalent subgroups $\phi(K)$ of $\phi\left(K_{0}\right)$.

Finally one has to find all non-isomorphic extensions (3.1) for a given arithmetic point group $\phi(K)$. (For the theory of group extensions see ref. $[7],[8],[9]$ and [10]). Suppose the point group $K$ to be given by generators $\alpha_{1}, \ldots, \alpha_{v}$ and defining relations

$$
\phi_{i}\left(\alpha_{1}, \ldots, \alpha_{v}\right)=\varepsilon \quad(i=1, \ldots, r)
$$

and suppose that $r(\alpha)$ is a representative of the coset of $\mathrm{z}^{\mathrm{n}+1}$ in $\mathrm{G}^{\mathrm{n}+1}$ which is mapped by $\sigma(3.1)$ on $\alpha \in \mathrm{K}$. Then the words

$$
\kappa g_{i}=\phi_{i}\left(r\left(\alpha_{1}\right), \ldots, r\left(\alpha_{\nu}\right)\right) \quad(i=1, \ldots, r)
$$

belong to $\times \mathrm{z}^{\mathrm{n}+1}[6]$. In order to define a.group $\mathrm{G}^{\mathrm{n}+1}$ in the extension these $g_{i}$ have to be solutions of

$$
\sum_{i=1}^{r} h_{i} g_{i}=0
$$


where $H=\left[h_{1}, \ldots, h_{r}\right]$ are called the extension conditions for the group $K$ [6] , $[10]$. If $\left\{B_{i}\right\}_{i=1, \ldots, r}$ satisfy (3.6), the space group $G^{n+1}$ is generated by the free generators $k a_{1}, \ldots, k a_{n+1}$ of $k z^{n+1}$, the representatives $r\left(\alpha_{1}\right), \ldots, r\left(\alpha_{v}\right)$ and the relations

$$
\begin{cases}\kappa a_{i}+k a_{j}=k a_{j}+k a_{i} & (i, j=1, \ldots, n+1) \\ \phi_{i}\left(r\left(\alpha_{1}\right), \ldots, r\left(\alpha_{v}\right)=\kappa g_{i}\right. & (i=1, \ldots, r) \\ r(\alpha)+\kappa a-r(\alpha)=\kappa \alpha a \equiv \kappa \phi(\alpha) a & \left(a \in z^{n+1}, \alpha \in K\right)\end{cases}
$$

In the same way $\overline{\mathrm{G}}^{\mathrm{n}+1}$ is generated by $\bar{\kappa} a_{1}, \ldots, \bar{k} a_{n+1}$ and $\bar{r}\left(\alpha_{1}\right), \ldots, \bar{r}\left(\alpha_{v}\right)$. If an isomorphism $\psi: G^{n+1} \rightarrow \bar{G}^{n+1}$ exists such that $\psi r(\alpha)=\bar{k} c(\alpha)+\bar{r}(\alpha)(\forall \alpha \in K)$ for certain $c \in C_{\phi}^{l}\left(K, z^{n+1}\right)$, the extensions are equivalent. The equivalence classes of $(K, \phi)$-extensions of $z^{n+1}$ form an abelian group Ext $\left(K, z^{n+1}, \phi\right)$ [9] which is isomorphic to the second cohomology group $\mathrm{H}_{\phi}^{2}\left(\mathrm{~K}, \mathrm{z}^{\mathrm{n}+1}\right)$. Two groups $\mathrm{G}^{\mathrm{n}+1}$ and $\overline{\mathrm{G}}^{\mathrm{n}+1}$ appearing in equivalent extensions (3.5) are isomorphic. Ilowever also two groups belonging to non-equivalent extensions may be isomorphic. In this case they may be obtained from non-equivalent extensions with the same $\phi(K)$. According to [3], proposition 7, two groups in extensions with the same $\phi(K)$ are isomorphic if and only if it is possible to find representatives of their equivalence classes with factor systems $m$ and $\vec{m}$ respectively, an automorphism $\omega$ of $K$ and an element $x$ of the normalizer $N_{\phi(K)}$ of $\phi(K)$ in $\mathrm{GL}(n+1, z)$ such that

$$
\begin{array}{lc}
\overline{\mathrm{m}}(\omega \alpha, \omega \beta)=X \mathrm{~m}(\alpha, \beta) & (\forall \alpha, \beta \in \mathrm{K}) \\
\chi \phi(\alpha) \mathrm{a}=\phi(\omega \alpha) \times a & \left(\forall \alpha \in \mathrm{K}, \forall \mathrm{a} \in \mathrm{z}^{\mathrm{n}+1}\right) .
\end{array}
$$

Here we state another equivalent criterion applicable in the case the groups are given by generators and defining relations as in (3.7). If $\omega$ is an automorphism of $K$ choose for $\omega_{j}{ }_{j}$ a fixed word

$$
\omega \alpha_{j}=w_{j}\left(\alpha_{1}, \ldots, \alpha_{v}\right) \quad(j=1, \ldots, v)
$$




$$
\bar{\kappa} f_{i}\left(\bar{g}_{1}, \ldots, \bar{g}_{r}\right) \stackrel{\text { def }}{\equiv} \phi_{i}\left(w_{1}\left(\bar{r}\left(\alpha_{1}\right), \ldots, \bar{r}\left(\alpha_{\nu}\right)\right), \ldots, w_{v}\left(\bar{r}\left(\alpha_{1}\right), \ldots, \bar{r}\left(\alpha_{v}\right)\right)\right.
$$

where

$$
\bar{\kappa} \bar{g}_{i}=\phi_{i}\left(\bar{r}\left(\alpha_{l}\right), \ldots, \bar{r}\left(\alpha_{v}\right)\right) \quad(i=1, \ldots, r)
$$

Furthermore elements $\pi_{i}\left(\alpha_{j}\right)(i=1, \ldots, r ; j=1, \ldots, v)$ of the integral group ring $Z K$ are defined by

$$
\begin{aligned}
\phi_{i}\left(k c\left(\alpha_{1}\right)+r\left(\alpha_{1}\right), \ldots, k c\left(\alpha_{\nu}\right)\right. & \left.+r\left(\alpha_{v}\right)\right)=\kappa \sum_{j=1}^{\nu} \pi_{i}\left(\alpha_{j}\right) \cdot c\left(\alpha_{j}\right)+ \\
& +\phi_{i}\left(r\left(\alpha_{1}\right), \ldots, r\left(\alpha_{v}\right)\right) \quad(i=1, \ldots, r)
\end{aligned}
$$

\section{Proposition 7:}

$\mathrm{G}^{\mathrm{n}+1}$ and $\overline{\mathrm{G}}^{\mathrm{n}+1}$ are isomorphic if and only if there are a) an automorphism $\omega$ of $K, b)$ an element $x \in N_{\phi(K)}$ and $\left.c\right)$ elements $c(\alpha) \in z^{n+1}$ such that

$$
f_{i}\left(\bar{g}_{i}, \ldots, \bar{g}_{r}\right)+\sum_{j=1}^{v} \pi_{i}\left(w \alpha_{j}\right) \cdot c\left(\alpha_{j}\right)=x g_{i} \quad(i=1, \ldots, r)
$$

\section{Proof:}

a) If in (3.5) $\psi$ is an isomorphism, $x$ is the restriction of $\psi$ to $z^{n+1}$ and $\omega$ is the induced automorphism one has [3] :

$$
\phi(\omega \alpha)=x \phi(\alpha) x^{-1} \quad(\forall \alpha \in K)
$$

Therefore $x \in \mathbb{N}_{\phi(K)}$.

$\psi$ operating on the relation $\phi_{i}\left(r\left(\alpha_{1}\right), \ldots, r\left(\alpha_{\nu}\right)\right)=\kappa g_{i}$ gives

$$
\phi_{i}\left(\bar{r}\left(\omega \alpha_{1}\right), \ldots, \bar{r}\left(\omega \alpha_{v}\right)\right)+\bar{k} \sum_{j=1}^{v} \pi_{i}\left(\omega \alpha_{j}\right) \cdot c\left(\alpha_{j}\right)=\bar{k} \times g_{i} \quad(i=1, \ldots, r) .
$$

Choose for every $w \alpha \in K$ a fixed word $w_{\alpha}\left(\alpha_{1}, \ldots, \alpha_{v}\right)$.

Then $\bar{r}(\omega \alpha)=\bar{k} a(\omega \alpha)+w_{\alpha}\left(\bar{r}\left(\alpha_{1}\right), \ldots, \bar{r}\left(\alpha_{\nu}\right)\right)$ for some $a(\omega \alpha) \in z^{n+1}$. One may choose $a(w \alpha)=0$ for this choice of words, because this only gives an equivalent group. (Of course for another choice of words $w_{\alpha}^{\prime}\left(\alpha_{1}, \ldots, \alpha_{v}\right)$ in general $a(\omega \alpha) \neq 0$; it is well known that only in split extensions one can have repre- 
sentatives $r(\alpha)$ in such a way that $r(\alpha \beta)=r(\alpha)+r(\beta)$ for all $\alpha, \beta \in K)$. Doing this one obtains relation (3.8).

b) On the other hand, if $\omega$ and $x$ are given in such a way that $(3.8)$ is correct, one may define an isomorphism $\psi: G^{n+1}+\overline{\bar{G}}^{n+1}$, where $\bar{G}^{n+1}$ and $\overline{\bar{G}}^{n+1}$ appear in equivalent extensions, by

$$
\psi(k a+r(\alpha))=\bar{k} x a+\bar{k} c(\alpha)+\bar{r}(w \alpha) \quad \begin{aligned}
&( \forall a \in z^{n+1}, \\
& \forall \alpha \in K, \\
&\left.c(\alpha) \in \mathrm{z}^{n+1}\right)
\end{aligned}
$$

Then $\mathrm{G}^{\mathrm{n}+1}$ and $\overline{\mathrm{G}}^{\mathrm{n}+1}$ are isomorphic.

So from a set of non-equivalent extensions for a given $\phi(K)$ one can determine the non-isomorphic ones using proposition 7 .

\section{Relativistic crystallographic groups}

A relativistic space-time group $\mathrm{G}^{\mathrm{n}+1}$ is a subgroup of the inhomogeneous Lorentz group $J L(n+1)$ which contains a translation subgroup $U^{n+1} \stackrel{\operatorname{def}}{=} G^{n+1} n$ $T^{n+1}$ which is free abelian of rank $n+1$ and which over $R$ generates $T^{n+1}$. This group $U^{n+1}$ is maximal abelian and normal in $\mathrm{G}^{\mathrm{n}+1}$. Analogously to the Euclidean case the relativistic point group $K \cong G^{n+1} / U^{n+1}$ is a subgroup of $0(n, 1)$ leaving $\Lambda$ invariant and $G^{n+1}$ appears in an extension

$$
0 \longrightarrow \mathrm{z}^{\mathrm{n}+1} \longrightarrow \mathrm{G}^{\mathrm{n}+1} \longrightarrow \mathrm{K} \longrightarrow 1
$$

of a free abelian group $\mathrm{z}^{\mathrm{n}+1}$ of rank $\mathrm{n}+1$ by the point group $K$, $\phi$ being a monomorphism $K \rightarrow G L(n+1, Z)$. One of the most important differences from the Euclidean case is the fact that in general $K$ is not finite. In the present paper however we are concerned with space-time groups for which the image in $\operatorname{GIL}(n+1, R)$ coincides with the image of a Euclidean space group. Therefore the point groups considered here are always finite.

In Minkowskian space timelike, spacelike and isotropic translations occur (having negative, positive and zero norm respectively). For that reason we need a stronger equivalence relation than plain group isomorphism in order to decide if two relativistic space-time groups may be identified or not. 
Hence we define: two space-time groups $G^{n+1}$ and $\bar{G}^{n+1}$ are isomorphic if there is a group isomorphism $\psi: G^{n+1} \rightarrow \bar{G}^{n+1}$ such that for the restriction $x_{0}=\left.\psi\right|_{U^{n+1}}$
one has

$$
\operatorname{sign}\left\|x_{0} t\right\|^{2}=\operatorname{sign}\|t\|^{2} \quad\left(\forall t \in U^{n+1}\right)
$$

Here the sign of the norm of an isotropic vector is zero by definition.

Consider a space-time group $\mathrm{G}^{\mathrm{n}+1}$ (If not stated otherwise in this section we mean by space-time group always a relativistic space-time group), and the commutative diagram

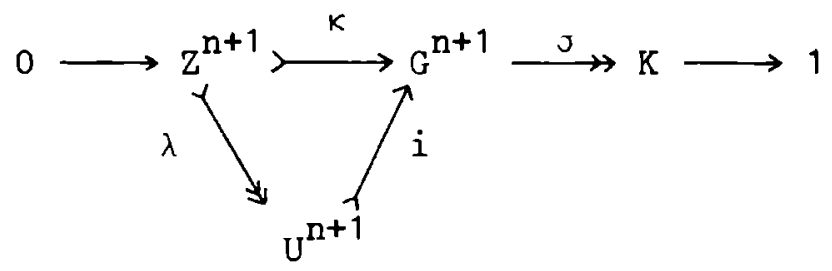

Here $i$ is the injection of the normal subgroup $U^{n+1}$ into $G^{n+1} \cdot \lambda$ is an isomorphism which determines a set of generators of $U^{n+1}$. If $\varepsilon_{1}=(1,0, \ldots, 0)$, $\varepsilon_{2}=(0,1, \ldots, 0), \ldots, \varepsilon_{n+1}=(0, \ldots, 0,1)$, then a basis $B=\left(a_{1}, a_{2}, \ldots, a_{n+1}\right)$ of the lattice $\Lambda$ is defined by $\lambda \varepsilon_{i}=a_{i}$. For the monomorphism $k$ one has $\kappa=i \circ \lambda$. The reason why we consider $i$ and $\lambda$ next to $\kappa$ is the fact that space-time groups are not uniquely determined by their abstract group structure. To the basis $B$ corresponds a metric tensor $g=g(B)$ with elements

$$
g_{i j}=a_{i} \cdot a_{j} \quad(i, j=1, \ldots, n+1) .
$$

The scalar product is given by the indefinite metric of $\mathrm{U}^{\mathrm{n}+1}$ induced by that of $\mathrm{T}^{\mathrm{n}+1}$. A discrete translation group $\mathrm{U}^{\mathrm{n}+1}$ (or the lattice $\Lambda$ obtained in Minkowskian space by operating with $\mathrm{U}^{\mathrm{n}+1}$ on the origin) is defined by its metric tensor up to a homogeneous Lorentz transformation. As $\mathrm{K}$ is a group of homogeneous Lorentz transformations leaving invariant the metric tensor one has

$$
g(B)=\phi^{t}(\alpha) g(B) \phi(\alpha) \quad(\text { any } \alpha \in K)
$$

where $g(B)$ and $\phi(\alpha)$ refer to the same lattice basis $B$. 
Now consider two extensions in which the same space-time group $G^{n+1}$ occurs :

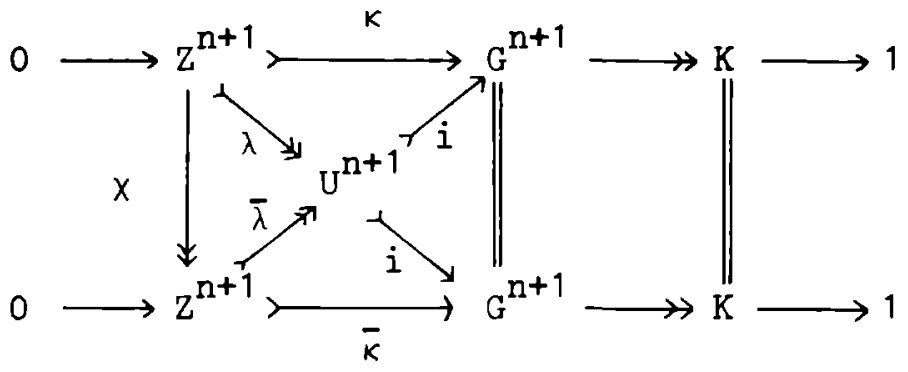

The elements $\bar{a}_{i}=\bar{\lambda} \varepsilon_{i}(i=1,2, \ldots, n+1)$ form another basis $\bar{B}$ of $\Lambda$. The monomorphism $\bar{\phi}: K \rightarrow G L(n+1, Z)$ is given by

$$
\bar{\phi}(\alpha)=x \phi(\alpha) x^{-1} \quad(\forall \alpha \in K)
$$

The metric tensor corresponding to the basis $\bar{B}=\left(\bar{a}_{1}, \bar{a}_{2}, \ldots, \bar{a}_{n+1}\right)$ is given by:

$$
g(B)=x^{t} g(\bar{B}) x
$$

So a space-time group defines a class of pairs $(B, \phi(K))$ with the following equivalence relation: two pairs $(B, \phi(K))$ and $(\bar{B}, \bar{\phi}(K))$ are equivalent if:

$$
\bar{\phi}(\alpha)=x \phi(\alpha) x^{-1} \quad(\forall a \in K)
$$

and

$$
g(B)=x^{t} g(\bar{B}) x
$$

Now consider two isomorphic space-time groups $G^{n+1}$ and $\bar{G}^{n+1}$ in the commutative diagram

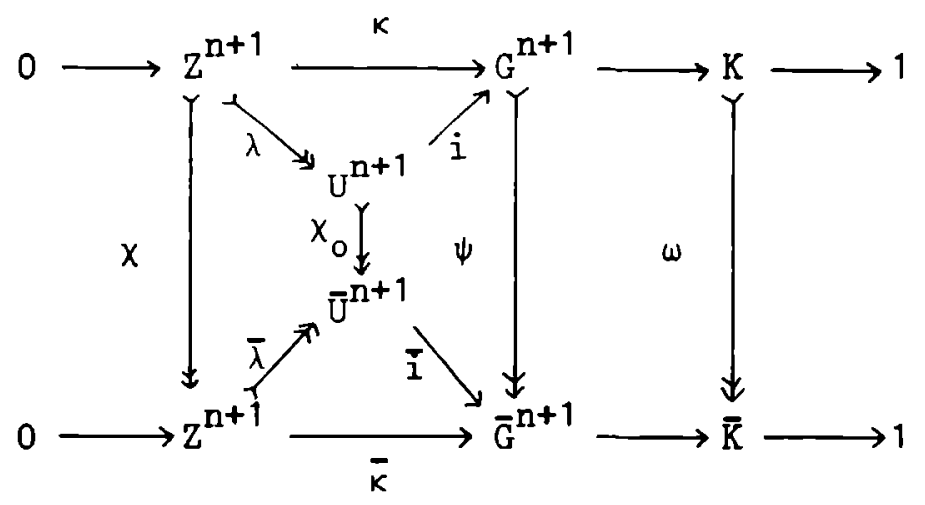


then $\psi$ is an isomorphism such that the restriction $x_{0}$ to $U^{n+1}$ satisfies relation (4.1). i and $\bar{i}$ are the natural injections, so $K \cong G^{n+1} / U^{n+1} \cong \bar{G} / \bar{U}^{n+1} \cong \bar{K}$. For the arithmetic point groups one has the relation

$$
\bar{\phi}(\omega \alpha)=x \phi(\alpha) x^{-1} \quad(\forall \alpha \in K)
$$

Now consider another basis $B^{\prime}$ of $\bar{\Lambda}$ determined by an isomorphism $\lambda^{\prime}=\bar{\lambda} x$ : $\mathrm{z}^{\mathrm{n}+1} \rightarrow \overline{\mathrm{U}}^{\mathrm{n}+1}$. Then we may insert a third exact row in (4.4) as follows

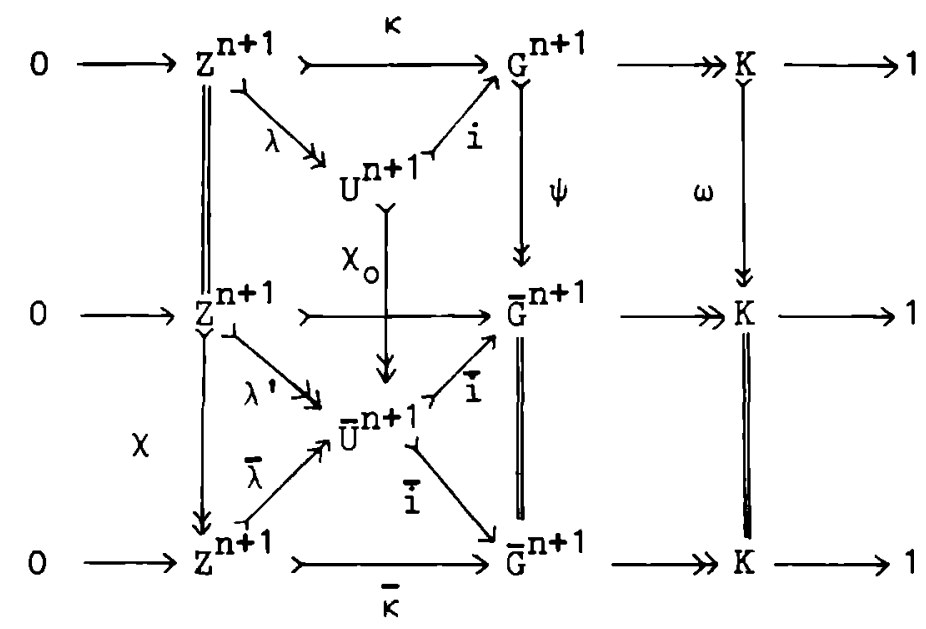

The arithmetic point groups are related by

$$
\bar{\phi}(\omega \alpha)=x \phi^{\prime}(\omega \alpha) x^{-1}=x \phi(\alpha) x^{-1}
$$

So

$$
\phi^{\prime}(\omega \alpha)=\phi(\alpha) \text {. }
$$

Hence the isomorphic space-time groups $G^{n+1}$ and $\bar{G}^{n+1}$ may be obtained in $(K, \phi)$ extensions with the same $\phi$.

If the metric tensor for the basis $B$ of $\Lambda$ is $B$ and that for $B^{\prime}$ of $\bar{\Lambda}$ is $g^{\prime}$ (4.1) requires

$$
\begin{aligned}
\sum_{i, j=1}^{n+1} p^{i} p^{j} g_{i j} \gtreqless 0 \text { if and only if } \sum_{i, j=1}^{n+1} p^{i} p^{j} g_{i j}^{j} \gtreqless 0 \\
\left(\text { any } p^{i} \in Z\right) .
\end{aligned}
$$

Suppose $g_{k k} \neq 0$ for all $1 \leqslant k \leqslant n+1$. This hypothesis is not restrictive because one can always find a basis without isotropic vectors. Then the images 
in $\bar{U}^{n+1}$ also have non-zero norm. One may suppose $p^{n+1} \neq 0$. By a change of variables

$$
q^{i}=\frac{p^{i}}{p^{n+1}} \quad(i=1, \ldots, n)
$$

(4.6) becomes

$$
f\left(q^{1}, \ldots, q^{n}\right) \stackrel{\text { def }}{=} \sum_{i, j=1}^{n} q^{i} q^{j} g_{i j}+\sum_{i=1}^{n} q^{i} g_{i(n+1)}+g_{(n+1)(n+1)} \gtreqless 0
$$

if and only if:

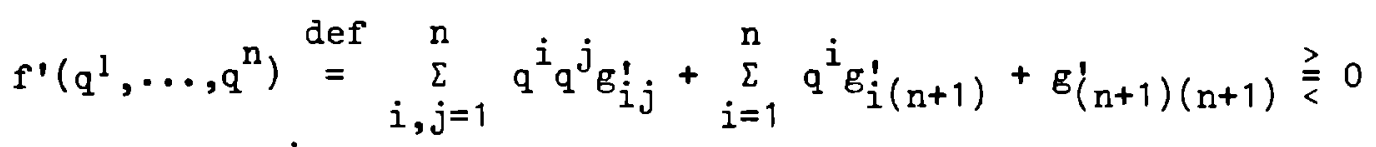

for all rational $q^{i}$.

Because the quadratic forms $f$ and $f^{\prime}$ are zero for the same values of the rational variables they only differ by a real factor $k$. As the regions of the variables, where the forms are positive, are the same, this $\mathrm{k}$ is positive.

So the equivalent space-time groups $G^{n+1}$ and $\bar{G}^{n+1}$ determine together with the isomorphisms $\lambda$ and $\bar{\lambda}$ two pairs $(B, \phi(K))$ and $(\bar{B}, \bar{\phi}(K))$ respectively such that

$$
\begin{aligned}
& g(B)=k x^{t} g(\bar{B}) x \\
& \bar{\phi}(K)=x \phi(K) x^{-1} \quad x \in G L(n+1, z), k>0
\end{aligned}
$$

Any two pairs which satisfy the relations (4.7) are called equivalent. The equivalence class is called the relativistic arithmetic crystal class $\{\mathrm{B}, \phi(\mathrm{K})\}$.

From the discussion above and the fact that isomorphisms $\lambda: z^{n+1} \rightarrow U^{n+1}$ and $\bar{\lambda}: \mathrm{z}^{\mathrm{n}+1} \rightarrow \overline{\mathrm{U}}^{\mathrm{n}+1}$ determine the same arithmetic crystal class according to (4.3) one has:

\section{Proposition 8:}

A space-time group determines a relativistic arithmetic crystal class. Isomorphic space-time groups determine the same relativistic arithmetic crystal class.

To find the arithmetic crystal classes one introduces the concept of geometric 
crystal class. Two point groups are called relativistic geometrically equivalent if they are conjugate subgroups of $0(n, 1)$.

Proposition 9:

If $K$ and $\bar{K}$ are relativistic geometrically equivalent point groups, for each space-time group with point group $\bar{K}$ one has an isomorphic space-time group with point group $\mathrm{K}$.

Proof: Suppose $K=T \bar{K} T^{-1}$ for some $T \in O(n, 1)$. The space-time group $\bar{G}^{n+1}$ occurs in a $(\bar{K}, \phi)$-extension, where $\phi(\bar{K})$ is $\bar{K}$ with respect to a basis $\bar{B}$. Then there is an isomorphic $(K, \phi)$-extension for $\phi(K)$ given by $K$ with respect to $\mathrm{B}=\mathrm{T} \overline{\mathrm{B}}$. (Note that $\phi(\mathrm{K})=\phi(\overline{\mathrm{K}})$ ). Because a Lorentz transformation leaves invariant the metric tensor, the translation groups $U^{n+1} \subset G^{n+1}$ and $\bar{U}^{n+1} \subset \bar{G}^{n+1}$ have the same metric tensor with respect to bases $B$ and $\bar{B}$ respectively. Hence $G^{n+1}$ and $\bar{G}^{n+1}$ are relativistic equivalent.

Proposition 10:

It is sufficient to consider the relativistic arithmetic crystal classes for one representative of each relativistic geometric class in order to obtain all relativistic arithmetic crystal classes.

Two lattices $\Lambda$ and $\bar{\Lambda}$ generated by the translation subgroups $U^{n+1} \subset G^{n+1}$ and $\bar{U}^{n+1} \subset \bar{G}^{n+1}$ of two isomorphic space-time groups have bases with respect to which the metric tensors differ only by a positive real factor. This means that both lattices have the same holohedry $\phi(H)$ with respect to these bases. Therefore the concept of Bravais class does not play the same important role here as it does in the Euclidean case.

Two lattices $\Lambda$ and $\bar{\Lambda}$ belong to the same relativistic Bravais class if there exists an isomorphism between the generating translation groups $U^{n+1}$ and $\bar{U}^{n+1}$ that maps elements of $U^{n+1}$ on elements of the same kind in $\bar{U}^{n+1}$. (of course both are isomorphic to $\mathrm{z}^{\mathrm{n}+1}$ ). From the foregoing discussion it follows that $\Lambda$ and $\bar{\Lambda}$ belong to the same relativistic Bravais class if there are bases $B$ of $\Lambda$ and $\bar{B}$ of $\bar{\Lambda}$ such that for the corresponding metric tensors one has

$$
\mathrm{g}(\mathrm{B})=\mathrm{kg}(\overline{\mathrm{B}}) \quad \mathrm{k}>0
$$

Hence a Bravais class may be denoted by a $g(B)$ or by a class $\{B, \phi(H)\}$ because $B$ determines the holohedry $\phi(H)$. Each arithmetic crystal class 
$\{B, \phi(K)\}$ belongs to a Bravais class $\{B, \phi(H)\}$ and one has $\phi(K) \subseteq \phi(H)$.

In the same way as in the Euclidean case one defines a relativistic system. Two lattices belong to the same relativistic system if their geometric holohedries are geometrically equivalent.

Finally one has to find all non-isomorphic space-time groups obtained from a $(K, \phi)$-extension and with basis $B$ for one representative of each arithmetic crystal class $\{B, \phi(K)\}$.

Consider two isomorphic space-time groups $G^{n+1}$ and $\bar{G}^{n+1}$. They can always be made to appear in the following commutative diagram

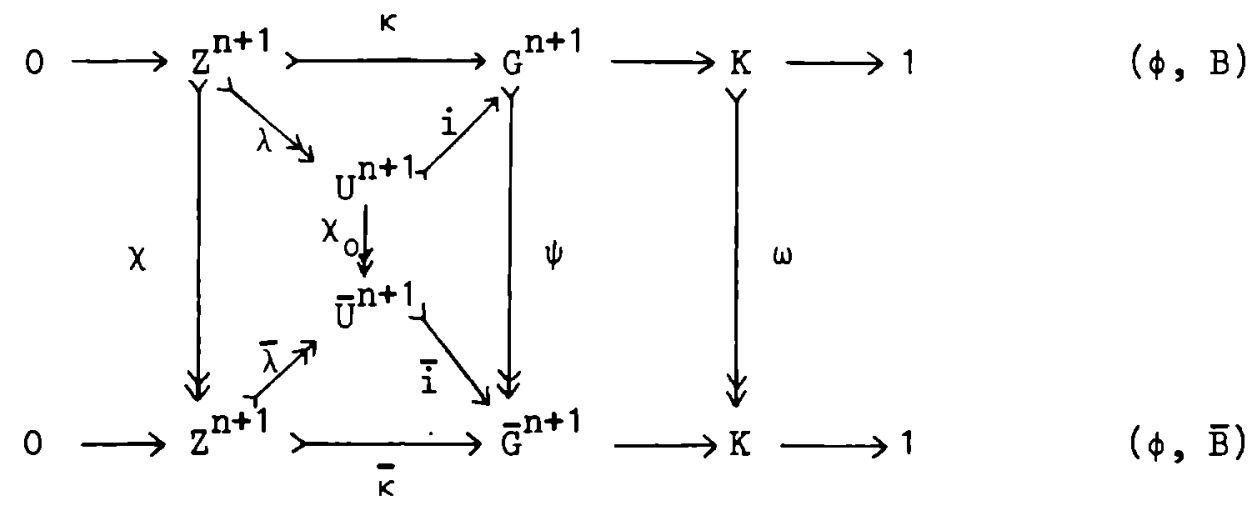

where $\mathrm{kg}(\overline{\mathrm{B}})=\mathrm{g}(\mathrm{B})$.

From $g(B)=k x^{t} g(\bar{B}) x$ one has $x \in \phi(H)$.

Furthermore because $\phi(\omega \alpha)=\chi \phi(\alpha) x^{-1}$ one also has $\chi \in N_{\phi}$. Therefore $x \in \mathbb{N}_{\phi} \cap \phi(H)$.

Proposition 11:

If two space-time groups $G^{n+1}$ and $\bar{G}^{n+1}$ appear in equivalent extensions (4.8) they are isomorphic.

Proof: If the upper and lower rows in (4.8) are equivalent one may choose the isomorphism $\psi$ in such a way that $x$ is the identity mapping. This means

$$
g(B)=k g(\bar{B})
$$

So $\mathrm{G}^{\mathrm{n}+1}$ and $\overline{\mathrm{G}}^{\mathrm{n}+1}$ are isomorphic.

As a consequence of propositions 7 and 11 one may formulate the following criterion to distinguish the non-isomorphic space-time groups appearing in (4.8). (We use here the same notation as in section 3 ).

Proposition 12:

$\mathrm{G}^{\mathrm{n+1}}$ and $\overline{\mathrm{G}}^{\mathrm{n}+1}$ are isomorphic space-time groups if and only if there are auto- 
morphisms $\omega \in$ Aut $K$ and $x \in \mathbb{N}_{\phi(K)} \cap \phi(H)$ and elements $c\left(\alpha_{1}\right), \ldots, c\left(\alpha_{\nu}\right) \in z^{n+1}$ such that relation (3.8) is satisfied, i.e.:

$$
f_{i}\left(\bar{g}_{1}, \ldots, \bar{g}_{r}\right)+\sum_{j=1}^{\nu} \pi_{i}\left(\omega \alpha_{j}\right) c\left(\alpha_{j}\right)=x g_{i} \quad(i=1, \ldots, r)
$$

Proof: As a consequence of proposition 7 : if $\mathrm{G}^{\mathrm{n}+1}$ and $\overline{\mathrm{G}}^{\mathrm{n}+1}$ have isomorphic ab. iract structure, one has $x \in \mathbb{N}_{\phi(K)}$. Besides, as seen above, $x \in \phi(H)$.

On the other hand if $x \in \mathbb{N}_{\phi}(K) \cap \phi(H)$ the groups $G^{n+1}$ and $\bar{G}^{n+1}$ considered in the proof of proposition 7 are not only isomorphic as abstract groups, but even as relativistic space-time groups (Groups obtained from equivalent extensions are isomorphic).

\section{Galilean crystallographic groups}

A Galilean space-time group $\mathrm{G}^{\mathrm{n}+1}$ is a subgroup of $\mathrm{JG}(\mathrm{n}+1)$ with a translation subgroup $U^{n+1}=G^{n+1} n T^{n+1}$ which is free abelian of rank $n+1$ and which over $R$ generates $T^{n+1}$. A Galilean point group $K$ is a subgroup of $G(n+1)$ which leaves a $n+1$-dimensional lattice invariant. As we are only concerned with Galilean space-time groups for which the image in $G I L(n+1, R)$ coincides with the image of a Euclidean space group, these Galilean space-time groups are affine conjugate if and only if they have isomorphic group structure. However, in Galilean space one can distinguish two types of vectors: spacetype $\left(x^{n+1}=0\right)$ and velocity-type $\left(x^{n+1} \neq 0\right)$. Therefore we call two Galilean space-time groups isomorphic if there is a group isomorphism between them such that all translation elements of one group are mapped on translation elements of the same type of the other one.

Denoting by $\Lambda$ the lattice generated by $U^{n+1}$ from a given origin and by $\underline{R}^{\mathrm{n}}$ the hyperplane $x^{\mathrm{n}+1}=0$, we define

$$
\lambda \stackrel{\operatorname{def}}{=} \Lambda \cap \underline{R}^{\mathrm{n}} .
$$

\section{Proposition 13:}

$\lambda$ is a $\ell$-cimensional lattice for certain $\ell(0 \leqslant \ell \leqslant n)$. This is denoted by $\lambda_{\ell}$. 


\section{Proof:}

i) $\lambda_{\ell}$ is discrete, $\Lambda$ being a lattice;

ii) if $r_{1}, r_{2} \in \lambda_{\ell}$, then $n_{1} r_{1}+n_{2} r_{2} \in \lambda_{\ell}$ for any $n_{1}, n_{2} \in Z$; consequently $\lambda_{\ell}$ is a module over $\mathrm{Z}$;

iii) $\lambda_{\ell}$ is generated by at most $\mathrm{n}$ basis vectors (which can be chosen as basis vectors of $\Lambda$ ).

It is always possible to choose a basis $a_{1}, \ldots, a_{n+1}$ of $\Lambda$ in such a way that $a_{1}, \ldots, a_{\ell}$ are basis vectors of $\lambda_{\ell}$. This is called a standard basis of $\Lambda$. If, for one choice of a standard basis, $G^{n+1}$ determines the arithmetic point group $\phi(K)$, then for another choice one has

$$
\bar{\phi}(\alpha)=x \phi(\alpha) x^{-1} \quad(\forall \alpha \in K)
$$

where

$$
x=\left(\begin{array}{ll}
x_{1} & x_{2} \\
0 & x_{3}
\end{array}\right)
$$

and $x \in G L(n+1, z), x_{1} \in G L(l, z), x_{3} \in G L(n+1-l, z)$. So a Galilean space-time group determines on a standard basis the dimension $\ell$ of $\underline{R}^{n} \cap \Lambda$ and $a \phi(K)$ up to conjugation by an element $x(5.2)$.

Two pairs $[\bar{\phi}(K), \bar{l}]$ and $[\phi(K), \ell]$ are equivalent if

$$
\text { i) } \ell=\bar{\ell}
$$

ii) an element $x \in G L(n+1, z)$ of the form (5.2) exists, such that (5.1) is valid.

\section{A Galilean arithmetic crystal class is an equivalence class of pairs} $[\phi(K), \ell]$.

Then two isomorphic Galilean space-time groups determine the same Galilean arithmetic crystal class. On the other hand, if $[\phi(K), \ell]$ and $[\bar{\phi}(K), \bar{l}]$ are in the same Galilean arithmetic crystal class, for each Galilean spacetime group $G^{n+1}$ in a $(K, \phi)$-extension, there is an isomorphic one $\bar{G}^{n+1}$ in a $(K, \bar{\phi})$-extension. 
Two point groups are in the same Galilean geometric crystal class if they are conjugate subgroups of $G(n+1)$. Again (cf. proposition 10) to obtain all Galilean arithmetic crystal classes it is sufficient to consider one representative $K$ of each geometric crystal class. For, suppose $K=T \bar{K} T^{-1}$ for some $T \in G(n+1)$ and let $\bar{K} \bar{\Lambda}=\bar{\Lambda}$, then $K T \bar{\Lambda}=T \bar{\Lambda} \stackrel{\text { def }}{=} \Lambda$. If $\bar{B}$ is a standard basis of $\bar{\Lambda}$, and $\operatorname{dim}\left(\underline{R}^{n} \cap \bar{\Lambda}\right)=\ell$, then $B=T \bar{B}$ is a standard basis of $\Lambda$ and $\operatorname{dim}\left(\underline{R}^{n} \cap \Lambda\right)=\ell$, because $G(n+1) \cdot \underline{R}^{n}=\underline{R}^{n}$.

A lattice $\Lambda$ determines an arithmetic holohedry, i.e. the Galilean arithmetic crystal class of the pair $[\phi(H), l]$, when $H$ is the maximal point group leaving $\Lambda$ invariant. Two lattices belong to the same Galilean Bravais class if and only if they determine the same arithmetic holohedry.

Definition: a split lattice is a $n+1$-dimensional lattice for which a standard basis may be chosen with $\ell=n$ and with $a_{n+1}$ along the $x^{n+1}$-axis. Proposition 14:

In each Galilean Bravais class for which $\ell=\operatorname{dim}\left(\underline{R}^{n} \cap \Lambda\right)=n$ there is a split lattice.

Proof: As $\ell=n$ one has for $\Lambda$ a standard besis with $a_{1}, \ldots, a_{n} \in R^{n}$ and $a_{n+1}=\left(r_{n+1}, t_{n+1}\right)$. Define

$$
r(v)=\left(\begin{array}{rr}
\mathbb{1}_{n} & -v \\
0 . .0 & 1
\end{array}\right)
$$

where $r_{n+1}$ is the column vector with components $x_{n+1}^{1}, \ldots, x_{n+1}^{n}$ and $v=r_{n+1} t_{n+1}$. def

Then $\bar{\Lambda}=\gamma(v) \Lambda$ is a split lattice, because $\bar{\Lambda}$ admits the standard basis:

$$
\begin{aligned}
& \bar{a}_{i}=a_{i} \in \underline{R}^{n} \quad(i=1, \ldots, n) \\
& \bar{a}_{n+1}=\left[0, t_{n+1}\right] .
\end{aligned}
$$

$\Lambda$ and $\bar{\Lambda}$ being obtained from each other by a homogeneous Galilean transformation, they belong to the same Galilean Bravais class.

We have restricted ourselves here to space-time groups with a finite point group. However in the general case one may state the following propositions. 


\section{Proposition 15:}

The holohedry of a Galilean lattice $\Lambda$ with $\ell=n$ contains a free abelian subgroup of rank $\mathrm{n}$.

Proof: Because of proposition 14 one may consider a split lattice in the same Bravais class. Denote the Galilean geometric holohedry by $\mathrm{H}$ and suppose

$$
r(v)=\left(\begin{array}{cc}
R & v \\
0 & 1
\end{array}\right) \in H
$$

Then in $\underline{R}^{\mathrm{n}}$ one has: $\mathrm{R} \lambda_{\ell}=\lambda_{\ell}$. So, denoting the Euclidean holohedry of $\lambda_{\ell}$ by $h$, one has $R \in h$. In the hyperplane $x^{n+1}=t_{n+1}$ one has

$$
\gamma(v)\left[a_{n+1}+\lambda_{\ell}\right]=a_{n+1}+\lambda_{\ell},
$$

as these are exactly the points in this hyperplane which is left invariant by a Galilean transformation. Hence $v t_{n+1} \in \lambda_{l}$. So $H$ has the following elements

i) $\left(\begin{array}{ll}R & 0 \\ 0 & 1\end{array}\right) \quad(\forall R \in h)$

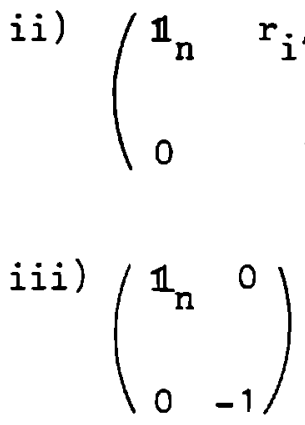

The elements $i i)$ are of infinite order and generate a free abelian group of rank $n$.

Proposition 16:

The holohedry $\mathrm{H}$ of a Galilean lattice $\Lambda$ with $\ell<\mathrm{n}$ has only elements of finite order. 
Proof: Let $a_{1}, \ldots, a_{n+1}$ with $a_{i}=\left[r_{i}, t_{i}\right]$ and $r_{i} \in \underline{R}^{n}(i=1, \ldots, n)$ be a standard basis of $\Lambda$. Over $z$ the vectors $a_{1}, \ldots, a_{l}$ generate $\lambda_{l}=\Lambda \cap \underline{R}^{n}$, over $F$ they generate the vector space $R^{l}$. Choose an orthonormal basis such that $e_{1}, \ldots, e_{\ell}$ generate $R^{l}$ and $e_{1}, \ldots, e_{n}$ generate $\underline{R}^{n}$, whereas $e_{n+1}$ is along the $\mathrm{x}^{\mathrm{n}+1}$-axis. With respect to this basis an element of the holohedry $\mathrm{H}$ has the form

$$
\gamma=\left(\begin{array}{ll}
P & u \\
& \\
0 & \varepsilon
\end{array}\right)
$$

where $P \in O(n)$, such that $P \lambda_{l}=\lambda_{\ell}$, $u$ is a $n$-dimensional column vector and $\varepsilon= \pm 1$. Suppose $\varepsilon=+1$. Consider the hyperplane $t=t_{i}(i=\ell+1, \ldots, n+1)$. In this hyperplane $\gamma$ operates as an element $\left(P, u t_{i}\right)$ of $E(n)$ and $\left(P, u t_{i}\right)$ leaves invariant the point sets $r_{i}+\lambda_{\ell}$ and $r_{i}+R^{l}$. Either $r_{i} \in R^{l}$ or $r_{i} \notin R^{l}$. i) If $r_{i} \in R^{l}$ define $v_{i}$ in the orthoplement $R_{\perp}^{n-l}$ of $R^{l}$ in $\underline{R}^{n}$ in such a way that $v_{i}+R^{\ell}=r_{i}+R^{l}$. Then $\left(P, u t_{i}\right)$ leaves invariant the set $r_{i}+\lambda_{\ell}$ in the hyperplane $v_{i}+R^{\ell}$. So $u \in R^{\ell}$ and $P v_{i}=v_{i}$. ii) If $r_{i} \in R^{l}$, one has $u \in R^{l}$ and $v_{i}=0$. So in $R_{\perp}^{n-\ell}$ the vectors $v_{\ell+1}, \ldots, v_{n+1}$ are left invariant by $P$. These vectors span a d-dimensional space and $d=n-l$, because, if $d<n-l$ the basis vectors $a_{1}, \ldots, a_{n+1}$ would generate a space of dimension $\leqslant \ell+d+1<n+1$. Therefore $P$ leaves $R_{\perp}^{n-\ell}$ pointwise fixed. So $\gamma$ has the form,

$$
\gamma=\left(\begin{array}{ccc}
P_{\ell} & 0 & u_{\ell} \\
0 & \mathbb{1}_{n-\ell} & 0 \\
0 & 0 & 1
\end{array}\right)
$$

where $P_{\ell} \lambda_{\ell}=\lambda_{\ell}$ and $u_{\ell}$ a $\ell$-dimensional column vector. $P_{\ell}$, being an element of a $\ell$-dimensional Euclidean point group, is of finite order m. Hence

$$
\gamma^{m}=\left(\begin{array}{cc} 
& w_{\ell} \\
\mathbb{1}_{n} & 0 \\
0 & 1
\end{array}\right)
$$


$\gamma^{\mathrm{m}}$ leaves invariant $a_{\ell+1}+\lambda_{\ell}$. So $t_{\ell+1} w_{\ell} \in \lambda_{\ell}$. In the same way $t_{\ell+2} w_{\ell} \in \lambda_{\ell}$ : Suppose $w_{\ell} \neq 0$. Then $t_{\ell+1} / t_{\ell+2}=p / q$ is a rational number $(p, q \in Z)$ and $\mathrm{q}_{\ell+1}-\mathrm{p} \mathrm{t}_{\ell+2}=0$, i.e.

$$
q a_{\ell+1}-p a_{\ell+2} \in \underline{R}^{n} \cap \Lambda=\lambda_{\ell} \text {. }
$$

Ey hypothesis this not being the case, $w_{\ell}=0$ and therefore $\gamma$ is of finite order. Because the elements with $E=+1$ form a subgroup of index 1 or 2 in the holohedry the proposition has been shown.

To obtain the Galilean arithmetic crystal classes, one takes one representative $K$ of each Galilean geometric class and determines the lattices left invariant by $K$. One takes one representative $[\phi(H), l]$ of each arithmetic crystal class of the holohedries of these lattices. (We remark that if a lattice occurs with $\ell=m$, there exist also lattices with $\ell=m+1, \ldots, n)$. The non-equivalent pairs $[\phi(K), l]$ with $K \subseteq \mathrm{H}$ are representatives of the Galilean arithmetic crystal classes.

Now still remains the problem of finding all non-isomorphic Galilean space-time groups for a given $[\phi(K), \ell]$. Consider the morphism of group extensions:

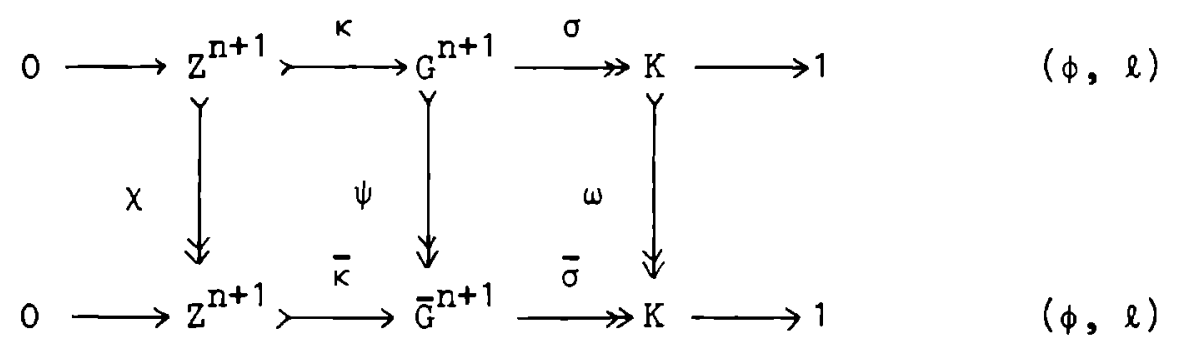

and the commutative diagram

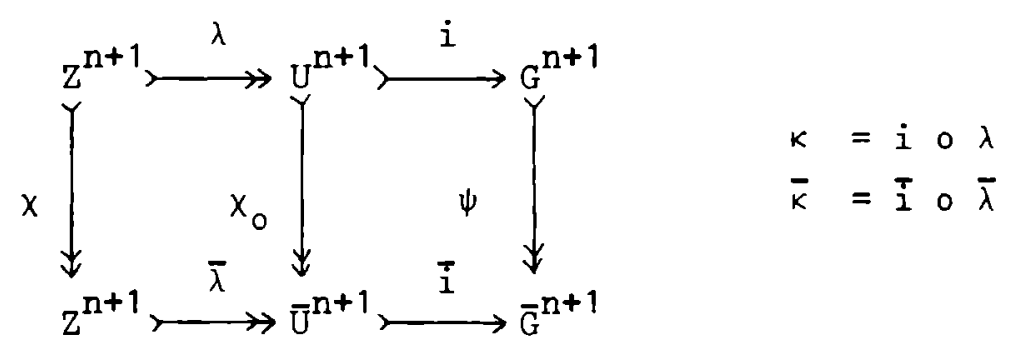

If both extensions (5.4) are equivalent, $x$ can be chosen to be the identity. So $\mathrm{G}^{\mathrm{n}+1}$ and $\overline{\mathrm{G}}^{\mathrm{n}+1}$ are isomorphic as abstract group and the first $\ell$ basis vec- 
tors of $\Lambda$ corresponding to $U^{n+1}$ are mapped on the first $\ell$ basis vectors of $\bar{\Lambda}$ corresponding to $\overline{\mathrm{U}}^{\mathrm{n}+1}$. So $\mathrm{G}^{\mathrm{n}+1}$ and $\overline{\mathrm{G}}^{\mathrm{n}+1}$ are isomorphic as Galilean group. Proposition 17:

The Galilean space-time groups $\mathrm{G}^{\mathrm{n}+1}$ and $\overline{\mathrm{G}}^{\mathrm{n}+1}$ are isomorphic if and only if there are automorphisms $x \in \mathbb{N}_{\phi(K)}$ and $\omega \in$ Aut $K$ (5.4) such that $x$ is of the form (5.2) and relation (3.8) is satisfied:

$$
f_{i}\left(\bar{\varepsilon}_{1}, \ldots, \bar{g}_{r}\right)+\sum_{j=1}^{v} \pi_{i}\left(w \alpha_{j}\right) \cdot c\left(\alpha_{j}\right)=x B_{i} \quad(i=1, \ldots, r)
$$

Proof: If $\mathrm{G}^{\mathrm{n}+1}$ and $\overline{\mathrm{G}}^{\mathrm{n}+1}$ are isomorphic, there are automorphisms $X$ and $\omega$, because of proposition 7. Moreover the elements of $\underline{R}^{n} \cap \Lambda$ are mapped on elements of $\underline{R}^{n} \cap \bar{\Lambda}$. This means that $x$ is of the form $(5.2)$.

On the other hand, if $x$ and $\omega$ exist with the required properties, then $\mathrm{G}^{\mathrm{n}+1}$ and $\bar{G}^{\mathrm{n}+1}$ are isomorphic as abstract groups and every element of $\mathrm{U}^{\mathrm{n}+1}$ is mapped by $\psi$ on an element of the same type of $\bar{U}^{n+1}$ and vice versa. So both Galilean space-time groups are isomorphic.

\section{Conclusion}

In this part crystallographic groups in Euclidean, Minkowskian, Galilean and so-called product space haven been defined and equivalence relations between them stated. In Euclidean space two space groups are isomorphic if they are isomorphic as groups, but because of the existence of various kinds of elements in the other vector spaces, the isomorphism then is more complicated.

In each of the $n+1$-dimensional spaces mentioned a space-(time) group may be obtained from an extension of a free abelian group of rank $n+1$ by a point group $K$ with a monomorphism $\phi: K \rightarrow G L(n+1, Z)$. In Euclidean space every extension of this type with $K$ finite gives rise to a Euclidean space group $[3, p .557]$. A comparable proposition is not known for the other spaces, as there infinite point groups may occur. (If $\mathrm{K}$ is a finite crystallographic point group, imbedding theorems corresponding to proposition 5 of ref. [3] may be formulated in quite the same way).

For these reasons only those crystallographic groups are considered here for which the injection in the inhomogeneous linear group coincides with the injection of a generalized magnetic group. For Euclidean space this means that 
space groups are considered for which the point groups are $n+1$-reducible over R. Relativistic and Galilean space-time groups are considered only as far as they have finite point groups, i.e. in which no Lorentz or Galilean transformations of finite order occur.

Already for these simpler groups the classification is rather rich. The number of Euclidean and Galilean Bravais classes is finite, but the number of relativistic Bravais classes is infinite with the power of the continuum. In product space the number is enumerably infinite.

The space-time groups, considered here, being isomorphic as groups to a Euclidean space group, the abstract isomorphism classes of these space-time groups may be determined from all non-isomorphic extensions (1.1) where $K$ corresponds (according to fig. 1) to a generalized magnetic point group. 


\section{PART TWO: CENTRAL EXTENSIONS}

1. Introduction

A $n$-dimensional Euclidean space group $G$ may be obtained from an extension<smiles>[H][Y]#[Z20][Y]O</smiles>

of a free abelian group of rank $n$ by a finite group $K$ with a monomorphism $\phi: K \rightarrow G L(n, Z)[1][2]$.

To get the non-isomorphic space groups in $n$ dimensions one has to determine all non-equivalent extensions for one representative of each arithmetic crystal class and to identify the isomorphic extensions. In this part a procedure is given to determine the equivalence and isomorphism classes of extensions for a given arithmetic point group $\phi(K)$. Inequivalent extensions of an arbitrary abelian group $A$ by $K$, with $\phi: K \rightarrow A u t(A)$, are in one-to-one correspondence with the elements of the cohomology group $\mathrm{H}_{\phi}^{2}(\mathrm{~K}, A)$. If one uses a method indicated by $M$. Hall $[3, \mathrm{p} .230]$ for evaluating $\mathrm{H}_{\phi}^{2}(\mathrm{~K}, \mathrm{~A})$, one has to know the so called extension conditions. These are discussed in section 2 and are explicitly given in the appendix for the isomorphism classes of the so called four-dimensional generalized magnetic point groups, the four-dimensional crystallographic point groups which are $(3+1)$-reducible over $R$ [4].

The same problem becomes much simpler if one considers inequivalent extensions of $\mathrm{z}^{\mathrm{n}}$ instead of an arbitrary abelian group. In section 3 a method for the determination of $\mathrm{H}_{\phi}^{2}\left(\mathrm{~K}, \mathrm{z}^{\mathrm{n}}\right)$ is given. The final formulas turn out to bear a close resemblance to those of a paper by Zassenhaus [5] who worked out an algorithm for the determination of space groups by their non-primitive translations. The reason for this analogy can be found in an isomorphism between $\mathrm{H}_{\phi}^{2}\left(\mathrm{~K}, \mathrm{z}^{\mathrm{n}}\right)$ and $\mathrm{H}_{\phi}^{1}\left(\mathrm{~K}, \mathrm{R}^{\mathrm{n}} / \mathrm{Z}^{\mathrm{n}}\right)$ [2]. A short discussion of this aspect is given in section 4.

Because a space group may be given by generators and defining relations, by a factor system or by a system of non-primitive translations, it is worthwhile to consider the relations between these representations. This is done 
in section 5 .

Once the non-equivalent extensions are known the isomorphism classes have to be determined. To do this dealing with generators and defining relations, as found according to the method of section 3 , is in general not easy. A more systematic approach is possible by determining first inequivalent systems of non-primitive translations.

For a specific three-dimensional arithmetic point group the various techniques are illustrated by an example.

Using our method all non-equivalent four-dimensional generalized magnetic space-time groups have been determined. The results are available in the form of a technical report [6].

We consider here a group $\mathrm{K}$ which is finitely generated and presented. We use a multiplicative notation for $K$ and an additive one for $G$ and $z^{n}$. Of course this does not imply that $G$ is abelian. $K$ is generated by $\alpha_{1}, \ldots, \alpha_{\nu}$ with defining relations $\phi_{i}\left(\alpha_{1}, \ldots, \alpha_{v}\right)=\varepsilon \quad(i=1, \ldots, r)$. Here $\varepsilon$ is the unit element of $\mathrm{K}$.

$$
K=\left\langle\alpha_{1}, \ldots, \alpha_{v} ; \phi_{i}\left(\alpha_{1}, \ldots, \alpha_{v}\right), i=1, \ldots, r\right\rangle
$$

We suppose $K$ finite and we denote the order of the group by $k$.

2. The determination of $\mathrm{H}_{\phi}^{2}(\mathrm{~K}, \mathrm{~A})$

For an abelian group $A$ an extension

$$
0 \longrightarrow \mathrm{A} \stackrel{\mathrm{K}}{\longrightarrow} \mathrm{G} \stackrel{\sigma}{\longrightarrow} \mathrm{K} \longrightarrow 1 \quad(\phi)
$$

is defined by one of its factor systems m $\epsilon \mathrm{z}_{\phi}^{2}(\mathrm{~K}, \mathrm{~A})$ (group of 2-cocycles). We suppose of course that $(\phi, m)$ forms a system of mappings obeying A9 and A14 of ref. [1].

Equivalent extensions have cohomologous factor systems and conversely.

If $r(\alpha)$ is a representative of the coset of $k A$ in $G$ which is mapped on $\alpha \in K$ by $\sigma$ one has

$$
\phi_{i}\left(r\left(\alpha_{1}\right), \ldots, r\left(\alpha_{v}\right)\right)=\kappa g_{i} \in \kappa A
$$


If other representatives are chosen

$$
r^{\prime}(\alpha)=k c(\alpha)+r(\alpha)
$$

with $c \in C_{\phi}^{l}(K, A)$ (group of 1-cochains), there are elements $\pi_{i}\left(\alpha_{j}\right)$ of the group ring $\mathrm{ZK}$ fulfilling

$$
\phi_{i}\left(r^{\prime}\left(\alpha_{1}\right), \ldots, r^{\prime}\left(\alpha_{\nu}\right)\right)=\kappa \sum_{j=1}^{\nu} \pi_{i}\left(\alpha_{j}\right) c\left(\alpha_{j}\right)+\phi_{i}\left(r\left(\alpha_{1}\right), \ldots, r\left(\alpha_{v}\right)\right)
$$

According to Hall [7], [8] elements $g_{i}$ define an extension if and only if for all $\left\{h_{i}\right\}_{i=1}, \ldots, r$, with $h_{i} \in Z K$, for which

$$
\sum_{i=1}^{r} h_{i} \pi_{i}\left(\alpha_{j}\right)=0 \quad(\text { any } j=1, \ldots, v)
$$

also

$$
\sum_{i=1}^{r} h_{i} g_{i}=0
$$

The solutions $H=\left[h_{1}, \ldots, h_{r}\right]$ of (2.4) form a left $Z K$-module and are called extension conditions for the group $K$. In general this module is not free. To know the extension conditions one has to construct a set of generators of the module, which can be obtained as follows.

Because $h_{i} \in Z K$ and $\pi_{i}\left(\alpha_{j}\right) \in Z K$ one has

$$
h_{i}=\sum_{\alpha \in K} m_{i}(\alpha) \alpha, \quad m_{i}(\alpha) \in Z
$$

and

$$
\pi_{i}\left(\alpha_{j}\right)=\sum_{\beta \in K} n_{i}\left(\alpha_{j} ; \beta\right) \beta, \quad n_{i}\left(\alpha_{j} ; \beta\right) \in Z
$$

Notice that the coefficients $m_{i}(\alpha)$ have to be determined, whereas the $n_{i}\left(\alpha_{j} ; \beta\right)$ are already known by means of (2.3).

Substitution of (2.6) and (2.7) in (2.4) gives 


$$
\begin{aligned}
0 & =\sum_{i=1}^{r} \sum_{\alpha \in K} m_{i}(\alpha) \alpha \pi_{i}\left(\alpha_{j}\right) \\
& =\sum_{i=1}^{r} \sum_{\alpha, \beta \in K} m_{i}(\alpha) n_{i}(\alpha ; \beta) \alpha \beta
\end{aligned}
$$

If we define

$$
c_{j}(\alpha)=\sum_{i=1}^{r} \sum_{\beta \in K} m_{i}(\beta) n_{i}\left(\alpha_{j} ; \beta^{-1} \alpha\right) \in Z
$$

relation (2.4) may be written as

$$
\sum_{\alpha \in K} C_{j}(\alpha) \alpha=0
$$

Hence

$$
\mathrm{C}_{j}(\alpha)=0 \quad(\forall a \in K, \text { any } j=1, \ldots, v)
$$

(2.8) and (2.9) correspond to a set of $k v$ linear homogeneous equations with integral coefficients for the $\mathrm{kr}$ unknown integers $\mathrm{m}_{i}(\alpha)$. A set of generators for the solutions of (2.9) gives a set of generators for the ZK-module of solutions of (2.4).

For the isomorphism classes of four-dimensional generalized magnetic point groups (and therefore also for 1-, 2- and 3-dimensional crystallographic point groups) the coefficients $\pi_{i}\left(\alpha_{j}\right)$ appearing in (2.4) are zero-divisors. This property facilitates the work of finding the solutions. One can proceed in a way demonstrated here for the group $C_{n} \times C_{2}$.

This is the group (1.2):

$$
\mathrm{K}=\mathrm{C}_{\mathrm{n}} \times \mathrm{C}_{2} \stackrel{\simeq}{=}\left\langle\alpha, \beta ; \alpha^{\mathrm{n}}, \beta^{2}, \alpha \beta \alpha^{-1} \beta\right\rangle \text {. }
$$

The corresponding words in the group $\mathrm{G}$ are

$$
\begin{aligned}
& \Phi_{1}(r(\alpha))=\operatorname{nr}(\alpha) \\
& \Phi_{2}(r(\beta))=2 r(\beta) \\
& \Phi_{3}(r(\alpha), r(\beta))=r(\alpha)+r(\beta)-r(\alpha)+r(\beta) .
\end{aligned}
$$


36

One easily obtains:

$$
\begin{aligned}
& \Phi_{1}(\kappa c(\alpha)+r(\alpha))=k N_{\alpha} c(\alpha)+n r(\alpha) \text { with } N_{\alpha}=1+\alpha+\ldots+\alpha^{n-1} \\
& \Phi_{2}(\kappa c(\beta)+r(\beta))=k N_{\beta} c(\beta)+2 r(\beta) \text { with } N_{\beta}=1+\beta \\
& \Phi_{3}(k c(\alpha)+r(\alpha), k c(\beta)+r(\beta))=\kappa D_{\beta} c(\alpha)+\kappa Z_{\alpha \beta} c(\beta)+\Phi_{3}(r(\alpha), r(\beta)) \\
& \text { with } D_{\beta}=1-\beta \text { and } Z_{\alpha \beta}=\alpha+\beta
\end{aligned}
$$

This gives for the elements $\pi_{i}\left(\alpha_{j}\right)$ :

$$
\begin{array}{ll}
\pi_{1}(\alpha)=N_{\alpha} & \pi_{1}(\beta)=0 \\
\pi_{2}(\alpha)=0 & \pi_{2}(\beta)=N_{\beta} \\
\pi_{3}(\alpha)=D_{\beta} & \pi_{3}(\beta)=Z_{\alpha \beta}
\end{array}
$$

The equations (2.4) become

$$
\left\{\begin{array}{l}
h_{1} N_{\alpha}+h_{3} D_{\beta}=0 \\
h_{2} N_{\beta}+h_{3} z_{\alpha \beta}=0
\end{array}\right.
$$

The elements $N_{\alpha}, D_{\beta}, N_{\beta}$ and $Z_{\alpha \beta}$ are zero-divisors of $\mathrm{ZK}$. Multiplication on the right by $D_{\alpha}$ and $D_{\beta}$ respectively gives

$$
\left\{\begin{array}{lll}
h_{3} & D_{B} & D_{\alpha}=0 \\
h_{3} & z_{\alpha \beta} & D_{\beta}=0 .
\end{array} .\right.
$$

The left annulator of $D_{\beta} D_{\alpha}$ and of $z_{\alpha \beta} D_{\beta}$ is the ideal $\left\{\mu N_{\alpha}+\nu N_{\beta} \mid \mu, \nu \in Z K\right\}$. Substitution of this in (2.10) gives:

$$
\left\{\begin{array} { l } 
{ h _ { 1 } N _ { \alpha } + ( \mu N _ { \alpha } + v N _ { \beta } ) D _ { \beta } = 0 } \\
{ h _ { 2 } N _ { \beta } + ( \mu N _ { \alpha } + \nu N _ { \beta } ) z _ { \alpha \beta } = 0 }
\end{array} \text { or } \quad \left\{\begin{array}{l}
\left(h_{1}+\mu D_{\beta}\right) N_{\alpha}=0 \\
\left(h_{2}+\nu Z_{\alpha \beta}\right) N_{\beta}=-\mu N_{\alpha} Z_{\alpha \beta}=-\mu N_{\alpha} N_{\beta}
\end{array}\right.\right.
$$


Hence for the general element $H=\left[h_{1}, h_{2}, h_{3}\right]$ one has

$$
\begin{aligned}
& \mathrm{H}=\mathrm{KH}_{1}+\lambda \mathrm{H}_{2}+\mu \mathrm{H}_{3}+\mathrm{\nu H}_{4} \quad \text { with } \mathrm{K}, \lambda, \mu, \nu \in \mathrm{ZK} \quad \text { and } \\
& \mathrm{H}_{1}=\left[\mathrm{D}_{\alpha}, 0,0\right] \\
& \mathrm{H}_{2}=\left[0, \mathrm{D}_{B}, 0\right] \\
& \mathrm{H}_{3}=\left[-\mathrm{D}_{B},-\mathrm{N}_{\alpha}, \mathrm{N}_{\alpha}\right] \\
& \mathrm{H}_{4}=\left[0,-\mathrm{Z}_{\alpha \beta}, \mathrm{N}_{\beta}\right]
\end{aligned}
$$

$\left\{\mathrm{H}_{1}, \mathrm{H}_{2}, \mathrm{H}_{3}, \mathrm{H}_{4}\right\}$ is a minimal set of generators of the $\mathrm{ZK}$-module. However it is not a basis, because its elements are not linearly independent. In fact for arbitrary $\sigma, \tau \in \mathrm{ZK}$ :

$$
\tau D_{\beta} \mathrm{H}_{1}+\sigma \mathrm{N}_{B} \mathrm{H}_{2}+\tau \mathrm{D}_{\alpha} \mathrm{H}_{3}=0 \text {. }
$$

None of these coefficients has an inverse in $Z K$, whence the module is not free.

The method illustrated above has been used to calculate the extension conditions for all abstract generalized magnetic point groups in four dimensions. The results are given in the appendix.

If we put $\mathrm{H}_{j}=\left[\mathrm{h}_{1}^{\mathrm{j}}, \mathrm{h}_{2}^{\mathrm{j}}, \mathrm{h}_{3}^{\mathrm{j}}\right]$ the possible extensions of $\mathrm{A}$ by $\mathrm{K} \cong \mathrm{C}_{\mathrm{n}} \times \mathrm{C}_{2}$ are given by the solutions of

$$
\sum_{i=1}^{r} h_{i}^{j} B_{i}=0 \quad j=1, \ldots, 4, \quad g_{i} \in A
$$

Extensions associated to $\left\{g_{i}\right\}_{i=1, \ldots, r}$ and $\left\{\overline{\mathrm{g}}_{i}\right\}_{i=1, \ldots, r}$ are equivalent if and only if one has

$$
\bar{B}_{i}=\sum_{j=1}^{v} \pi_{i}\left(\alpha_{j}\right) c\left(\alpha_{j}\right)+g_{i} \quad \text { for } \quad c\left(\alpha_{j}\right) \in A \text {. }
$$

We recall that equivalent extensions give rise to isomorphic groups ( $\mathrm{G} \cong \bar{G})$. 
Further on we also indicate how to solve equations (2.12) and (2.13) but only in the special case of $\mathrm{A} \cong \mathrm{z}^{\mathrm{n}}$.

\section{Extensions of $\mathrm{z}^{\mathrm{n}}$}

In the special case that $A$ in (2.1) is a free abelian group of rank $n$, the theorem of $\mathrm{Hall}$ enables us to calculate the non-equivalent extensions without an explicit knowledge of the extension conditions.

If $K=\left\langle\alpha_{1}, \ldots, \alpha_{v} ; \phi_{i}\left(\alpha_{1}, \ldots, \alpha_{\nu}\right), i=1, \ldots, r\right\rangle$, the group $G$ is generated by

$$
\begin{aligned}
& a_{1}, \ldots, a_{n}, \quad \text { free generators of } z^{n} \\
& r\left(\alpha_{1}\right), \ldots, r\left(\alpha_{v}\right), \text { representatives of the cosets corresponding to } \alpha_{1}, \ldots, \alpha_{v}
\end{aligned}
$$
and defined by the relations

$$
\begin{array}{ll}
a_{i}+a_{j}=a_{j}+a_{i} & (i, j=1, \ldots, n) \\
r(\alpha)+a_{i}-r(\alpha)=\alpha a_{i} & (i=1, \ldots, n \quad \alpha \in K) \\
\phi_{i}\left(r\left(a_{1}\right), \ldots, r\left(\alpha_{v}\right)\right)=g_{i} & (i=1, \ldots, r)
\end{array}
$$

(We have here identified $\mathrm{z}^{\mathrm{n}}$ with its isomorphic image $\kappa \mathrm{z}^{\mathrm{n}}$ and written aa for $\phi(\alpha) a)$.

The elements $g_{i}$ are determined by equation (2.5). Because $\pi_{i}\left(\alpha_{j}\right)$ and $h_{i}$ are elements of the integral group ring $\mathrm{ZK}$, they are linear operators on $\mathrm{z}^{\mathrm{n}}$, and so they can be represented by $n \times n$-matrices with integral entries. From all matrices $h_{i}$ we form a matrix $h=\left(h_{1} h_{2} \ldots h_{r}\right)$.

Putting $\mathrm{p}=\mathrm{n} r, \mathrm{~h}$ is a $\mathrm{n} \times \mathrm{p}$ matrix.

From all matrices $\pi_{i}\left(\alpha_{j}\right)$ we form

$$
\pi=\left(\begin{array}{lll}
\pi_{1}\left(\alpha_{1}\right) & \ldots & \pi_{1}\left(\alpha_{v}\right) \\
\vdots & & \vdots \\
\pi_{r}\left(\alpha_{1}\right) & \ldots & \vdots \\
\pi_{r}\left(\alpha_{v}\right)
\end{array}\right)
$$


Putting $q=n v, \pi$ is a $p \times q$ matrix.

Furthermore we form the column vectors

$$
\begin{aligned}
& \Phi=\left(\begin{array}{c}
\mathrm{g}_{1} \\
\vdots \\
\vdots \\
\mathrm{g}_{\mathrm{r}}
\end{array}\right) \in \mathrm{z}^{\mathrm{p}} \text { and } \\
& \mathrm{C}=\left(\begin{array}{c}
\mathrm{c}\left(\alpha_{1}\right) \\
\vdots \\
\vdots \\
c\left(\alpha_{v}\right)
\end{array}\right) \quad \in \mathrm{z}^{\mathrm{q}}
\end{aligned}
$$

We call $\Phi$ a relation vector.

Using these definitions the equations (2.4) and (2.5) may be written respectively as:

$\mathrm{h} \Pi=0$

$\mathrm{h} \Phi=0$

An extension equivalent to one given by $\phi_{i}\left(r\left(\alpha_{1}\right), \ldots, r\left(\alpha_{v}\right)\right)=g_{i}$ is determined by

$$
\begin{aligned}
\phi_{i}\left(r^{\prime}\left(\alpha_{1}\right), \ldots, r^{\prime}\left(\alpha_{v}\right)\right) & =\phi_{i}\left(c\left(\alpha_{1}\right)+r\left(\alpha_{1}\right), \ldots, c\left(\alpha_{v}\right)+r\left(\alpha_{v}\right)\right)= \\
& =\sum_{j=1}^{v} \pi_{i}\left(\alpha_{j}\right) c\left(\alpha_{j}\right)+g_{i} \quad\left(c\left(\alpha_{j}\right) \in z^{n}, i=1, \ldots, r\right)
\end{aligned}
$$

So vectors $\Phi$ and $\Phi^{\prime}$ determine equivalent extensions if there is a $C \in \mathrm{Z}^{\mathrm{q}}$ such that:

$$
\Phi^{\prime}=\Pi \mathrm{C}+\Phi
$$

So the non-equivalent extensions are determined by the solutions of (3.6) modulo $\pi z^{\mathrm{q}}$.

Consider now the natural imbedding $i: z \rightarrow R$. Define 


$$
D=\pi R^{q} \subseteq R^{p}
$$

$D$ is a s-dimensional real vector space generated by the column vectors:

$$
\left(\begin{array}{l}
\pi_{11} \\
\vdots \\
\pi_{\mathrm{p} 1}
\end{array}\right) \quad \cdots \cdots\left(\begin{array}{l}
\pi_{1 q} \\
\vdots \\
\pi_{\mathrm{pq}}
\end{array}\right)
$$

so that $\mathbf{s}$ is also the rank of $\pi$.

If we consider the row vectors $\left(h_{11} \ldots h_{1 p}\right), \ldots,\left(h_{n}, \ldots, h_{n p}\right)$, condition (3.5) implies: these $n$ vectors are in the orthogonal complement (in the usual Euclidean metric) of $D$ in $R^{p}$. So $D$ is orthogonal to each $\left(h_{i 1}, \ldots, h_{i p}\right)$. Hence each $\Phi \in D$ fulfils condition (3.6).

On the other hand each solution of (3.6) is in the orthogonal complement of the space generated by $\left(h_{11}, \ldots, h_{1 p}\right), \ldots,\left(h_{n 1}, \ldots, h_{n p}\right)$, i.e. in the closure $\bar{D}=D$.

So each point in $\mathrm{D} \cap \mathrm{z}^{\mathrm{p}}$ determines an extension. Conversely each extension is represented by a point of $D \cap z^{p}$.

If $\Phi_{1}$ and $\Phi_{2} \in \mathrm{D} \cap \mathrm{Z}^{\mathrm{P}}$, also $\Phi_{1}+\Phi_{2} \in \mathrm{D} \cap \mathrm{Z}^{\mathrm{P}}$. As explained in section 5 an element $\Phi$ determines a factor system m only up to $\delta c$ with $c \in C_{\phi}^{l}\left(K, z^{n}\right)$ such that $c\left(\alpha_{1}\right)=\ldots=c\left(\alpha_{v}\right)=0$.

Define

$$
\widetilde{\mathrm{B}}_{\phi}^{2}\left(K, \mathrm{Z}^{\mathrm{n}}\right)=\left\{\delta c \mid c \in \mathrm{C}_{\phi}^{1}\left(K, \mathrm{Z}^{\mathrm{n}}\right) \text { with } c\left(\alpha_{1}\right)=c\left(\alpha_{\nu}\right)=0\right\}
$$

If $\Phi_{1}$ and $\Phi_{2}$ determine factor sets $m_{1}$ and $m_{2}$ respectively (modulo $\widetilde{B}_{\phi}^{2}\left(K, z^{n}\right)$ ), then $\Phi_{1}+\Phi_{2}$ determines an extension with factor set $\left(m_{1}+m_{2}\right)\left(\bmod \widetilde{B}_{\phi}^{2}\left(K, z^{n}\right)\right)$. Hence

$$
\mathrm{D} \cap \mathrm{z}^{\mathrm{P}} \stackrel{\cong}{=} \mathrm{z}_{\phi}^{2}\left(\mathrm{~K}, \mathrm{z}^{\mathrm{n}}\right) / \tilde{\mathrm{B}}_{\phi}^{2}\left(\mathrm{~K}, \mathrm{z}^{\mathrm{n}}\right)
$$

Because of (3.7) all extensions equivalent to the split extension are obtained from

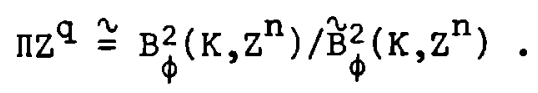


Therefore all non-equivalent extensions are given by

$D \cap z^{P}\left(\bmod \pi z^{q}\right) \cong z_{\phi}^{2}\left(K, z^{n}\right) / B_{\phi}^{2}\left(K, z^{n}\right)$

From this follows

Proposition 1:

The non-equivalent $(K, \phi)$-extensions of $\mathrm{z}^{\mathrm{n}}$ are in one-to-one-correspondence with the points of

$$
\left(\pi R^{q}\right) \cap z^{p}\left(\bmod \pi z^{q}\right) \stackrel{\simeq}{=} H_{\phi}^{2}\left(K, z^{n}\right)
$$

Corollary 1: For finite $K$ the second cohomology group $\mathrm{H}_{\phi}^{2}\left(\mathrm{~K}, \mathrm{z}^{\mathrm{n}}\right)$ is a finite abelian group.

Proof: The elements of $\pi \mathrm{z}^{\mathrm{q}}$ form a s-dimensional lattice in a Euclidean space of the same dimension. Therefore the volume of the unit cell is finite. In such a finite volume there is only a finite number of the discrete points $\mathrm{D} \cap \mathrm{z}^{\mathrm{p}}$.

The number of these points being the order of $\mathrm{H}_{\phi}^{2}\left(K, \mathrm{z}^{\mathrm{n}}\right)$, this group is finite. It is clear that it is abelian. So $\mathrm{H}_{\phi}^{2}\left(\mathrm{~K}, \mathrm{z}^{\mathrm{n}}\right)$ is characterized by a finite number of torsion numbers.

This is a well-known result and has been proved already in another way.

To determine the elements of the group (3.11) one applies automorphisms $P \in G L(p, Z)$ and $Q \in G L(q, Z)$ in such a way that

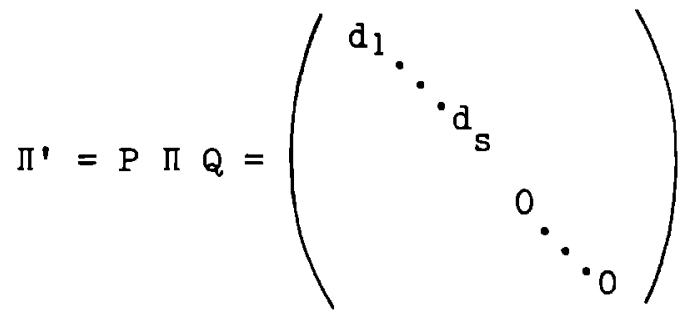

This can be achieved, because according to the theorem of elementary divisors $[9, p .101]$ it is even possible to bring $\Pi$ ' in the form (3.12) with positive $a_{1}\left|d_{2}\right| \ldots \mid d_{s}$. If $d_{i}>0(i=1, \ldots, s)$ the elements of

$$
\left(\Pi^{\prime} \mathrm{R}^{\mathrm{q}}\right) \cap \mathrm{z}^{\mathrm{p}} \quad\left(\bmod \Pi^{\prime} \mathrm{z}^{\mathrm{q}}\right)
$$


are given by vectors in $Z^{P}$. Suppose $v^{\prime}$ is such a vector then its possible components are

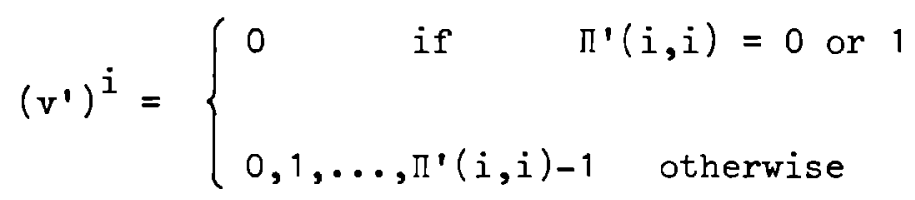

Then the corresponding element $v$ of $(3.11)$ is given by

$$
v=P^{-1} v^{\prime}
$$

So $\mathrm{H}_{\phi}^{2}\left(\mathrm{~K}, \mathrm{Z}^{\mathrm{n}}\right)$ is isomorphic to the abelian group generated by the vectors

$$
e_{i}=P^{-1} \varepsilon_{i} \quad\left(i=1, \ldots, s ; d_{i}>1\right)
$$

(where $\varepsilon_{i}$ is the element of $z^{p}$ with $i^{\text {th }}$ component 1 and all other components zero) and defined by relations

$$
d_{i} e_{i}=0 \quad\left(\text { any } i \text { for which } d_{i}>1\right)
$$

From this it follows that the group structure of $\mathrm{H}_{\phi}^{2}\left(\mathrm{~K}, \mathrm{z}^{\mathrm{n}}\right)$ is given by the torsion numbers $d_{i}>1$.

4. The connection between the cohomology groups $H_{\phi}^{2}\left(K, z^{n}\right)$ and $H_{\phi}^{l}\left(K, R^{n} / Z^{n}\right)$

To see the relation of our formula (3.11) with the Zassenhaus formulas [5] one considers the connection between a factor system and the corresponding system of non-primitive translations. This aspect is extensively discussed in ref. [2] . We limit ourselves here to some general remarks.

Consider the following morphism of group extensions

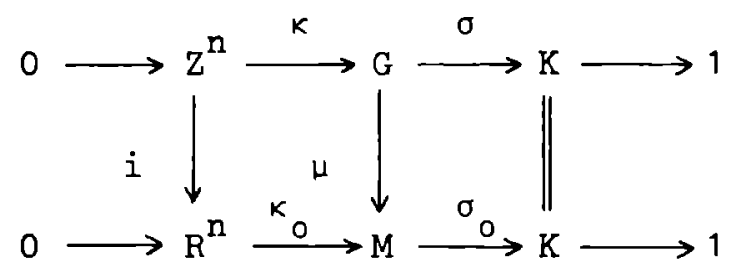


where $\phi: K \rightarrow G L(n, R)$ is the linear continuation of $\phi: K \rightarrow G L(n, Z)$ and $i$ is the natural imbedding $z^{n} \rightarrow R^{n}$. If $K$ is finite, the lower extension splits because $R^{n}$ is divisible and torsion free. We choose $M$ to have a trivial factor system $m_{0}$. We denote the representatives of the cosets of $\kappa_{0} R^{n}$ in $M$ by $r_{0}(\alpha)$. Then the monomorphism $\mu$ is given by

$$
\begin{array}{ll}
\mu \kappa a=\kappa_{0} i a, & \left(\forall a \in z^{n}\right) \\
\mu r(\alpha)=\kappa_{0} u(\alpha)+r_{0}(\alpha), & \left(u(\alpha) \in R^{n}\right)
\end{array}
$$

$u$ is a 1-cochain: $u \in C_{\phi}^{l}\left(K, R^{n}\right)$, which may be defined in the following way. The exact sequence (with $i$ as above)

$$
0 \longrightarrow \mathrm{z}^{\mathrm{n}} \stackrel{\mathrm{i}}{\longrightarrow} \mathrm{R}^{\mathrm{n}} \stackrel{\pi}{\longrightarrow} \mathrm{R}^{\mathrm{n}} / \mathrm{z}^{\mathrm{n}} \longrightarrow 0
$$

induces the following commutative diagram with exact rows and columns

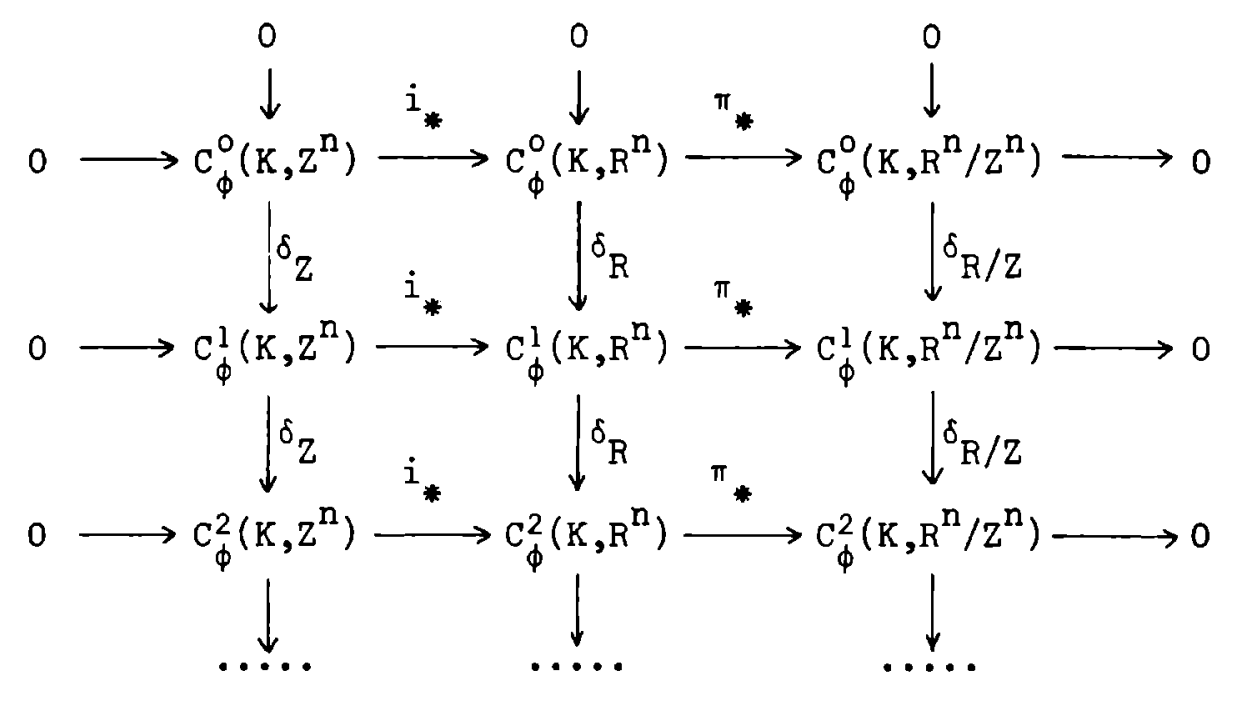

If $m \in z_{\phi}^{2}\left(K, z^{n}\right)$ is a factor system, $i_{*} m \in z_{\phi}^{2}\left(K, R^{n}\right)=B_{\phi}^{2}\left(K, R^{n}\right)$, because $\mathrm{H}_{\phi}^{2}\left(K, \mathrm{R}^{\mathrm{n}}\right)^{\phi}=0$. Then there exists an element $\mathrm{u} \in \mathrm{C}_{\phi}^{\mathrm{l}}\left(\mathrm{K}, \mathrm{R}^{\mathrm{n}}\right)$ with

$$
\delta_{\mathrm{R}} \mathrm{u}=\mathrm{i}_{*} \mathrm{~m}
$$

$u$ forms a system of non-primitive translations. One can show that $\delta_{R / Z}{ }_{*} u=0$. 
Then $u$ modulo $i z^{\mathrm{n}}$ is a crossed homomorphism:

$$
u(\alpha \beta) \equiv u(\alpha)+\alpha u(\beta) \quad\left(\bmod i z^{n}\right) \quad(\forall \alpha, \beta \in K)
$$

$u$ is only determined modulo $z_{\phi}^{l}\left(K, R^{n}\right)$. If $v \in z_{\phi}^{l}\left(K, R^{n}\right)=B_{\phi}^{l}\left(K, R^{n}\right)$ there is an element $f \in C_{\phi}^{0}\left(K, R^{n}\right)$ with $\delta_{R} f=v$ and $\delta_{R}(u+v)=\delta_{R} u=i_{*}$. For an equivalent factor system $m^{\prime}=m+\delta_{z} c$, with $c \in C_{\phi}^{l}\left(K, z^{n}\right)$, one has

$$
i_{*} m^{\prime}=\delta_{R}\left(u+i_{*} c\right)
$$

because $i_{*} \delta_{z} c=\delta_{R} i_{*} c$.

So if $\mathrm{m}$ and $\mathrm{m}$ ' are cohomologous one has for corresponding systems of nonprimitive translations: $i_{*} m=\delta_{R} u$ and $i_{*} m^{\prime}=\delta_{R} u^{\prime}$ the relation

$$
u^{\prime}=u+\delta_{R} f+i_{*} c
$$

This is the equivalence relation between systems of non-primitive translations, called "Starke Aequivalenz" by Zassenhaus.

Consider now $\pi_{*} u$ and $\pi_{*} u^{\prime}$ for $u$ and $u^{\prime}$ as in $(4.5) \cdot \pi_{*} u \in z_{\phi}^{l}\left(K, R^{n} / z^{n}\right)$ because $\delta_{\mathrm{R} / \mathrm{z}} \pi_{*} \mathrm{u}=\pi_{*} \delta_{\mathrm{R}} \mathrm{u}=\pi_{*} i_{*} \mathrm{II}=0$. Furthermore $\pi_{*}$ maps coboundaries on coboundaries. $\pi_{*} u$ and $\pi_{*} u$ ' belong to the same cohomology class because:

$$
\left[\pi_{*} u^{\prime}\right]=\left[\pi_{*} u+\pi_{*} \delta_{R} f\right]=\left[\pi_{*} u\right] \in H_{\phi}^{l}\left(K, R^{n} / z^{n}\right)
$$

In fact $\pi_{*} \delta_{R} f=\delta_{R} / Z^{\pi} f$ and belongs therefore to $B_{\phi}^{l}\left(K, R^{n} / Z^{n}\right)$. Conversely if $\left[\pi_{*} u\right]=\left[\pi_{*} u^{\prime}\right]$ one has the relation (4.5) between $u$ and $u^{\prime}$. So $[\mathrm{m}]=\left[\mathrm{m}^{\mathrm{l}}\right] \in \mathrm{H}_{\phi}^{2}\left(\mathrm{~K}, \mathrm{z}^{\mathrm{n}}\right)$. This one-to-one correspondence between the elements of $H_{\phi}^{l}\left(K, R^{n} / Z^{n}\right)$ and $H_{\phi}^{2}\left(K, z^{n}\right)$ is an isomorphism. As one sees from the long exact sequence

$$
\cdots \mathrm{H}_{\phi}^{1}\left(\mathrm{~K}, \mathrm{z}^{\mathrm{n}}\right) \stackrel{\mathrm{i}_{*}}{\longrightarrow} \mathrm{H}_{\phi}^{1}\left(\mathrm{~K}_{0}, \mathrm{R}^{\mathrm{n}}\right) \stackrel{\pi_{*}}{\longrightarrow} \mathrm{H}_{\phi}^{1}\left(\mathrm{~K}, \mathrm{R}^{\mathrm{n}} / \mathrm{z}^{\mathrm{n}}\right) \stackrel{\delta_{*}}{\longrightarrow} \mathrm{H}_{\phi}^{2}\left(\mathrm{~K}, \mathrm{z}^{\mathrm{n}}\right) \stackrel{\mathrm{i}_{*}}{\longrightarrow} \mathrm{H}_{\phi}^{2}\left(\mathrm{~K}, \mathrm{R}^{\mathrm{n}}\right) \rightarrow
$$

$\delta_{*}$ is in our case an isomorphism (the connecting isomorphism). One verifies that $u$ and $m$ as above are related according to: 


$$
\delta_{*}\left[\pi_{*} \mathrm{u}\right]=[\mathrm{m}]
$$

If $K=\left\langle\alpha_{1}, \ldots, \alpha_{v} ; \phi_{i}\left(\alpha_{1}, \ldots, \alpha_{v}\right\rangle, i=1, \ldots, r\right\rangle(1.2)$, a system $u$ of nonprimitive translations is defined already by $u\left(\alpha_{1}\right), \ldots, u\left(\alpha_{v}\right)$ using relation (4.4). In this case, as Zassenhaus has shown [5], u is uniquely determined, (modulo $\mathrm{z}^{\mathrm{n}}$ ), if and only if one has

$$
\sum_{j=1}^{v} \pi_{i}\left(\alpha_{j}\right) u\left(\alpha_{j}\right) \equiv 0 \quad\left(\bmod i z^{n}\right) \quad(i=1, \ldots, r)
$$

If we introduce the vector

$$
U=\left(\begin{array}{l}
u\left(\alpha_{1}\right) \\
\vdots \\
\vdots \\
u\left(\alpha_{v}\right)
\end{array}\right) \quad \in R^{q} \text {, }
$$

then (4.9) may be written in matrix formulation:

$$
\pi U \equiv 0 \quad\left(\bmod z^{p}\right)
$$

The solutions of this congruence may be determined in the same way as done for (3.11).

5. Relation between the vectors $U$ and $\Phi$, the factor system $m$ and the system of non-primitive translations $u$

We consider the groups $G$ appearing in (4.1) for fixed lower extension (thus also fixed $\phi(K)$ ).

Then $G$ is determined if one knows the factor system $\mathrm{m}$, or the relation vector $\Phi$, or the system of non-primitive translations $u$, or even the non-primitive translations vector $U$. We now discuss the mutual relations between these various ways of presenting a given group $G$.

Case 1: A factor system m is given.

This means that writing the elements of $G$ as: 


$$
(a, \alpha)=k a+r(\alpha) \quad\left(\forall a \in z^{n}\right) \quad(\forall \alpha \in K)
$$

the product of any two elements is given by:

$$
(a, \alpha)+(b, \beta)=(a+\alpha b+m(\alpha, \beta), \alpha \beta)
$$

\section{i) Find $\Phi$ :}

Using (5.1) one easily calculates the uniquely determined elements

$$
\kappa g_{i}=\Phi_{i}\left(r\left(\alpha_{1}\right), \ldots, r\left(\alpha_{v}\right)\right) \quad(i=1,2, \ldots, r)
$$

and the corresponding relation vector $\Phi$.

ii) Find $u$ :

We have already seen, that for given $m$ there exists a system $u$ of non-primitive translations, such that $i_{*} m=\delta_{R} u$. This means:

$$
i \mathbb{m}(\alpha, \beta)=u(\alpha)+\alpha u(\beta)-u(\alpha \beta), \quad \forall \alpha, \beta \in K
$$

From this it follows that, with $k=|K|$ and $s=\underset{\beta \in K}{\sum} u(\beta)$ :

$$
\text { i } \sum_{\beta \in \mathrm{K}} \mathrm{m}(\alpha, \beta)=\mathrm{ku}(\alpha)+(\alpha-1) \mathrm{s} \text {. }
$$

Because $\mathrm{R}^{\mathrm{n}}$ is divisible and torsion free

$$
u(\alpha)=\frac{1}{k} \text { i } \sum_{\beta \in K} m(\alpha, \beta)+(1-\alpha) f \quad \text { with } \quad f=\frac{s}{k} \in R^{n}
$$

Now:

$$
u^{\prime}(\alpha)=\frac{1}{k} i \underset{\beta \in K}{\Sigma} m(\alpha, \beta)
$$

also gives a system of non-primitive translations corresponding to $m$ (in fact $i_{*} m=\delta_{R} u^{\prime}$, because $\left.u^{\prime}=u+\delta_{R} f\right)$. Relation (5.5) represents therefore a solution of the problem. From (5.4) and (5.5) one sees that the factor system $m$ determines a system of non-primitive translations $u$ only modulo a principal crossed homomorphism (i.e. an element of $B_{\phi}^{1}\left(K, R^{n}\right)$ ). 
iii) Find $U$ :

The vector $U$ trivially follows from the knowledge of the system of non-primitive translations $u$.

\section{Case 2: The relation vector $\Phi$ is given.}

This means that one knows the elements $g_{i}$ through (3.3).

i) Find $m$ :

According to $[3, p .227]$ if the elements $g_{i}$ are known, then elements $\gamma_{i}(\alpha, \beta) \in Z K$ can be found such that

$$
m(\alpha, \beta)=\sum_{i=1}^{r} \gamma_{i}(\alpha, \beta) g_{i},
$$

where of course $k m(\alpha, \beta)=r(\alpha)+r(\beta)-r(\alpha \beta)$.

For the determination of the $\gamma_{i}(\alpha, \beta)$, the words $(r(\alpha)+r(\beta)-r(\alpha \beta))$ have to be expressed as a sum of conjugates of the words $\Phi_{i}\left(r\left(\alpha_{1}\right), \ldots, r\left(\alpha_{v}\right)\right)$. Present each $\alpha \in K\left(\alpha \neq \alpha_{1}, \ldots, \alpha_{v}\right)$ by a fixed word $w_{\alpha}\left(\alpha_{1}, \ldots, \alpha_{v}\right)$ and choose as representative $r(\alpha)$ the corresponding word in the $r\left(\alpha_{j}\right)(j=1, \ldots, v)$

$$
r(\alpha)=w_{\alpha}\left(r\left(\alpha_{1}\right), \ldots, r\left(\alpha_{v}\right)\right)
$$

For a generator $\alpha_{j}(j=1, \ldots, v)$ one has trivially

$$
w_{\alpha_{j}}\left(r\left(\alpha_{1}\right), \ldots, r\left(\alpha_{v}\right)\right)=r\left(\alpha_{j}\right)
$$

Then

$$
\kappa m(\alpha, \beta)=w_{\alpha}+w_{\beta}-w_{\alpha \beta} \stackrel{\text { Def }}{=} w_{\alpha, \beta}\left(r\left(\alpha_{1}\right), \ldots, r\left(\alpha_{\nu}\right)\right), \quad(\forall \alpha, \beta \in K)
$$

Because

$$
w_{\alpha, \beta}\left(\alpha_{1}, \ldots, \alpha_{v}\right)=\epsilon
$$

one has

$$
W_{\alpha, B}\left(\alpha_{1}, \ldots, \alpha_{v}\right)=\Pi \alpha_{j_{1}} \ldots \alpha_{j_{t}} \Phi_{j}\left(\alpha_{1}, \ldots, \alpha_{v}\right) \alpha_{j_{t}}^{-1} \ldots \alpha_{j_{1}}^{-1}
$$

Hence 


$$
m(\alpha, \beta)=\sum_{j} \alpha_{j_{1}} \cdots \alpha_{j_{t}} g_{j}=\sum_{i=1}^{r} \gamma_{i}(\alpha, \beta) g_{i},
$$

and this gives a solution of the problem.

Although $\gamma_{i}(\alpha, \beta)$ is not unique, $m(\alpha, \beta)$ is uniquely determined by the particular choice $(5.7)$.

Any other choice in the representatives $r(K)$ may be considered as follows. We suppose

$$
r^{\prime}\left(\alpha_{j}\right)=k \bar{c}\left(\alpha_{j}\right)+r\left(\alpha_{j}\right) \quad j=1, \ldots, v
$$

Then from $\phi_{i}\left(r\left(\alpha_{1}\right), \ldots, r\left(\alpha_{v}\right)\right)=\phi_{i}\left(r^{\prime}\left(\alpha_{1}\right) \ldots r^{\prime}\left(\alpha_{v}\right)\right)=g_{i} \quad(i=1, \ldots, r)$ and using (3.7) follows the relation:

$$
\Pi \bar{C}=0
$$

According to proposition 3 demonstrated below, (5.11) implies that there is a uniquely determined 1-cocycle $c \in \mathrm{Z}_{\phi}^{l}\left(K, \mathrm{z}^{\mathrm{n}}\right)$ with $c\left(\alpha_{j}\right)=\bar{c}\left(\alpha_{j}\right) \quad(j=1, \ldots, v)$. Defining

$$
\tilde{C}_{\phi}^{l}\left(K, z^{n}\right)=\left\{c \in C_{\phi}^{l}\left(K, z^{n}\right) \mid c\left(\alpha_{1}\right)=c\left(\alpha_{2}\right)=\ldots=c\left(\alpha_{\nu}\right)=0\right\}
$$

the 1 -cochain $\bar{c}$ in $(5.10)$ may be written as

$$
\bar{c}=\bar{c}_{1}+\bar{c}_{2}
$$

with $\bar{c}_{1} \in \mathrm{z}_{\phi}^{l}\left(\mathrm{~K}, \mathrm{z}^{\mathrm{n}}\right)$ and $\overline{\mathrm{c}}_{2} \in \tilde{\mathrm{C}}_{\phi}^{\mathrm{l}}\left(\mathrm{K}, \mathrm{z}^{\mathrm{n}}\right)$.

So the change in the factor system corresponding to the change in representatives $(5.10)$ is

$$
m^{\prime}=m+\delta_{z} \bar{c}=m+\delta_{z} \bar{c}_{2} \quad \text { with } \quad \delta_{z} \bar{c}_{2} \in \widetilde{B}_{\phi}^{2}\left(K, z^{n}\right)
$$

This shows that $\Phi$ determines m only modulo $\underset{\phi}{\tilde{\mathrm{B}}} \underset{\phi}{2}\left(\mathrm{~K}, \mathrm{z}^{\mathrm{n}}\right)$.

Proposition 2:

Given a word $w=w\left(\alpha_{1}, \ldots, \alpha_{v}\right)$ in the generators of $K$ and elements $\pi_{w}\left(\alpha_{j}\right)$ $(j=1, \ldots, v)$ of the group ring $\mathrm{ZK}$ defined by 


$$
v\left(w\left(\alpha_{1}, \ldots, \alpha_{v}\right)\right)=\sum_{j=1}^{v} \pi_{w}\left(\alpha_{j}\right) v\left(\alpha_{j}\right)
$$

for any crossed homomorphism $v \in \mathrm{z}_{\phi}^{l}\left(\mathrm{~K}, \mathrm{z}^{\mathrm{n}}\right)$. Then one has:

$$
\begin{aligned}
w\left(c\left(\alpha_{1}\right)+r\left(\alpha_{1}\right), \ldots, c\left(\alpha_{v}\right)\right. & \left.+r\left(\alpha_{\nu}\right)\right)=\sum_{j=1}^{\nu} \pi_{w}\left(\alpha_{j}\right) c\left(\alpha_{j}\right)+ \\
& +w\left(r\left(\alpha_{1}\right), \ldots, r\left(\alpha_{v}\right)\right)
\end{aligned}
$$

for any $c\left(\alpha_{j}\right) \in z^{n},(j=1, \ldots, \nu)$.

The converse is also true.

Proof: The proof is trivial for words of length one. If the proposition is true for $w$, one easily verifies it for words of the form $\alpha_{j}^{ \pm}{ }_{w}$. The result follows by induction.

Corollary 2: For $\pi_{\phi_{i}}\left(\alpha_{j}\right)$ as in proposition 2 and $\pi_{i}\left(\alpha_{j}\right)$ as defined by (2.3) one has:

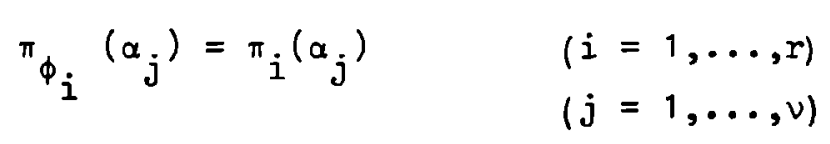

Proposition 3:

Consider the elements $\pi_{i}\left(\alpha_{j}\right)$ as above. Then the solutions $\left\{\bar{c}\left(\alpha_{1}\right), \ldots, \bar{c}\left(\alpha_{v}\right) \mid\right.$ $\left.\bar{c}\left(\alpha_{j}\right) \in z^{n}, j=1, \ldots, v\right\}$ of the system of equations

$$
\sum_{j=1}^{v} \pi_{i}\left(a_{j}\right) \bar{c}\left(\alpha_{j}\right)=0 \quad(i=1, \ldots, r)
$$

are in one-to-one correspondence with the 1-cocycles $c \in \mathrm{z}_{\phi}^{l}\left(\mathrm{~K}, \mathrm{z}^{\mathrm{n}}\right)$; in particular one has:

$$
c\left(\alpha_{j}\right)=\bar{c}\left(\alpha_{j}\right) \quad(j=1, \ldots, v)
$$

Proof: See [5, p.123-125]. Actually in this paper of Zassenhaus the proof is given for $\mathrm{R}^{\mathrm{n}} / \mathrm{Z}^{\mathrm{n}}$ instead of for $\mathrm{Z}^{\mathrm{n}}$, but this is not relevant. Let us remark that propositions 2 and 3 are also valid if one considers an abelian group A instead of the free abelian group $\mathrm{Z}^{\mathrm{n}}$. So the shown relations between $m$ and $\left\{g_{i}\right\}$ are also valid in the case of an arbitrary abelian group $A$ and only depend on the upper extension. 


\section{ii) Find $U$ :}

According to diagram (4.1) and considering $M$ presented as semidirect product of $R^{n}$ by $K$ (i.e. with trivial factor system $m_{0}$ ), one has:

$$
\begin{aligned}
i g_{j} & =i \Phi_{j}\left(r\left(\alpha_{1}\right), \ldots, r\left(\alpha_{v}\right)\right)=\Phi_{j}\left(u\left(\alpha_{1}\right)+r_{0}\left(\alpha_{1}\right), \ldots, u\left(\alpha_{v}\right)+r_{o}\left(\alpha_{v}\right)\right)= \\
& =\sum_{k=1}^{\nu} \pi_{j}\left(\alpha_{k}\right) u\left(\alpha_{k}\right) \quad(j=1, \ldots, r)
\end{aligned}
$$

This because $\Phi_{j}\left(r_{0}\left(\alpha_{1}\right), \ldots, r_{0}\left(\alpha_{v}\right)\right)=0$. Hence in terms of $(3.2),(3.3)$ and $(4.10)$, relation $(5.18)$ can be written as:

$$
\Phi=\Pi U
$$

Consider now $\Pi^{\prime}=P \Pi Q$ as in (3.12) with $P \in G L(p, Z)$ and $Q \in G L(q, z)$ and define:

$$
U^{\prime}=Q^{-1} U, \quad \Phi^{\prime}=P \Phi
$$

Then one obtains from (5.19)

$$
\Pi^{\prime} U^{\prime}=\Phi^{\prime}
$$

This implies that $\Phi^{\prime}$ is of the form

$$
\Phi^{\prime}=\left(\begin{array}{c}
g_{1}^{\prime} \\
\vdots \\
g_{S}^{\prime} \\
0 \\
\vdots \\
0
\end{array}\right) \quad \text { for some given } g_{1}^{\prime}, \ldots, g_{s}^{\prime} \in z
$$

Then 


$$
U^{\prime}=\left(\begin{array}{c}
g_{1}^{\prime} / d_{l} \\
\vdots \\
E_{s}^{\prime} / d_{s} \\
x_{s+1} \\
\vdots \\
x_{q}
\end{array}\right) \quad \text { with arbitrary } x_{s+1}, \ldots, x_{q} \in R
$$

and one gets $U$ from (5.20).

The arbitrariness of $U$ for given $\Phi$ simply expresses the arbitrariness in the imbedding $\mu$ of $(4.1)$ and corresponds to different choices of the origin [2].

In fact the $\mathrm{R}^{\mathrm{q}}$ vector

$$
V^{\prime}=\left(\begin{array}{l}
0 \\
\vdots \\
0 \\
x_{s+1} \\
\vdots \\
x_{q}
\end{array}\right) \quad \text { defines by means of } V=Q V^{\prime}
$$

a solution of the system of equations

$$
\sum_{j=1}^{v} \pi_{i}\left(\alpha_{j}\right) v\left(\alpha_{j}\right)=0 \quad(i=1, \ldots, r)
$$

According to proposition 3 ( for $A=R^{n}$ ) we may identify the $v\left(\alpha_{j}\right.$ ) with the corresponding values of a 1 -cocycle $v \in Z_{\phi}^{l}\left(K, R^{n}\right)=B_{\phi}^{l}\left(K, R^{n}\right)$.

Hence one can always define an equivalent system of non-primitive translations such that

$$
U^{\prime} \sim U^{\prime \prime}=\left(\begin{array}{c}
\mathrm{g}_{1}^{\prime} / \mathrm{d}_{1} \\
\vdots \\
\mathrm{g}_{\mathrm{s}}^{\prime} / \mathrm{d}_{\mathrm{s}} \\
0 \\
\vdots \\
0
\end{array}\right)
$$


for some $g_{i}^{\prime}, \ldots, g_{S}^{\prime} \in Z$ and $0 \leqslant g_{i}^{\prime}<d_{i}$.

iii) Find u:

We may suppose that we already know $u\left(\alpha_{1}\right) \ldots u\left(\alpha_{\nu}\right)$ (i.e. a vector $U$ ). By means of relation (4.4) we are then able to extend this set to a system $u$ of non-primitive translations.

Choose for any $\alpha \in K\left(\alpha \neq \alpha_{1}, \ldots, \alpha_{v}\right)$ a fixed word $w_{\alpha}\left(\alpha_{1}, \ldots, \alpha_{v}\right)$ and express

$$
u(\alpha)=u\left(w_{\alpha}\left(\alpha_{1}, \ldots, \alpha_{v}\right)\right)
$$

in $u\left(\alpha_{1}\right), \ldots, u\left(\alpha_{\nu}\right)$. It follows that $u$ is unique up to an element $c \in \tilde{C}_{\phi}^{l}\left(K, z^{n}\right)$. Explicitly one has (using (5.13)):

$$
u(\alpha)=\sum_{j=1}^{v} \pi_{w_{\alpha}}\left(\alpha_{j}\right) u\left(\alpha_{j}\right)+c(\alpha), \quad\left(\alpha \neq \alpha_{1}, \ldots, \alpha_{v}\right)
$$

Case 3: A system of non-primitive translations $u$ is given.

In this case $U$ is of course also given. The factor system can be uniquely found according to (4.3) by means of:

$$
i m(\alpha, \beta)=u(\alpha)+\alpha u(\beta)-u(\alpha \beta)
$$

One then obtains $\Phi$ following case 1 (i).

Case 4: A non-primitive vector $U$ is given.

We have already discussed (case 2 (iii)) how u can be obtained from U; using (5.26) one gets the factor system $\mathrm{m}$. The vector $\Phi$ is uniquely determined by (5.19).

\section{Conclusion}

The four different ways to define a group $G$ appearing in a diagram (4.1) have relations which can be summarized by the following diagram:

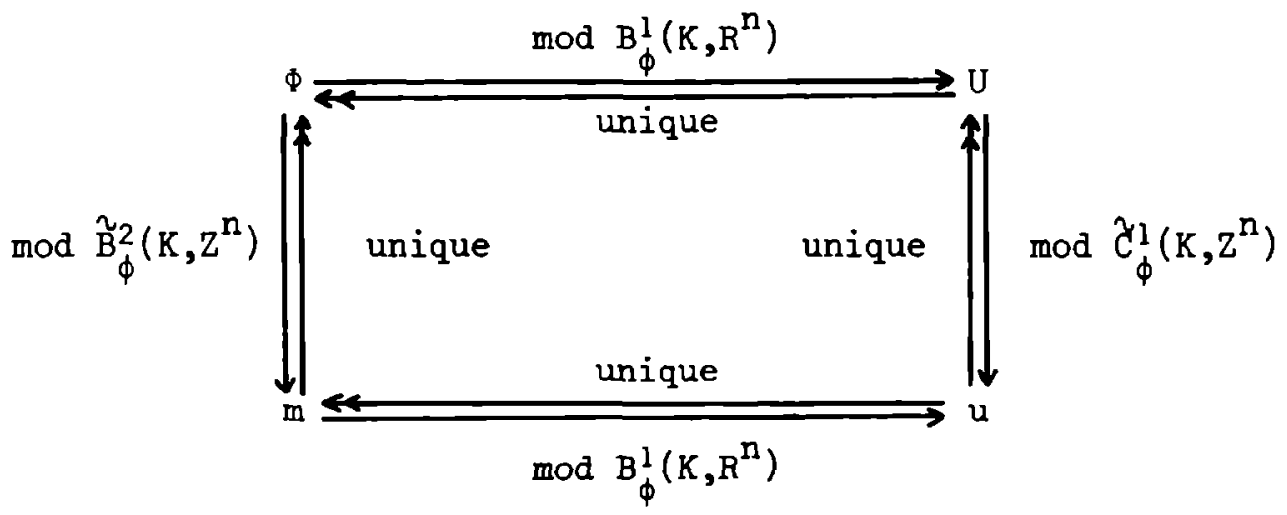


Let us finally remark that for $\Pi^{\prime}=P \Pi Q$ given as in (3.12), the torsion numbers of $\mathrm{H}_{\phi}^{2}\left(\mathrm{~K}, \mathrm{z}^{\mathrm{n}}\right)$ and of $\mathrm{H}_{\phi}^{1}\left(\mathrm{~K}, \mathrm{R}^{\mathrm{n}} / \mathrm{Z}^{\mathrm{n}}\right)$ then being $\mathrm{d}_{1}, \mathrm{~d}_{2}, \ldots, \mathrm{d}_{\mathrm{s}}$, one can always fix a given group $G$ of $(4.1)$ in such a way that the corresponding $m, \Phi$, $u$ and $U$ are as follows:

$$
\Phi=P^{-1} \Phi^{\prime} \text { with } \Phi^{\prime}=\left(\begin{array}{c}
\ell_{1} \\
\vdots \\
\ell_{s} \\
0 \\
\vdots \\
0
\end{array}\right) ; U=Q U^{\prime} \text { with } U^{\prime}=\left(\begin{array}{c}
\ell_{1} / d_{1} \\
\vdots \\
\ell_{s} / d_{s} \\
0 \\
\vdots \\
0
\end{array}\right)
$$

and

$$
\left(\begin{array}{ccc}
\ell_{1} \bmod & d_{l} \\
\vdots & \\
\ell_{s} & \bmod d_{s}
\end{array}\right) \Leftrightarrow[m] \in H_{\phi}^{2}\left(K, z^{n}\right) ;\left(\begin{array}{c}
\ell_{1} / d_{1} \\
\vdots \\
\ell_{s} / d_{s}
\end{array}\right) \Leftrightarrow\left[\pi_{*} u\right] \in H_{\phi}^{l}\left(K, R^{n} / z^{n}\right)
$$

for some $\left\{\ell_{1}, \ldots, \ell_{s} \in Z \mid 0 \leqslant \ell_{i}<d_{i}\right\}$. That $H_{\phi}^{2}\left(K, z^{n}\right) \cong H_{\phi}^{l}\left(K, R^{n} / Z^{n}\right)$ is in (5.28) manifest.

\section{Isomorphism}

i) General concepts.

If two space groups $G$ and $\bar{G}$ are isomorphic they may be obtained from extensions with the same $\phi(K)[1]$. So one can construct the following morphism of group extensions

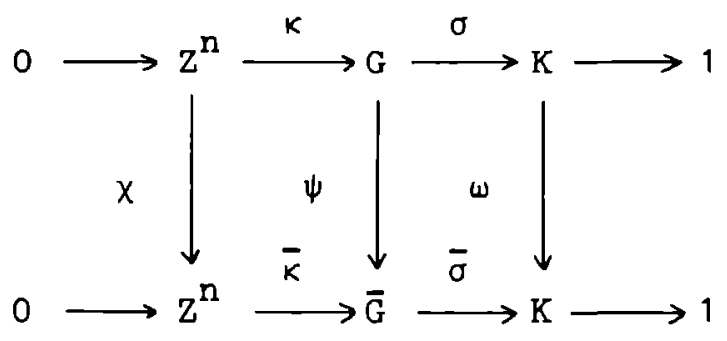

If $\psi$ is an isomorphism, so are $x=\psi / \mathrm{z}^{\mathrm{n}}$ and $\omega$. Without loss of generality 
one may suppose for $\psi[1, p .570]$ that

$$
\psi r(\alpha)=\bar{r}(\omega \alpha)
$$

From $r(\alpha)+\kappa a-r(\alpha)=\kappa \phi(\alpha)$ a follows $\bar{\kappa} \phi(\omega \alpha) \times a=\bar{r}(\omega \alpha)+\bar{k} \times a-\bar{r}(\omega \alpha)=$ $\bar{\kappa} \chi \phi(\alpha)$ a.

So

$$
\phi(\omega \alpha)=x \phi(\alpha) x^{-1}
$$

Hence, if $N$ is the normalizer of $\phi(K)$ in $G L(n, Z)$, then $x \in N$. In this way an homomorphic image of $N$ in $A u t(K)$ is defined:

$$
\xi: \mathrm{N} \rightarrow \operatorname{Aut}(\mathrm{K})
$$

by

$$
\xi_{X}=\omega \quad(x \in N, \omega \text { as fixed by }(6.3)) .
$$

One has

Ker $\xi=$ centralizer $z$ of $\phi(K)$ in $G L(n, Z)$.

Both $\mathrm{Z}$ and $\mathrm{N}$ are finitely generated $[5, \mathrm{p} .130]$ and of course $\mathrm{Z} 4 \mathrm{~N}$

$$
\begin{aligned}
& \mathrm{z}=\left\langle x_{1}, \ldots, x_{\lambda}\right\rangle \\
& \mathrm{N}=\left\langle x_{1}, \ldots, x_{\lambda}, \ldots, x_{\mu}\right\rangle
\end{aligned}
$$

ii) If $G$ and $\bar{G}(6.1)$ are given by their relation vectors $\Phi$ and $\bar{\Phi}$ respectively, one has according to $(6.2)$

$$
\Phi_{i}\left(r\left(\omega^{-1} \alpha_{1}\right), \ldots, r\left(\omega^{-1} \alpha_{v}\right)\right)=k x^{-1} \bar{g}_{i}
$$

We choose for each $\alpha \in K$ a fixed word $w_{\alpha}\left(\alpha_{1}, \ldots, \alpha_{v}\right)$ and we introduce new coset representatives $\tilde{r}(\alpha)=\kappa c(\alpha)+r(\alpha)$ such that 

1) $\tilde{r}\left(\alpha_{i}\right)=r\left(\alpha_{i}\right)$
$(i=1, \ldots, v)$
2) $\tilde{r}(\alpha)=w_{\alpha}\left(\tilde{r}\left(\alpha_{1}\right), \ldots, \tilde{r}\left(\alpha_{v}\right)\right)$,
$(\forall \alpha \in K)$

From (6.6) follows the relation

$$
\phi_{i}\left(\tilde{r}\left(\omega^{-1} \alpha_{1}\right), \ldots, \tilde{r}\left(\omega^{-1} \alpha_{v}\right)\right)=\kappa \chi^{-1} \vec{B}_{i}+\kappa \sum_{j=1}^{v} \pi_{i}\left(\omega^{-1} \alpha_{j}\right) \cdot c\left(\omega^{-1} \alpha_{j}\right)
$$

where $\pi_{i}\left(\omega^{-1} \alpha_{j}\right)$ is defined in a way analogous to (2.3).

Because

$$
\varepsilon=\phi_{i}\left(\omega^{-1} \alpha_{1}, \ldots, \omega^{-1} \alpha_{v}\right)=\pi \alpha_{j_{1}} \cdots \alpha_{j_{t}} \phi_{j}\left(\alpha_{1}, \ldots, \alpha_{v}\right) \alpha_{j_{t}}^{-1} \ldots \alpha_{j_{1}}^{-1}
$$

from (6.8) it follows that

$$
\begin{aligned}
\kappa^{-1} \phi_{i}\left(\tilde{r}\left(\omega^{-1} \alpha_{1}\right), \ldots, \tilde{r}\left(\omega^{-1} \alpha_{\nu}\right)\right) & =\sum_{j=1}^{v} \theta_{i j} k^{-1} \phi_{j}\left(\tilde{r}\left(\alpha_{1}\right), \ldots, \tilde{r}\left(\alpha_{\nu}\right)\right)= \\
& =\sum_{j=1}^{v} \theta_{i j} \tilde{B}_{j},
\end{aligned}
$$

for certain elements $\theta_{i j}$ of $2 K$ (compare with (5.9)). Hence

$$
x^{-1} \overline{\mathrm{g}}_{i}=\sum_{j=1}^{\nu} \theta_{i j} \tilde{\mathrm{g}}_{j}-\sum_{j=1}^{\nu} \pi_{i}\left(\omega^{-1} \alpha_{j}\right) \cdot c\left(\omega^{-1} \alpha_{j}\right)
$$

and because of $(6.7) \tilde{g}_{j}=g_{j}$, i.e.:

$$
\bar{g}_{i}=x \sum_{j=1}^{\nu} \theta_{i j} g_{j}-\sum_{j=1}^{\nu} \pi_{i}\left(\alpha_{j}\right) \cdot x c\left(\omega^{-1} \alpha_{j}\right) \text {. }
$$

Define

$$
Y=X \times\left(\begin{array}{ccc}
\theta_{11} & \cdots & \theta_{1 r} \\
\theta_{r 1} & \cdots & \theta_{r r}
\end{array}\right)=X \times \theta \quad \text { (direct product) }
$$

which is a $p \times p$ matrix. 
Then:

$$
\bar{\Phi} \sim \overline{\bar{\Phi}} \stackrel{\operatorname{def}}{=} Y \Phi
$$

\section{Proposition 4:}

An element $x \in N$ induces an autcrorphism $\lambda^{*}$ of the group $\mathrm{H}_{\phi}^{2}\left(K, \mathrm{z}^{\mathrm{n}}\right)$.

Proof:

a) Suppose $\bar{G}$ appears in a split extension: $\bar{\Phi} \sim 0$

$$
\bar{B}_{i}=\sum_{j=1}^{v} \pi_{i}\left(\alpha_{j}\right) \bar{c}\left(\alpha_{j}\right), \text { for } \bar{c} \in \mathbb{C}_{\phi}^{1}\left(K, z^{n}\right), \quad(i=1, \ldots, r)
$$

By (6.2) one obtains

$$
\Phi_{i}\left(r\left(\omega^{-1} \alpha_{1}\right), \ldots, r\left(\omega^{-1} \alpha_{\nu}\right)\right)=\kappa \sum_{j=1}^{\nu} \pi_{i}\left(\omega^{-1} \alpha_{j}\right) x^{-1} \bar{c}\left(\omega^{-1} \alpha_{j}\right)(i=1, \ldots, r)
$$

By choosing other representatives $r(\alpha)=k \chi^{-1} \bar{c}(\alpha)+\tilde{r}(\alpha)$ one has

$$
\Phi_{i}\left(\tilde{r}\left(\omega^{-1} \alpha_{1}\right), \ldots, \tilde{r}\left(\omega^{-1} \alpha_{\nu}\right)\right)=0
$$

Hence

and

$$
\Phi_{i}\left(\tilde{r}\left(\alpha_{1}\right), \ldots, \tilde{r}\left(\alpha_{\nu}\right)\right) \sim 0
$$

$$
\Phi \sim \tilde{\Phi} \sim 0
$$

If $\bar{\Phi}$ splits, so does $\Phi$ and vice versa.

b) If for given $\phi(K)$ all non-equivalent vectors $\{\phi\}$ are known, an element $x \in \mathbb{N}$ gives for every $\Phi$ a $\bar{\Phi}$ of an isomorphic group by (6.13). By construction $\overline{\bar{\phi}}$ is equivalent to one of the non-equivalent vectors $\{\Phi\}$. If $\phi_{1} \sim \phi_{2}$, then $\bar{\phi}_{1} \sim \overline{\bar{\phi}}_{2}$ because of a). Thus $x$ induces a permutation of the equivalence classes, which corresponds to an automorphism $\lambda^{*}$ of $\mathrm{H}_{\phi}^{2}\left(\mathrm{~K}, \mathrm{Z}^{\mathrm{n}}\right)$.

In this way one may construct the orbits of $x$ relative to $\mathrm{H}_{\phi}^{2}\left(\mathrm{~K}, \mathrm{z}^{\mathrm{n}}\right)$. As $N$ is finitely generated, the orbits of $N$ relative to $H_{\phi}^{2}\left(K, z^{n}\right)$ are determined by the orbits of the generators $x_{1}, \ldots, x_{\mu}$. The equivalence classes corresponding to one orbit of $N$ form an isomorphism class. 
For the practical calculation the following proposition is useful. Proposition 5:

An element of $\phi(K) \subseteq N$ induces the identity automorphism of $H_{\phi}^{2}\left(K, Z^{n}\right)$. Proof: Suppose $\rho \in \phi(K)$. Then from (6.2) one has

$$
\begin{aligned}
& \bar{k} \rho g_{i}=\phi_{i}\left(\bar{r}\left(\omega \alpha_{1}\right), \ldots, \bar{r}\left(\omega \alpha_{v}\right)\right) \\
& =\phi_{i}\left(\bar{x} c\left(\alpha_{1}\right)+\bar{r}(\rho)+\bar{r}\left(\alpha_{1}\right)-\bar{r}(\rho), \ldots, \bar{k} c\left(\alpha_{v}\right)+\bar{r}(\rho)+\right. \\
& \left.+\bar{r}\left(\alpha_{v}\right)-\bar{r}(\rho)\right) \\
& =\bar{\kappa} \sum_{j=1}^{\nu} \rho \pi_{i}\left(\alpha_{j}\right) \rho^{-1} \cdot c\left(\alpha_{j}\right)+\Phi_{i}\left(\bar{r}(\rho)+\bar{r}\left(\alpha_{1}\right)-\bar{r}(\rho), \ldots, \bar{r}(\rho)+\right. \\
& \left.+\bar{r}\left(\alpha_{\nu}\right)-\bar{r}(p)\right) \\
& =\bar{\kappa} \rho \sum_{j=1}^{\nu} \pi_{i}\left(\alpha_{j}\right) \rho^{-1} c\left(\alpha_{j}\right)+\bar{r}(\rho)+\Phi_{i}\left(\bar{r}\left(\alpha_{1}\right), \ldots, \bar{r}\left(\alpha_{\nu}\right)\right)-\bar{r}(\rho) .
\end{aligned}
$$

Hence

$$
g_{i}=\sum_{j=1}^{\nu} \pi_{i}\left(\alpha_{j}\right) \rho^{-1} c\left(\alpha_{j}\right)+\bar{g}_{i} \quad \text { for some } \quad c \in C_{\phi}^{l}\left(K, z^{n}\right)
$$

Then $\bar{\Phi}$ and $\Phi$ are equivalent.

iii) If $u$ and $\tilde{u}$ are systems of non-primitive translations of $G$ and $\bar{G}$ appearing in (6.1) one has [2], [5]

$$
\tilde{u}(\alpha) \equiv x u\left(\omega^{-1} \alpha\right)+(1-\alpha) f \quad\left(\bmod z^{n}\right) \quad(\forall \alpha \in K)
$$

Then $\bar{u}$ defined by

$$
\bar{u}(\alpha)=x u\left(\omega^{-1} \alpha\right) \quad(\forall \alpha \in K)
$$

is an equivalent system of non-primitive translations for $\bar{G}$.

The mapping $\mathrm{u}(\alpha) \longmapsto \overline{\mathrm{u}}(\alpha)=x \mathrm{u}\left(\omega^{-1} \alpha\right)$ induces an automorphism $\lambda^{*}$ of $\mathrm{H}_{\phi}^{\mathrm{l}}\left(\mathrm{K}, \mathrm{R}^{\mathrm{n}} / \mathrm{Z}^{\mathrm{n}}\right)$ determined by $x \in \mathbb{N}$. For a fixed choice $\omega^{-1} \alpha_{i}=\omega_{i}\left(\alpha_{1}, \ldots, \alpha_{v}\right)$ one may determine elements $\Omega_{i j}$ of $\mathrm{ZK}$ such that 


$$
u\left(\omega^{-1} \alpha_{i}\right) \equiv \sum_{j=1}^{v} \Omega_{i j} u\left(\alpha_{j}\right) \quad\left(\bmod z^{n}\right) \quad(i=1, \ldots, v)
$$

using $u(\alpha \beta) \equiv u(\alpha)+\alpha u(\beta) \quad\left(\bmod z^{n}\right)$.

Define the $q \times q$ matrix

$$
\mathrm{X}=x \times\left(\begin{array}{ccc}
\Omega_{11} & \ldots & \Omega_{1 v} \\
\vdots & & \\
\Omega_{v 1} & \ldots & \Omega_{v v}
\end{array}\right) \quad \text { (direct product) }
$$

Then in matrix form $(6.18)$ becomes

$$
\bar{U} \equiv X U \quad\left(\bmod z^{q}\right)
$$

so that going over to an equivalent vector $\overline{\bar{U}}$ one has

$$
\mathrm{U} \sim \overline{\bar{U}}=\mathrm{X} U
$$

Define

$$
X^{\prime}=Q^{-1} \times Q
$$

Then $(5.20)$ gives:

$$
\overline{\mathrm{U}^{\prime}}=\mathrm{X}^{\prime} \mathrm{U}^{\prime}
$$

By (6.23) one may determine the permutation of equivalence classes induced by $x \in N$.

iv) The relation between $X$ and $Y$.

These two matrices are not uniquely determined and neither is their mutual relationship. But without loss of generality we may place us in the situation (5.27) with $\Pi^{\prime}=P \Pi Q$ as in (3.12).

Define the $q \times p$ matrices 
and

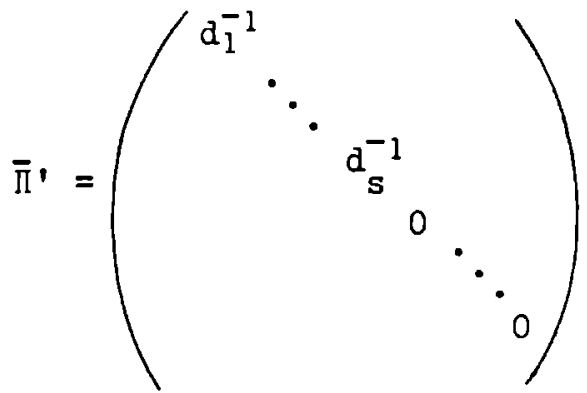

$$
\bar{\Pi}=Q \bar{\Pi} \cdot P
$$

Then using $(5.19),(6.22),(5.20)$, and noticing that $U^{\prime}=\bar{\pi} \cdot \Phi^{\prime}$ one has

$$
\overline{\bar{\phi}}=\Pi \overline{\bar{U}}=\Pi X U=\Pi X Q U^{\prime}=\pi X \bar{\Pi} \Phi=Y \Phi
$$

So for every $x \in \mathbb{N}$ one easily constructs the matrix $X$ and by using (6.26) one avoids the difficult word problem (6.11). One determines for each $\Phi$ a corresponding $\overline{\bar{\phi}}$ which must belong to one of the equivalence classes.

\section{Example}

In this section the techniques explained in the previous sections are displayed on a specific example. We choose here the point group $K=D_{2}$, generated by $\alpha, \beta,(\nu=2)$ and with defining relators $(r=3)$ :

$$
\Phi_{1}(\alpha, \beta)=\alpha^{2}, \Phi_{2}(\alpha, \beta)=\beta^{2}, \Phi_{3}(\alpha, \beta)=\alpha \beta \alpha \beta
$$

Hence the matrix $\pi$ of (3.2) is given by [see appendix]

$$
\Pi=\left(\begin{array}{ll}
N_{\alpha} & 0 \\
0 & N_{\beta} \\
N_{\alpha \beta} & N_{\alpha \beta}^{\alpha}
\end{array}\right)
$$

We now choose as $\phi(K)$ the 3-dimensional arithmetic point group 222 defined by

$$
\phi(\alpha)=\left(\begin{array}{lll}
-1 & -1 & \\
& -1 & 1
\end{array}\right), \quad \phi(\beta)=\left(\begin{array}{lll}
1 & & \\
& -1 & -1
\end{array}\right)
$$


Then we have:

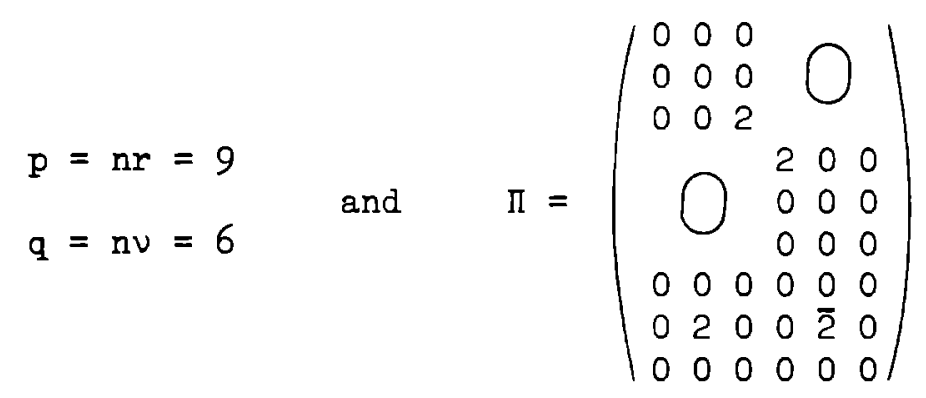

(we write $\bar{z}$ for $-z$ ).

The $\Pi^{\prime}$ of (3.12) becomes:

$$
\Pi \cdot=\left(\begin{array}{lll}
2 & & \\
2 & \\
2 & \\
& \\
& \\
& 0 \\
& 0 \\
& 0 \\
& 0 \\
& \\
& 0
\end{array}\right) \text { with } P=\left(\begin{array}{lllllllll}
1 & 0 & 0 & 1 & 0 & 0 & 0 & 0 & 0 \\
0 & 1 & 0 & 0 & 0 & 0 & 0 & 1 & 0 \\
0 & 0 & 1 & 0 & 0 & 0 & 0 & 0 & 0 \\
1 & 0 & 0 & 0 & 0 & 0 & 0 & 0 & 0 \\
0 & 0 & 0 & 0 & 1 & 0 & 0 & 0 & 0 \\
0 & 0 & 0 & 0 & 0 & 1 & 0 & 0 & 0 \\
0 & 0 & 0 & 0 & 0 & 0 & 1 & 0 & 0 \\
0 & 1 & 0 & 0 & 0 & 0 & 0 & 0 & 0 \\
0 & 0 & 0 & 0 & 0 & 0 & 0 & 0 & 1
\end{array}\right) \text { and } Q=\left(\begin{array}{llllll}
1 & 0 & 0 & 1 & 0 & 0 \\
0 & 1 & 0 & 0 & 1 & 0 \\
0 & 0 & 1 & 0 & 0 & 0 \\
1 & 0 & 0 & 0 & 0 & 0 \\
0 & 0 & 0 & 0 & 1 & 0 \\
0 & 0 & 0 & 0 & 0 & 1
\end{array}\right)
$$

So

$$
\mathrm{H}_{\phi}^{2}\left(\mathrm{D}_{2}, \mathrm{Z}^{3}\right) \stackrel{\simeq}{=} \mathrm{C}_{2} \times \mathrm{C}_{2} \times \mathrm{C}_{2}
$$

There are 8 non-equivalent extensions. They can be characterized by $\left(\ell_{1} \ell_{2} \ell_{3}\right)$ with $\ell_{i}=0$ or $1 \quad(i=1,2,3)$, as the general form of an element of:

$$
\mathrm{H}_{\phi}^{2}\left(D_{2}, \mathrm{Z}^{3}\right)=\left\langle\mathrm{e}_{1}, \mathrm{e}_{2}, \mathrm{e}_{3} ; 2 \mathrm{e}_{1}=2 \mathrm{e}_{2}=2 \mathrm{e}_{3}=0\right\rangle
$$

is

$$
\ell_{1} e_{1}+\ell_{2} e_{2}+\ell_{3} e_{3} .
$$

According to (5.27) we have correspondingly 


$$
\begin{aligned}
& \Phi^{\prime}=\left(\begin{array}{l}
\ell_{1} \\
\ell_{2} \\
\ell_{3} \\
0 \\
1 \\
0
\end{array}\right) \quad \text { and } \Phi=P^{-1} \Phi^{\prime}=\left(\begin{array}{l}
0 \\
0 \\
\ell_{3} \\
\ell_{1} \\
0 \\
0 \\
0 \\
\ell_{2} \\
0
\end{array}\right) \\
& U^{\prime}=\left(\begin{array}{c}
\ell_{1} / 2 \\
\ell_{2} / 2 \\
\ell_{3} / 2 \\
0 \\
1 \\
0
\end{array}\right) \quad \text { and } \quad U=Q U^{\prime}=\left(\begin{array}{c}
\ell_{1} / 2 \\
\ell_{2} / 2 \\
\ell_{3} / 2 \\
\ell_{1} / 2 \\
0 \\
0
\end{array}\right)
\end{aligned}
$$

One verifies that $\Phi$ and $U$ satisfy $(5.19)$ and that (after identification of $\mathrm{H}_{\phi}^{2}\left(\mathrm{D}_{2}, \mathrm{Z}^{3}\right)$ with its isomorphic image $\left.(7.6)\right)$

$\Phi=\ell_{1} e_{1}+\ell_{2} e_{2}+\ell_{3} e_{3}$ where according to $(3.13) e_{i}=P^{-1} \varepsilon_{i}(i=1,2,3)$.

If we now denote by $a_{1}, a_{2}, a_{3}$ the image under $k$ of the free generators $z_{i}$ of $z^{3}:$

$$
a_{i}=k z_{i} \quad \text { where } z_{1}=\left(\begin{array}{l}
1 \\
0 \\
0
\end{array}\right), z_{2}=\left(\begin{array}{l}
0 \\
1 \\
0
\end{array}\right), z_{3}=\left(\begin{array}{l}
0 \\
0 \\
1
\end{array}\right) \text {, }
$$

and

$$
\mathrm{e}_{1}=\left(\begin{array}{l}
0 \\
z_{1} \\
0
\end{array}\right), \mathrm{e}_{2}=\left(\begin{array}{l}
0 \\
0 \\
z_{2}
\end{array}\right), \mathrm{e}_{3}=\left(\begin{array}{l}
\mathrm{z}_{3} \\
0 \\
0
\end{array}\right) \text {, then (7.8) and (7.9) }
$$

mean that for the groups $G$ of (6.1) corresponding to the inequivalent group extensions $\left(\ell_{1} \ell_{2} \ell_{3}\right)$ one has $(\operatorname{see}(3.1))$ :

$$
\begin{aligned}
& \Phi_{1}(r(\alpha), r(\beta))=2 r(\alpha)=\ell_{3} a_{3} \\
& \Phi_{2}(r(\alpha), r(\beta))=2 r(\beta)=\ell_{1} a_{1} \\
& \Phi_{3}(r(\alpha), r(\beta))=2(r(\alpha)+r(\beta))=\ell_{2} a_{2}
\end{aligned}
$$


and $(4.10)$ :

$$
\begin{aligned}
& u(\alpha)=\frac{\ell_{1}}{2} z_{1}+\frac{\ell_{2}}{2} z_{2}+\frac{\ell_{3}}{2} z_{3} \\
& u(\beta)=\frac{\ell_{1}}{2} z_{1} \\
& u(\alpha \beta)=(u(\alpha)+\alpha u(\beta))=\frac{\ell_{2}}{2} z_{2}+\frac{\ell_{3}}{2} z_{3} \quad \text { and of course } \\
& u(\varepsilon)=0,
\end{aligned}
$$

where we extended $U$ by (5.25) to a system of non-primitive translations $u$, putting $c(\alpha)=0$.

The corresponding factor system is then determined according to $(5.26)$ :

$$
\begin{aligned}
& \mathrm{m}(\varepsilon, \varepsilon)=\mathrm{m}(\varepsilon, \alpha)=\ldots+\mathrm{m}(\alpha, \varepsilon)=0 \quad \text { (normed factor set) } \\
& \mathrm{m}(\alpha, \alpha)=\ell_{3} z_{3} ; \mathrm{m}(\beta, \alpha)=\ell_{1} z_{1}-\ell_{2} z_{2}-\ell_{3} z_{3} ; m(\alpha \beta, \alpha)=-\ell_{1} z_{1}+\ell_{2} z_{2} \\
& m(\alpha, \beta)=0 ; ; m(\beta, \beta)=\ell_{1} z_{1} \quad ; m(\alpha \beta, \beta)=-\ell_{1} z_{1} \\
& m(\alpha, \alpha \beta)=\ell_{3} z_{3} ; m(\beta, \alpha \beta)=-\ell_{2} z_{2}-\ell_{3} z_{3} ; m(\alpha \beta, \alpha \beta)=\ell_{2} z_{2}
\end{aligned}
$$

One verifies that (7.12) fulfil (7.10). This factor set can also be obtained directly from (7.10) after calculation of the $\gamma_{i}(\alpha, \beta)$ of (5.6) The result is: (omitting the trivial elements)

$$
\begin{array}{ll}
m(\alpha, \alpha)=g_{1} ; m(\beta, \alpha)=\beta g_{1}+g_{2}-g_{3} ; m(\alpha \beta, \alpha)=-g_{2}+g_{3} \\
m(\alpha, \beta)=0 ; m(\beta, \beta)=g_{2} ; m(\alpha \beta, \beta)=\alpha g_{2} \\
m(\alpha, \alpha \beta)=g_{1} ; m(\beta, \alpha \beta)=-g_{1}+\beta g_{3} ; m(\alpha \beta, \alpha \beta)=g_{3}
\end{array}
$$

where according to $(7.10)$

$$
g_{1}=\ell_{3} z_{3}, g_{2}=\ell_{1} z_{1} \text { and } g_{3}=\ell_{2} z_{2} \text {. }
$$


By choosing $U$ in the standard form (5.27) and by determining $u$ from $U$ by (5.24) putting $c(\alpha)=0$ one has removed two degrees of freedom. With the same $\Phi$ one may add to each vector $U$ the vector

$$
V=Q V^{\prime}=Q\left(\begin{array}{l}
0 \\
0 \\
0 \\
r_{1} \\
r_{2} \\
r_{3}
\end{array}\right)=\left(\begin{array}{c}
-r_{1} \\
r_{2} \\
0 \\
0 \\
r_{2} \\
r_{3}
\end{array}\right) \quad r_{1}, r_{2}, r_{3} \in R
$$

With the same vector $U$ one may choose $u^{\prime}(\alpha \beta)=u(\alpha \beta)+i c(\alpha \beta)$ for

$$
c(\alpha \beta)=\left(\begin{array}{l}
c_{1} \\
c_{2} \\
c_{3}
\end{array}\right) \quad \in z^{3}
$$

Therefore without changing $\Phi$ one may add to the factor system (7.12) the following one:

$$
\begin{array}{lll}
m^{\prime}(\alpha, \alpha)=0 & m^{\prime}(\beta, \alpha)=-c(\alpha \beta) & m^{\prime}(\alpha \beta, \alpha)=c(\alpha \beta) \\
m^{\prime}(\alpha, \beta)=-c(\alpha \beta) & m^{\prime}(\beta, \beta)=0 & m^{\prime}(\alpha \beta, \beta)=c(\alpha \beta) \\
m^{\prime}(\alpha, \alpha \beta)=\alpha c(\alpha \beta) & m^{\prime}(\beta, \alpha \beta)=\beta c(\alpha \beta) & m^{\prime}(\alpha \beta, \alpha \beta)=2 c_{2} z_{2}
\end{array}
$$

which is an element of $\tilde{B}_{\phi}^{2}\left(K, z^{3}\right)$.

We now consider the isomorphism classes of these non-equivalent extensions. Putting

$$
\begin{array}{ll}
x_{1}=\left(\begin{array}{ccc}
-1 & -1 & \\
& -1 & 1
\end{array}\right)=\alpha \quad x_{2}=\left(\begin{array}{ccc}
1 & & \\
& -1 & -1
\end{array}\right)=\beta \quad x_{3}=\left(\begin{array}{lll}
1 & 1 & \\
& 1 & -1
\end{array}\right) \\
x_{4}=\left(\begin{array}{lll}
1 & 0 & 1 \\
& 0 & 0
\end{array}\right) \quad \text { and } & x_{5}=\left(\begin{array}{lll}
0 & 1 & 0 \\
0 & 0 & 1 \\
1 & 0 & 0
\end{array}\right)
\end{array}
$$


one has

$$
\begin{aligned}
& \phi(K)=\left\langle x_{1}, x_{2}\right\rangle \quad \text { arithmetic point group } \\
& Z=\left\langle x_{1}, x_{2}, x_{3}\right\rangle \text { centralizer of } \phi(K) \text { in } G L(3, Z) \\
& N=\left\langle x_{1}, \ldots, x_{5}\right\rangle \text { normalizer of } \phi(K\rangle \text { in } G L(3, Z) .
\end{aligned}
$$

The automorphisms $\omega_{i}$ of $(6.1)$ induced by $x_{i}$ respectively and determined according to $(6.3)$ are:

$$
\begin{aligned}
& \omega_{1}=\omega_{2}=\omega_{3}=\text { identity automorphism of } K \text { (because } x_{1}, x_{2}, x_{3} \in \mathrm{Z} \text { ) } \\
& \omega_{4}: \alpha \rightarrow \alpha \beta \rightarrow \alpha, \beta \rightarrow \beta \\
& \omega_{5}: \alpha \rightarrow \alpha \beta \rightarrow \beta \rightarrow \alpha
\end{aligned}
$$

We indicate below the calculation of the orbits of $\mathrm{H}_{\phi}^{2}\left(\mathrm{D}_{2}, \mathrm{Z}^{3}\right)$ relative to $x_{4}$ and quote the corresponding results for the cases of $x_{3}$ and $x_{5}$. Using (6.11) and (6.12) one obtains

$$
\theta_{4}=\left(\begin{array}{ccc}
0 & 0 & 1 \\
0 & 1 & 0 \\
1 & (1+\alpha) & 0
\end{array}\right) \quad \text { and } \quad Y_{4}=\left(\begin{array}{ccc}
0 & 0 & x_{4} \\
0 & x_{4} & 0 \\
x_{4} & x_{4}(1+\alpha) & 0
\end{array}\right)
$$

This gives with $(6.13)$ and $(7.8)$ :

$$
\bar{\Phi}^{\prime}(4)=P Y_{4} P^{-1} \Phi^{\prime}=\left(\begin{array}{l}
\ell_{1} \\
\ell_{3} \\
\ell_{2} \\
0 \\
\vdots \\
0
\end{array}\right)=\bar{\Phi}(4)
$$

Numbering the elements $\left(\ell_{1} \ell_{2} \ell_{3}\right)$ of $\mathrm{H}_{\phi}^{2}\left(\mathrm{D}_{2}, \mathrm{Z}^{3}\right)$ according to 


$$
\begin{aligned}
& 1 \equiv(000), 2 \equiv(100), 3 \equiv(010), 4 \equiv(001), 5 \equiv(110), 6 \equiv(101), \\
& 7 \equiv(011), 8 \equiv(111)
\end{aligned}
$$

the induced automorphism $\lambda_{4}^{*}:\left(\ell_{1} \ell_{2} \ell_{3}\right) \rightarrow\left(\ell_{1} \ell_{3} \ell_{2}\right)$ is given by the following permutation

$$
\lambda_{4}^{*}:(1)(2)(34)(56)(7)(8)
$$

The same result may be obtained from (6.22) using (6.19):

$$
\Omega_{4}=\left(\begin{array}{ll}
1 & \alpha \\
0 & 1
\end{array}\right) \quad \text { and } \quad x_{4}=\left(\begin{array}{cc}
x_{4} & x_{4}{ }^{\alpha} \\
& \\
0 & x_{4}
\end{array}\right)
$$

Then

$$
\overline{\bar{U}}_{(4)}=Q^{-1} \mathrm{X}_{4} Q U^{\prime}=\left(\begin{array}{c}
\ell_{1 / 2} \\
l_{3 / 2} \\
l_{2 / 2} \\
l_{1 / 2} \\
0 \\
0
\end{array}\right) \sim\left(\begin{array}{c}
l_{1 / 2} \\
\ell_{3 / 2} \\
\ell_{2 / 2} \\
0 \\
0 \\
0
\end{array}\right)=\bar{U}(4)
$$

giving rise as it should to the same permutation. One verifies that with $\overline{\mathrm{I}}$ of $(6.25)$ one has

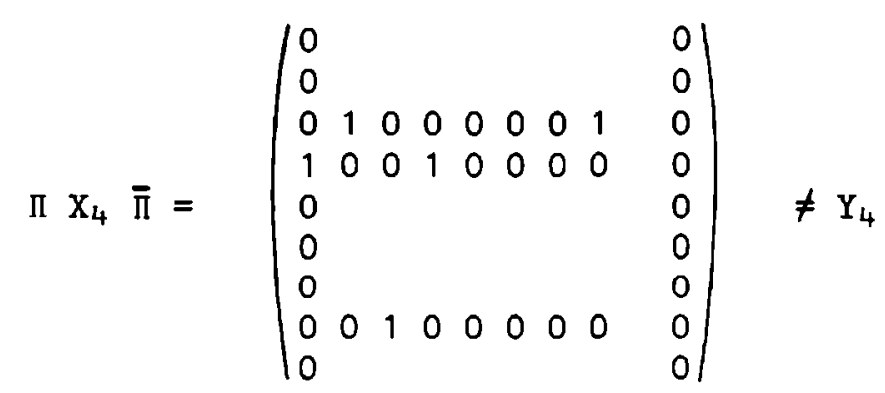

and indeed the relation $\pi X_{4} \bar{\Pi} \Phi=Y_{4} \Phi$ is satisfied.

In the same way one finds that $\lambda_{3}^{*}$ is the identity permutation, as are of course $\lambda_{1}^{*}$ and $\lambda_{2}^{*}$. For $x_{5}$ one finds: 


$$
\theta_{5}=\left(\begin{array}{ccc}
0 & 1 & 0 \\
0 & 0 & 1 \\
\beta & (1+\alpha \beta) & 0
\end{array}\right) \quad \text { and } \quad Y_{5}=\left(\begin{array}{ccc}
0 & x_{5} & 0 \\
0 & 0 & x_{5} \\
x_{5} \beta & x_{5}(1+\alpha \beta) & 0
\end{array}\right)
$$

The induced $\lambda_{5}^{*}$ is given by $\left(\ell_{1} \ell_{2} \ell_{3}\right) \mapsto\left(\ell_{2}, \ell_{3}, \ell_{1}\right)$.

Therefore we have

$$
\begin{aligned}
& \lambda_{5}^{*}:(1)\left(\begin{array}{lll}
2 & 3 & 4
\end{array}\right)\left(\begin{array}{lll}
5 & 6 & 7
\end{array}\right)(8) \\
& \Omega_{5}:=\left(\begin{array}{ll}
0 & 1 \\
1 & \alpha
\end{array}\right) \quad \text { and } \quad x_{5}=\left(\begin{array}{ll}
0 & x_{5} \\
& \\
x_{5} & x_{5} a
\end{array}\right) .
\end{aligned}
$$

This gives the same permutation.

From (7.19) and (7.22) one sees that the orbits of $\mathrm{H}_{\phi}^{2}\left(D_{2}, Z^{3}\right)$ relative to $N$ are

$$
\text { (1) }\left(\begin{array}{lll}
2 & 3 & 4
\end{array}\right)\left(\begin{array}{lll}
5 & 6 & 7
\end{array}\right) \text { and }(8)
$$

These are also the four isomorphism classes: the first containing the equivalence class 1, the second the classes 2,3 and 4 , the third the classes 5,6 and 7 and the fourth class 8. Hence for the arithmetic point group 222 one has 4 non-isomorphic 3-dimensional space groups denoted in the international notation [10] by $\mathrm{P} 222, \mathrm{P} 2{ }_{1} 22, \mathrm{P} 2{ }_{1} 2_{1} 2$ and $\mathrm{P} 2{ }_{1}{ }^{2}{ }_{1}{ }_{1}$.

They are given by the following table (the relations below are of course only the non-common part of the defining relations (3.1)).

\begin{tabular}{|l|c|c|c|c|}
\hline Non-isomorphic space groups & P222 & P2 ${ }_{1} 22$ & $P 2_{1}{ }_{1} 2$ & $P 2_{1}{ }_{1}{ }_{1}$ \\
\hline$g_{1}=2 r(\alpha)$ & 0 & 0 & 0 & $a_{3}$ \\
$g_{2}=2 r(\beta)$ & 0 & $a_{1}$ & $a_{1}$ & $a_{1}$ \\
$g_{3}=2(r(\alpha)+r(\beta))$ & 0 & 0 & $a_{2}$ & $a_{2}$ \\
\hline$K u(\alpha)$ & 0 & $\frac{1}{2} a_{1}$ & $\frac{1}{2}\left(a_{1}+a_{2}\right)$ & $\frac{1}{2}\left(a_{1}+a_{2}+a_{3}\right)$ \\
$\kappa u(\beta)$ & 0 & $\frac{1}{2} a_{1}$ & $\frac{1}{2} a_{1}$ & $\frac{1}{2} a_{1}$ \\
$\kappa u(\alpha \beta)$ & 0 & 0 & $\frac{1}{2} a_{2}$ & $\frac{1}{2}\left(a_{2}+a_{3}\right)$ \\
& & & & \\
\hline
\end{tabular}


The group P222 is the split extension of $Z^{3}$ and 222 and is called a symrorphic space group.

\section{Conclusion}

In general for an extension of an abelian group $A$ by a finite group $K$ one needs the extension conditions for this $K$. For generalized magnetic point groups up to the dimension four these extension conditions are given in the appendix.

For an extension of $\mathrm{z}^{\mathrm{n}}$ by a finite group $\mathrm{K}$ these conditions are not explicitly needed. Already the elements $\pi_{i}\left(\alpha_{j}\right)(2.2)$ derived from the defining relations of $K$ and the monomorphism $\phi: K \rightarrow G L(n, Z)$ provide equations to determine all non-equivalent extensions, i.e. to determine $\mathrm{H}_{\phi}^{2}\left(K, \mathrm{z}^{\mathrm{n}}\right)$. Because of the isomorphism $\mathrm{H}_{\phi}^{2}\left(\mathrm{~K}, \mathrm{z}^{\mathrm{n}}\right) \stackrel{\cong}{=} \mathrm{H}_{\phi}^{1}\left(\mathrm{~K}, \mathrm{R}^{\mathrm{n}} / \mathrm{Z}^{\mathrm{n}}\right)$ non-equivalent extensions may also be determined by corresponding systems of non-primitive translations.

The groups $H_{\phi}^{2}\left(K, z^{n}\right)$ and $H_{\phi}^{1}\left(K, R^{n} / z^{n}\right)$ have been determined for all $(3+1)-$ reducible four-dimensional arithmetic crystal classes. The results are published elsewhere [6]. 
Extension conditions for groups isomorphic to four-dimensional GM point groups

The four-dimensional GM point groups belong to the following isomorphism classes:

$$
\begin{array}{ll}
C_{n}(n=1,2,3,4,6) & =\left\langle\alpha ; \alpha^{n}=1\right\rangle \\
D_{n}(n=2,3,4,6) & =\left\langle\alpha, \beta ; \alpha^{n}=\beta^{2}=(\alpha \beta)^{2}=1\right\rangle \\
T & =\left\langle\alpha, \beta ; \alpha^{3}=\beta^{2}=(\alpha \beta)^{3}=1\right\rangle \\
0 & =\left\langle\alpha, \beta ; \alpha^{4}=\beta^{3}=(\alpha \beta)^{2}=1\right\rangle
\end{array}
$$

and the direct products $\mathrm{C}_{\mathrm{n}} \times \mathrm{C}_{2}, \mathrm{C}_{\mathrm{n}} \times \mathrm{C}_{2} \times \mathrm{C}_{2}, \mathrm{D}_{\mathrm{n}} \times \mathrm{C}_{2}, \mathrm{D}_{\mathrm{n}} \times \mathrm{C}_{2} \times \mathrm{C}_{2}, \mathrm{~T} \times \mathrm{C}_{2}$, $\mathrm{T} \times \mathrm{C}_{2} \times \mathrm{C}_{2}, \mathrm{O} \times \mathrm{C}_{2}, \mathrm{O} \times \mathrm{C}_{2} \times \mathrm{C}_{2}$.

Notice that $C_{3} \times C_{2} \cong C_{6} ; D_{3} \times C_{2} \cong D_{6}$ and $D_{2} \cong C_{2} \times C_{2}$.

In the following table for a group $G \times C_{2} \times C_{2}$, where $G=\left\langle\alpha, \beta ; \alpha^{n}=\beta^{l}=\right.$ $(\alpha \beta)^{\mathrm{m}}=1>$, are given: 1) the defining relations $\phi_{i}(\alpha, \beta, \gamma, \delta)=1$

$$
(i=1, \ldots, 10) \text {; }
$$

2) a set of generating elements for the $\mathrm{ZK}$-module formed by the solutions of (2.5); each of these extension conditions has 10 components;

3) the elements $\pi_{i}\left(\alpha_{j}\right)$ in (2.3) (i=1,..,10; $j=1, \ldots, 4)$.

The information for the groups $\mathrm{D}_{\mathrm{n}} \times \mathrm{C}_{2} \times \mathrm{C}_{2}, \mathrm{~T} \times \mathrm{C}_{2} \times \mathrm{C}_{2}$ and $\mathrm{O} \times \mathrm{C}_{2} \times \mathrm{C}_{2}$ is obtained from the table by taking $(n, l, m)=(n, 2,2),(3,2,3)$ and $(4,3,2)$ respectively. The information for other groups, which are subgroups of these three groups, may be obtained by restriction to those values of $i$ for which $\phi_{i}(\alpha, \beta, \gamma, \delta)=1$ is a relation of the smaller group and to those extension conditions which have entries only for these values of $i$. The corresponding rows and columns in table II are indicated in table I. 
Notice that in table II the elements $\pi_{i}\left(\alpha_{j}\right)$ are in transposed order relative to definition (3.2).

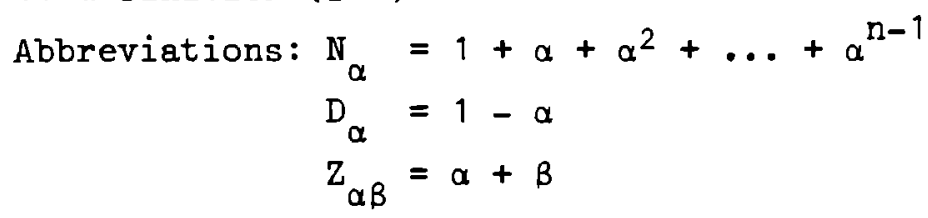

For a group $\mathrm{K} \times \mathrm{C}_{2} \times \mathrm{C}_{2} \mathrm{~K}_{\alpha}$ is an element of the group ring $\mathrm{ZK}$ such that $\mathrm{K}_{\alpha} \mathbb{N}_{\alpha}=\sum_{\zeta \in \mathrm{K}} \zeta$. The words representing $\mathrm{K}_{\alpha}$ may be different in different groups:

\begin{tabular}{|c|c|c|c|}
\hline & $\mathrm{K}^{2} \mathrm{D}_{\mathrm{n}}$ & $\mathrm{K}=\mathrm{T}$ & $\mathrm{K}=0$ \\
\hline $\mathrm{K}_{\alpha}$ & $\mathrm{N}_{\beta}$ & $\mathrm{N}_{\beta}(1+\alpha \beta)$ & $\mathrm{N}_{\beta}\left(1+\alpha \beta^{2}\right)$ \\
$\mathrm{K}_{\beta}$ & $\mathrm{N}_{\alpha}$ & $\mathrm{N}_{\alpha}(1+\beta \alpha)$ & $\mathrm{N}_{\alpha}\left(1+\beta^{2} \alpha\right)$ \\
$\mathrm{K}_{\alpha \beta}$ & $\mathrm{N}_{\alpha}$ & $\mathrm{N}_{\beta}(1+\alpha)$ & $\mathrm{N}_{\beta}(1+\alpha)\left(1+\alpha \beta^{2}\right)$ \\
\hline
\end{tabular}

\begin{tabular}{|l|l|l|l|l|}
\hline Group & relations $\Phi_{i}$ & generators & $\mathrm{n}, \ell, \mathrm{m}$ & $\begin{array}{l}\text { elements of the } \\
\text { ZK-module (rows) }\end{array}$ \\
\hline $\mathrm{C}_{\mathrm{n}}$ & $\mathrm{i}=1$ & & & \\
$\mathrm{C}_{\mathrm{n}} \times \mathrm{C}_{2}$ & $\mathrm{i}=1,4,5$ & $\alpha$ & $\mathrm{n}, 0,0$ & $\mathrm{H}_{1}$ \\
$\mathrm{C}_{\mathrm{n}} \times \mathrm{C}_{2} \times \mathrm{C}_{2}$ & $\mathrm{i}=1,4,5,7,8,10$ & $\alpha, \gamma, \delta$ & $\mathrm{n}, 0,0$ & $\mathrm{H}_{1}, \mathrm{H}_{5}, \mathrm{H}_{6}, \mathrm{H}_{7}$ \\
& & & $\mathrm{n}, 0,0$ & $\mathrm{H}_{1}, \mathrm{H}_{5}, \mathrm{H}_{6}, \mathrm{H}_{7}, \mathrm{H}_{11}, \mathrm{H}_{12}$ \\
$\mathrm{D}_{\mathrm{n}}$ & $\mathrm{i}=1,2,3$ & $\alpha, \beta$ & $\mathrm{n}, 2,2$ & $\mathrm{H}_{13}, \mathrm{H}_{16}, \mathrm{H}_{17}, \mathrm{H}_{3}, \mathrm{H}_{4}$ \\
$\mathrm{D}_{\mathrm{n}} \times \mathrm{C}_{2}$ & $i=1,2, \ldots, 6$ & $\alpha, \beta, \gamma$ & $\mathrm{n}, 2,2$ & $\mathrm{H}_{1}, \mathrm{H}_{2}, \ldots, \mathrm{H}_{10}$ \\
$\mathrm{~T}$ & $\mathrm{i}=1,2,3$ & $\alpha, \beta$ & $3,2,3$ & $\mathrm{H}_{1}, \ldots, \mathrm{H}_{4}$ \\
$\mathrm{~T} \times \mathrm{C}_{2}$ & $\mathrm{i}=1,2, \ldots, 6$ & $\alpha, \beta, \gamma$ & $3,2,3$ & $\mathrm{H}_{1}, \ldots, \mathrm{H}_{10}$ \\
0 & $\mathrm{i}=1,2,3$ & $\alpha, \beta$ & $4,3,2$ & $\mathrm{H}_{1}, \ldots, \mathrm{H}_{4}$ \\
$0 \times \mathrm{C}_{2}$ & $\mathrm{i}=1,2, \ldots, 6$ & $\alpha, \beta, \gamma$ & $4,3,2$ & $\mathrm{H}_{1}, \ldots, \mathrm{H}_{10}$ \\
& & & \\
\hline
\end{tabular}




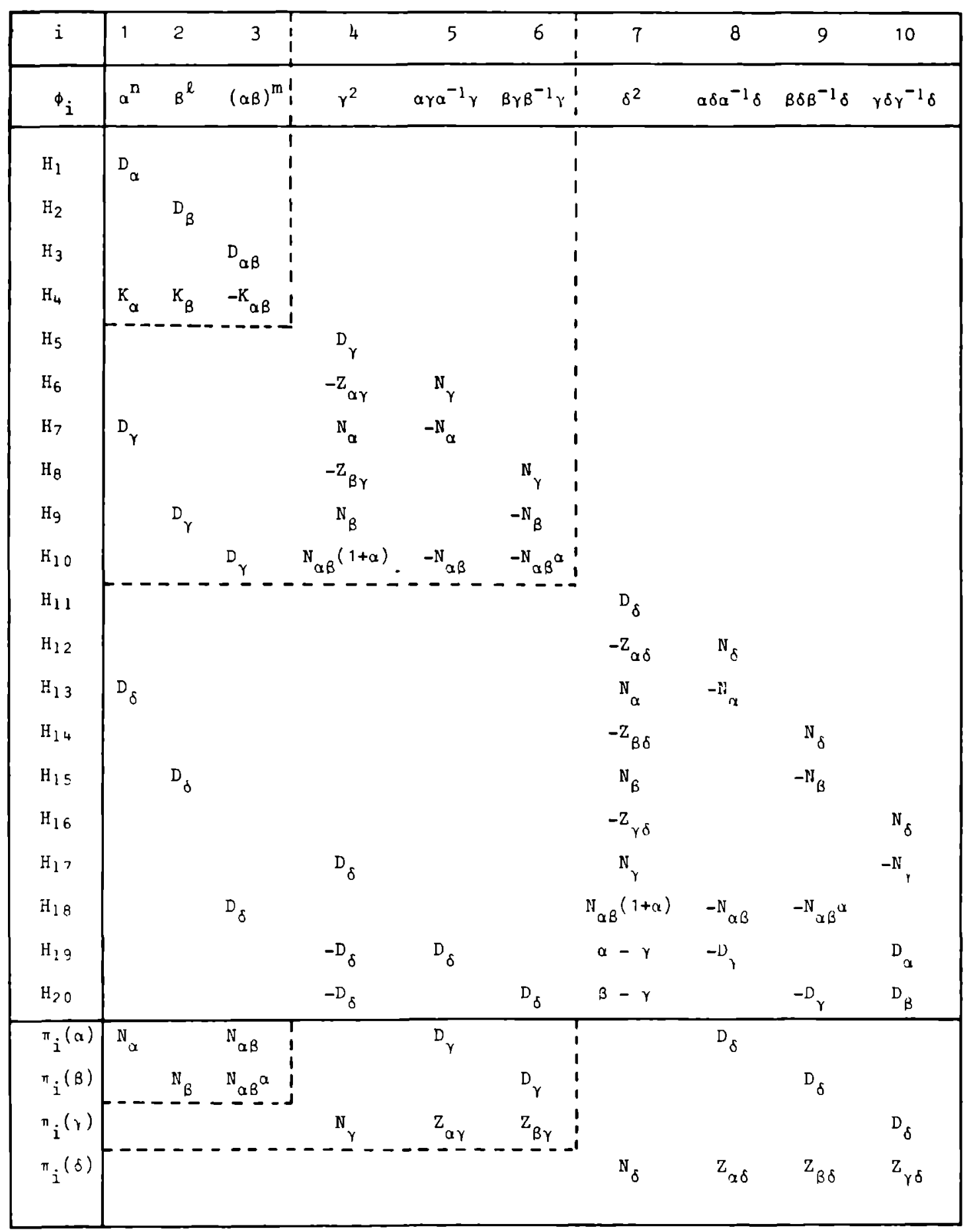




\section{PART THREE: FOUR-DIMENSIONAL EUCLIDEAN CRYSTAL CLASSES CORRESPONDING TO GM POINT GROUPS}

1. Introduction

A n-dimensional space group $G^{\mathrm{n}}$ may be obtained [1] from an extension $0 \longrightarrow \mathrm{z}^{\mathrm{n}} \longrightarrow \mathrm{G}^{\mathrm{n}} \longrightarrow \mathrm{K} \longrightarrow 1$

of a free abelian group of rank $n$ by a finite group $K$ with a monomorphism $\phi: K \rightarrow G L(n, Z)$. To obtain all non-isomorphic space groups it is sufficient to consider one representative $\phi(K)$ of each arithmetic crystal class. Elsewhere [2] a method has been given to determine all non-isomorphic extensions (1.1) once $\phi(K)$ is given. So the remaining problem is the determination of the arithmetic crystal classes. We limit ourselves here to those point.groups which are R-reducible into a (n-1)-dimensional and a one-dimensional part. (F-reducibility means full reducibility in $G L(n, F)$ for $F$ a ring).

We call these groups generalized magnetic point groups (GM point groups). The reason of this limitation is that the space groups (1.1) found for these crystal classes are isomorphic to discrete subgroups of the inhomogeneous Lorentz group. Actually each relativistic space-time group with a finite point group is isomorphic to a space group $G^{n}(1.1)$ with $\phi(K)$ a $G M$ point group [3].

For $n=4$ the problem of the determination of all arithmetic crystal classes has been treated by Zassenhaus [4] and by Bülow [5] who used a grouptheoretical computer program of Neubüser. Both used an algebraic method taking account of the work by Dade [6] who determined the maximal finite groups of $4 \times 4$ integral matrices. The method used here to determine the four-dimensional arithmetic crystal classes corresponding to a GM point group is rather a geometric one.

In section 2 we define a GM point group and show the one-to-one correspondence between $n$-dimensional GM point groups and $(n-1)$-dimensional magnetic point groups. For a fixed choice of the 1-dimensional subspace left invariant, the GM point groups are in one-to-one correspondence with Euclidean point 
groups. For the geometrically non-equivalent ones among these Euclidean point groups in section 3 a construction is given of the lattices left invariant. Expressing these point groups with respect to bases of these invariant lattices one obtains the arithmetic point groups. In section 4 the four-dimensional arithmetic crystal classes corresponding to GM point groups are determined.

In an appendix are given the four-dimensional geometric and arithmetic crystal classes corresponding to a GM point group and the four-dimensional Bravais classes of the lattices having a GM point group as holohedry. The four-dimensional geometric classes have already been published earlier by Hurley $[7,8,9]$. His $88(3+1)$-reducible classes are in agreement with our determination. The Bravais classes have been published by Mackay and Pawley [10] - They do not give a systematic derivation and their list is not complete. We have checked our 412 arithmetic crystal classes by the list of Neubüser and Bülow. After correcting some errors and omissions, our list agrees with theirs, according to a preliminary comparison.

\section{GM point groups}

Consider the product space of a n-dimensional Euclidean space by a onedimensional one. The subgroup of all linear $n+1$-dimensional transformations which leave the metric in both spaces invariant is the homogeneous pseudoLorentz group $\underline{0}(n, 1)[3]$. Because $\underline{O}(n, 1) \cong O(n) \times O(1)$ any element $\Omega$ of $\underline{O}(n, 1)$ is of the form

$$
\Omega=\left(\begin{array}{ll}
P & 0 \\
0 & \varepsilon
\end{array}\right)
$$

where $P \in O(n), \varepsilon= \pm 1$.

A GM point group is defined as a subgroup of $\underline{0}(n, 1)$ which leaves a $n+1-$ dimensional lattice invariant.

The coordinates of a point in the product space with respect to a reference system, which has $n$ orthogonal axes in the $n$-dimensional space and the $(n+1)^{\text {th }}$ axis in the one-dimensional space, are indicated by $x^{1}, x^{2}, \ldots, x^{n+1}$. A GM point group is denoted by $K$, if it is considered as a subgroup of $\underline{o}(n, 1)$. Relative to a basis it is represented as a subgroup of $G L(n+1, R)$. If the basis 
chosen is a basis of a $K$-invariant lattice $\Lambda$, then $K$ may be presented by a subgroup of $G L(n+1, Z)$. In this case we denote it by $\phi(K)$.

Proposition 1:

If the elements $\Omega$ of a $n+1$-dimensional GM point group $\mathrm{K}^{\mathrm{n}+1}$ are of the form (2.1), the elements $P$ of $O(n)$ form a $n$-dimensional Euclidean point group $\mathrm{K}^{\mathrm{n}}$.

Proof: The elements $P$ form a finite subgroup of $O(n)$. So we have only to show that the elements $\mathrm{P}$ leave a n-dimensional lattice invariant. Suppose $K^{n+1}$ leaves invariant a lattice $\Lambda$ with basis vectors $a_{i}=\left[r_{i}, t_{i}\right]$ ( $i=1, \ldots, n+1)$ where $r_{i}=\left(x_{i}^{1}, \ldots, x_{i}^{n}\right), t_{i}=x_{i}^{n+1}$.

For any $t_{0} \neq 0$ the pair of n-dimensional hyperplanes $x^{n+1}= \pm t_{0}$ is transformed onto itself by the elements $\Omega$. The hyperplane $\underline{R}^{n}$, given by $x^{n+1}=0$, is transformed onto itself.

The intersection $\lambda_{k}=\underline{R}^{n} \cap \Lambda$ forms a $k$-dimensional lattice [3, proposition 13] with $0 \leqslant k \leqslant n$. We suppose that $\lambda_{k}$ is generated by $r_{1}, \ldots, r_{k}$. i) If $\mathrm{k}=0$, the only point in $\underline{R}^{\mathrm{n}}$ is the origin. The only point in $\mathrm{x}^{\mathrm{n}+1}=$ $t_{i}$ is $a_{i}$, the only point in $x^{n+1}=-t_{i}$ is $-a_{i}$. If in $(2.1) \varepsilon=1, P$ is an orthogonal transformation which leaves $r_{i}$ fixed for any $i=1, \ldots, n+1$. Hence $P=\mathbb{1}_{n}$. If $\varepsilon=-1, P=-\mathbb{1}_{n}$. Therefore $K^{n}$ is a group generated by $\pm_{n}$, which is clearly a point group.

ii) If $k=n$, the lattice $\lambda_{n}$ is already a n-dimensional lattice left invariant by $P$. So $K^{\mathrm{n}}$ is a $\mathrm{n}$-dimensional point group.

iii) For $0<k<n$, we first consider all elements $\Omega$ with $\varepsilon=+1$. All points in $x^{n+1}=t_{k+i}(i=1, \ldots, n+1-k)$ can be written as $a_{k+i}+[\ell, 0]$ for a certain $\ell \in \lambda_{\mathrm{k}}$. We define $\mathrm{R}^{\mathrm{k}}$, the $\mathrm{k}$-dimensional subspace generated by $\mathrm{r}_{1}, \ldots, \mathrm{r}_{\mathrm{k}}$ and $v_{k+i} \equiv r_{k+i}+R^{k}$, which is a coset of $R^{k}$ in $\underline{R}^{n}$. Then in $x^{n+1}=t_{k+i}$ the orthogonal transformation $P$ leaves invariant the $k$-dimensional lattice $\lambda_{k}$ in $v_{k+i}$. If $R_{\perp}^{n-k} \subset \underline{R}^{n}$ denotes the orthogonal complement of $R^{k}$ in $\underline{R}^{n}$ we define

$$
v_{k+i} \equiv v_{k+i} \cap R_{\perp}^{n-k}
$$

Because $P$ leaves invariant the $k$-dimensional lattice in $\mathrm{v}_{k+i}$ :

$$
P V_{k+i}=V_{k+i}
$$

Because $v_{k+i}$ is the projection of the origin on $v_{k+i}$ it is left invariant by 
the orthogonal transformation $P$.

So the vector $v_{k+i}$ (which is zero if $r_{k+i} \in R^{k}$ ) with components $(0, \ldots, 0$, $\left.v_{k+i}^{k+1}, \ldots, v_{k+i}^{n}\right)$ is left invariant by $P$ for any $i=1, \ldots, n-k+1$. The vectors $r_{k+i}$ and $a_{k+i}$ have components $\left(x_{k+i}^{i}, \ldots, x_{k+i}^{k}, v_{k+i}^{k+1}, \ldots, v_{k+i}^{n}\right)$ and $\left[r_{k+i}, t_{k+i}\right]$ respectively. So if the $n-k+1$ vectors $v_{k+i}$ would generate a real vector space of dimension $d<n-k$, the vectors $a_{i}$ would generate a vector space of dimension $\leqslant k+d+1<n+1$. This not being the case the $n+1-k$ vectors $v_{k+i}$ generate the ( $n-k)$-dimensional subspace $R_{\perp}^{n-k}$. The matrix $P$ leaving invariant each of the vectors $v_{k+i}$ is the identity transformation on $R_{\perp}^{n-k}$. Furthermore it transforms the k-dimensional lattice onto itself, so it has to be of the form

$$
P=\left(\begin{array}{cc}
P_{k} & 0 \\
0 & \mathbb{1}_{n-k}
\end{array}\right)
$$

where the elements $\mathrm{P}_{\mathrm{k}}$ corresponding to all $\mathrm{P}$ form a k-dimensional point group $\mathrm{K}$.

If $\varepsilon=-1$ the same argument is valid in the hyperplane $x^{n+1}=-t_{k+i}$. Therefore all matrices $P$ have the form

$$
P=\left(\begin{array}{cc}
P_{k} & 0 \\
0 & \pm \mathbb{1}_{n-k}
\end{array}\right)
$$

where the matrices $\mathrm{P}_{k}$ form a k-dimensional point group. So the elements $P$ form a n-dimensional point group $\mathrm{K}^{\mathrm{n}}$.

At the same time has been proved:

\section{Proposition 2:}

If a GM point group $\mathrm{K}^{\mathrm{n}+1}$ leaves a lattice $\Lambda$ invariant, the intersection $\Lambda \cap \underline{R}^{n}$ has dimension $\geqslant k$ if and only if the matrices are of the form

$$
\left(\begin{array}{cc}
P_{k} & 0 \\
0 & \pm \mathbb{1}_{n+1-k}
\end{array}\right)
$$

with respect to an orthonormal basis and $\mathrm{P}_{\mathbf{k}}$ form a $\mathrm{k}$-dimensional point group $\mathrm{K}^{\mathrm{k}}$. 
Choose again a basis of the product space consisting of an orthonormal basis in the $n$-dimensional space and the $(n+1)^{\text {th }}$ basis vector in the onedimensional space. We can distinguish three kinds of GM point groups:

i) the first kind, if the group does not contain the time inversion

$$
\left(\begin{array}{cc}
\mathbb{1}_{n} & 0 \\
0 & -1
\end{array}\right)
$$

and all matrices are of the form

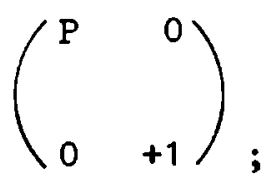

ii) the second kind, if the group does contain the time inversion;

iii) the third kind, if the group $K$ does not contain the time inversion, but there are elements with $\varepsilon=-1(2.1)$. The elements with $\varepsilon=+1$ form a subgroup $\mathrm{K}^{\mathrm{D}}$ of index 2 .

Proposition 3:

For each n+1-dimensional GM point group there exists a n-dimensional Euclidean point group $\mathrm{K}$ such that:

i) If the GM point group is of the first kind, for any $P \in K$ there is an element

$$
\Omega=\left(\begin{array}{cc}
P & 0 \\
0 & +1
\end{array}\right)
$$

and all elements of the GM point group are of this form.

ii) If the GM point group is of the second kind, for any $P \in K$ there are two elements

$$
\Omega_{1}=\left(\begin{array}{cc}
P & 0 \\
& \\
0 & +1
\end{array}\right) \quad \text { and } \quad \Omega_{2}=\left(\begin{array}{cc}
P & 0 \\
& \\
0 & -1
\end{array}\right)
$$


iii) If the $G M$ point group is of the third kind, there exists a subgroup $K^{D} \subset K$ of index two, for any $P^{D} \in K^{D}$ and $P^{D D} \in K-K^{D}$ there are elements

$$
\Omega_{1}=\left(\begin{array}{cc}
P^{D} & 0 \\
& \\
0 & +1
\end{array}\right) \quad \text { and } \quad \Omega_{2}=\left(\begin{array}{cc}
P^{D D} & 0 \\
& \\
0 & -1
\end{array}\right)
$$

respectively and each element of the GM point group is of one of these forms. Proof: $K$ and a fortiori $K K^{D}$ leave invariant a lattice $\lambda_{n}$ with basis vectors $r_{1}, \ldots, r_{n}$. Then all three kinds of groups in $n+1$-dimensions leave invariant the split lattice $\wedge$ generated by $a_{i}=\left[r_{i}, 0\right] \quad(i=1, \ldots, n)$ and $a_{n+1}=$ $\left[0, t_{n+1}\right]\left(t_{n+1}\right.$ arbitrary, real). So they are all GM point groups. (A split lattice is defined in [3]).

Because of propositions 1 and 3 there is a one-to-one correspondence between

i) GM point groups of the first or second kind and n-dimensional trivial magnetic point groups;

ii) GM point groups of the third kind and n-dimensional non-trivial magnetic point groups [11] . So the $n+1$-dimensional abstract GM point groups are isomorphic to these $n$-dimensional point groups.

For that reason for $n+1=4$ there are 25 abstract GM point groups [12] :

$$
\begin{aligned}
& C_{n}(n=1,2,3,4,6) ; C_{n} \times C_{2}(k=4,6), C_{n} \times C_{2} \times C_{2}(k=4,6) \\
& D_{n}(k=2,3,4,6) ; D_{n} \times C_{2}(k=2,4,6), D_{n} \times C_{2} \times C_{2}(k=2,4,6) \\
& T, T \times C_{2}, T \times C_{2} \times C_{2} ; 0,0 \times C_{2}, 0 \times C_{2} \times C_{2} .
\end{aligned}
$$

\section{Proposition 4:}

The GM point groups $K$ and $K^{\prime}$ are GM-geometrically equivalent [3] if and only if the corresponding magnetic groups $K_{m}$ and $K_{m}^{\prime}$ are geometrically equivalent.

Proof: If $K$ and $K^{\prime}$ are geometrically equivalent there is a $\theta \in \underline{O}(n, 1)$ such that $K^{\prime}=\theta K \theta^{-1}$. If $\Omega \in K, \Omega^{\prime} \in K^{\prime}$ such that $\Omega^{\prime}=\theta \Omega \theta^{-1}$, then the matrices $i_{3}^{*} \Omega$ and $i_{3}^{*} \Omega$ (where $i_{3}$ is the injection of the $n+1$-dimensional product space in $\mathrm{n}+1$-dimensional $\mathrm{R}^{\mathrm{n}+1}$, such that the one-dimensional space is mapped on the 
$x^{n+1}$-axis, and $i_{3}^{*}$ is the induced injection $\left.i_{3}^{*}: 0(n, 1) \rightarrow G L(n+1, R)\right)$ are of the form

$$
i_{3}^{*} \Omega=\left(\begin{array}{cc}
P & 0 \\
& \\
0 & E
\end{array}\right) \quad i_{3}^{*} \Omega^{\prime}=\left(\begin{array}{cc}
P^{\prime} & 0 \\
& \\
0 & E^{\prime}
\end{array}\right) \quad\left(\varepsilon, \varepsilon^{\prime}= \pm 1\right)
$$

Because

$$
i_{3}^{*} \Theta=\left(\begin{array}{cc}
Q & 0 \\
0 & \pm 1
\end{array}\right) \quad \text {, where } Q \text { orthogonal, }
$$

one has $P^{\prime}=Q P Q^{-1}$ and $E=E^{\prime}$. This means that $K_{m}$ and $K_{m}^{\prime}$ are geometrically equivalent magnetic point groups.

On the other hand it is likewise simple to see that $K$ and $K^{\prime}$ are geometrically equivalent if $K_{m}$ and $K_{m}^{\prime}$ are geometrically equivalent.

As for $n+1=2,3,4$ the geometric classes of magnetic point groups are known, the GM-geometric classes are also known. They are indicated here by the symbols used in the literature for magnetic crystallographic groups.

\section{Lattices in Euclidean space}

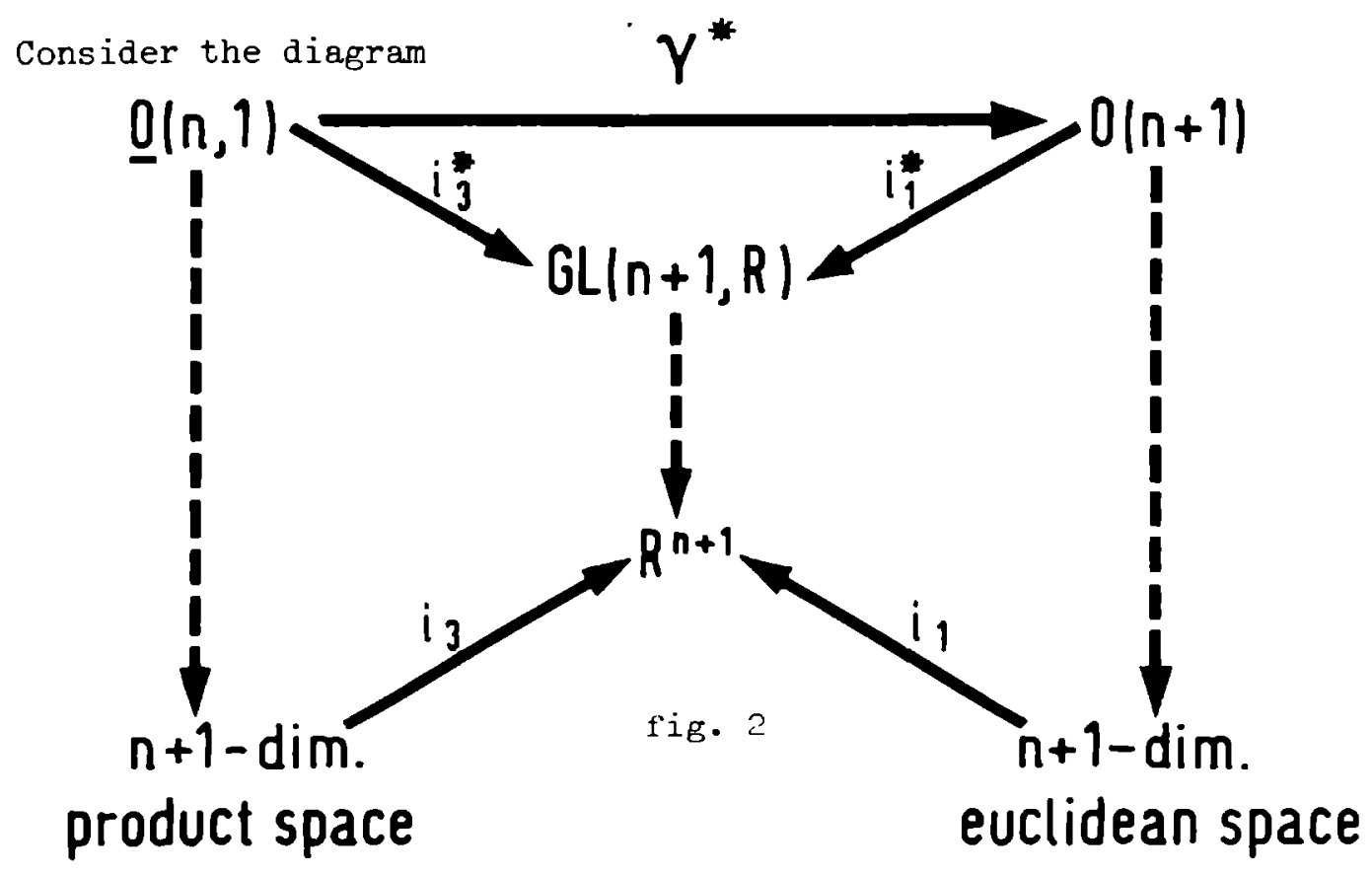


Proposition 5:

If the GM point groups $K$ and $K^{\prime}$ are GM-geometrically equivalent, the groups $\left(i_{1}^{*}\right)^{-1} \circ i_{3}^{*} \circ K$ and $\left(i_{1}^{*}\right)^{-1} \circ i_{3}^{*} \circ K^{\prime}$ are geometrically equivalent Euclidean point groups.

Proof: Because $\left(i_{1}^{*}\right)^{-1} \circ i_{3}^{*} \circ K$ is a subgroup of $0(n+1)$ which leaves a $n+1-$ dimensional lattice invariant it is a Euclidean point group. So is $\left(i_{1}^{*}\right)^{-1} \circ i_{3}^{*} \circ K^{\prime}$. If $K$ and $K^{\prime}$ are equivalent, there is a $T \in \underline{o}(n, 1)$ such that $K^{\prime}=T K T^{-1}$. Define $\gamma^{*}=\left(i_{1}^{*}\right)^{-1} \circ i_{3}^{*}: \underline{o}(n, 1) \rightarrow o(n+1)$. Then $\gamma^{*} \circ K^{\prime}=\left(\gamma^{*} \circ T\right)$ $\left(\gamma^{*} \circ \mathrm{K}\right)\left(\gamma^{*} \circ \mathrm{T}\right)^{-1}$. This means that $\gamma^{*} \mathrm{~K}$ and $\gamma^{*} \mathrm{~K}^{\prime}$ are geometrically equivalent. In general the converse is not true.

Consequence:

It is sufficient to consider all non-equivalent GM-geometrical point groups $K$ and eliminate the Euclidean equivalent ones among the $\gamma^{*} \mathrm{~K}$. In this way the four-dimensional Euclidean geometric crystal classes, corresponding to GM point groups, are found. Of course there are still more four-dimensional geometric crystal classes, which are not considered here.

The arithmetic point groups corresponding to a Euclidean geometric point group are found as $n+1$-dimensional faithful integral representations of these groups. Therefore one has to know the lattices left invariant by these groups. According to [3] it is sufficient to consider one representative of each geometric class with its invariant lattices. Moreover it is not necessary to determine all invariant lattices for each point group but only for their system groups. A system group $K_{0}$ of a point group $K$ is the intersection of all holohedries of lattices left invariant by this point group.

Each lattice is left invariant by the central inversion $-\mathbb{1}_{n+1}$. So the system group $K_{0}$ contains the group generated by $K$ and $-\mathbb{1}_{n+1}$. For $n+1 \leqslant 4$ one has for all point groups, corresponding to a GM point group, the following rules: if $K$ contains a m-fold axis $(m>2)$ a two-dimensional plane in $\underline{R}^{n}$ is an invariant subspace. According to $[13, \mathrm{p} .79]$ a plane lattice with a m-fold symmetry axis, perpendicular to the plane, has also a reflection on a plane through this axis as symmetry element. The group generated by $K,-\mathbb{1}_{n+1}$ and these reflections is a subgroup of the system group.

To find the arithmetic point groups corresponding to a group $K$ one proceeds as follows. If the lattice $\Lambda$ is left invariant by $K$, the intersection $\lambda_{k}=\Lambda \cap \underline{R}^{n}$ is a k-dimensional lattice $(0 \leqslant k \leqslant n)$ as seen in section 2 . If one chooses $k$ basis vectors of $\lambda_{k}$ as first $k$ basis vectors of $\Lambda$ and if the 
basis vectors of $\Lambda$ are $a_{i}=\left[r_{1}, t_{i}\right](i=1, \ldots, n+1)$, the lattice $\Lambda$ is $a$ repetition of $\lambda_{k}$ in each hyperplane

$$
x^{n+1}=\sum_{i=k+1}^{n+1} m_{i} t_{i} \quad\left(m_{i} \in z\right) .
$$

According to proposition 2, if the matrices of $K$ are of the form (2.3), dim $\lambda_{k} \geqslant k$. We suppose now $K_{o}$ to be a system group. We consider $K_{o}^{+}$, which is the subgroup of index two which does not contain $-\mathbb{1}_{n+1}$. According to the proof of proposition 1 (section 2) $\mathrm{K}_{0}^{+}$leaves the point $\left[\mathrm{v}_{\mathrm{k}+i}, \mathrm{t}_{\mathrm{k}+\mathrm{i}}\right]$ fixed $(i=1, \ldots, n+1-k)$ and the point set $\left[r_{k+i}+\lambda_{k}, t_{k+i}\right]$ invariant. There is an unique $\bar{r}_{k+i} \in v_{k+i}$ (notation of section 2) such that $r_{k+i}=\bar{r}_{k+i}+v_{k+i}$. The vector $\bar{r}_{k+i}$ has $n$ components: $\left(\bar{x}_{k+i}^{1}, \ldots, \bar{x}_{k+i}^{k}, 0, \ldots, 0\right)$ and $v_{k+i}$ has $n$ components $\left(0, \ldots, 0, v_{k+i}^{k+1}, \ldots, v_{k+i}^{n}\right)$. So $K^{k}$ consisting of all matrices $P_{k}$ in the elements $\Omega$ of $\mathrm{K}_{\mathrm{o}}^{+}$leaves invariant $\bar{\lambda}=\overline{\mathrm{r}}_{k+i}+\lambda_{k}$ in $R_{n}$ and $\mathrm{K}^{\mathrm{k}} \overline{\mathrm{r}}_{\mathrm{k}+\mathrm{i}}=\overline{\mathrm{r}}_{\mathrm{k}+i}$ $\left(\bmod \lambda_{k}\right)$. This means that $\bar{r}_{k+i}$ can be chosen as origin of the lattice, or that $\bar{r}_{k+i}$ is a point with the same point symmetry as the origin. For each $\lambda_{k}$ left invariant by $\mathrm{K}^{\mathrm{k}}$ the vectors in the unit cell with the same symmetry as the origin are listed for $k \leqslant 3$ in [14]. The positions are given by coordinates, relative to the lattice basis vectors, called lattice coordinates. For a point in the unit cell $0 \leqslant \alpha<1$ for each lattice coordinate.

One can distinguish two cases:

i) The points in the unit cell with the same point symmetry as the origin form a discrete set.

ii) These points form a continuous (not necessarily connected) set.

To find the invariant lattices one proceeds as follows:

Case i)

If $\bar{r}_{k+i}=0(i=1, \ldots, n+1-k)$, then $r_{k+i}=v_{k+i}$ and $a_{k+i}=\left[v_{k+i}, t_{k+i}\right] \stackrel{\text { def }}{\equiv}$ $\bar{a}_{k+i}$. If $\bar{r}_{k+i}=\left(\sigma_{k+i}^{l}, \ldots, \sigma_{k+i}^{k}\right) \neq 0$ in lattice coordinates, consider the smallest positive integer $\mathrm{m}_{k+i}$ such that $\mathrm{m}_{k+i} \overline{\mathbf{r}}_{\mathrm{k}+i} \in \lambda_{\mathrm{k}}$. Then

$$
\begin{aligned}
m_{k+i}\left[v_{k+i}, t_{k+i}\right] & =m_{k+i}\left[r_{k+i}-\bar{r}_{k+i}, t_{k+i}\right]= \\
& =m_{k+i} a_{k+i}-m_{k+i}\left[\bar{r}_{k+i}, 0\right] \in \Lambda .
\end{aligned}
$$

Choose $m_{k+i}\left[v_{k+i}, t_{k+i}\right]$ as basis vector $\bar{a}_{k+i}$ of a sublattice $\Lambda_{0} \subseteq \Lambda$. The lattice $\Lambda_{0}$ is characterized by its metric tensor $g$ with components $g_{i j}=\bar{a}_{i}, \bar{a}_{j}$ 
$(i, j=1, \ldots, n+1)$. If $g(k)$ is the metric tensor of $\lambda_{k}$

$$
\bar{a}_{i} \cdot \bar{a}_{j} \begin{cases}=g(k)_{i j} & (i, j=1, \ldots, k) \\ =r_{i} \cdot v_{j}=0 & (i \leqslant k, j>k) \\ =\text { arbitrary real number }(i>k, j>k)\end{cases}
$$

Hence

$$
g=\left|\begin{array}{ccc}
g(k) & 0 & \\
& a_{k+1} \cdot a_{k+1} & \vdots \\
0 & \vdots & \vdots \\
& a_{n+1} \cdot a_{k+1} \ldots-a_{k+1} \cdot a_{n+1}
\end{array}\right|
$$

Because dim $\lambda_{k}=k$ one has

$$
t_{i}=0 \quad(i \leqslant k)
$$

$$
\sum_{j=k+1}^{n+1} \quad c_{j} t_{j} \neq 0 \quad\left(\text { any } c_{j} \in Z\right)
$$

However the basis transformation $a_{i} \longmapsto \bar{a}_{i}$ is not unimodular if $\bar{r}_{k+i} \neq 0$ for some $i$. Hence the lattice spanned by $\bar{a}_{i}(i=1, \ldots, n+1)$ and determined by (3.1) is in general a non-primitive lattice. The interior points are generated by $s_{k+i}=\left[v_{k+i}+\bar{r}_{k+i}, t_{k+i}\right]$ for those values of $i$ for which $\bar{r}_{k+i} \neq 0$. Decause $\left[v_{k+i}, t_{k+i}\right]=\frac{1}{m_{k+i}} \bar{a}_{k+i}$ in this case, the lattice coordinates of $\mathrm{s}_{\mathrm{k}+\mathrm{i}}$ are:

$$
s_{k+i}=\left(\sigma_{k+i}^{1}, \ldots, \sigma_{k+i}^{k}, 0, \ldots, 0, \frac{1}{{\underset{k}{k+i}}_{(k+i)} \text { th }}\right. \text { lattice coordinate }
$$

\section{Cese ii)}

$\bar{r}_{k+i}=\left[\tau_{k+i}^{l}, \ldots, \tau_{k+i}^{r}, \sigma_{k+i}^{r+1}, \ldots, \sigma_{k+i}^{n}\right]$

where $0 \leqslant \sigma_{k+i}^{j}<1$ such that $m_{k+i}\left(0, \ldots, 0, \sigma_{k+i}^{r+1}, \ldots, \sigma_{k+i}^{n}\right)=0\left(\bmod \lambda_{k}\right)$ and 
$0 \leqslant \tau_{k+i}^{j}<1$ real parameters.

a) Suppose $\sigma_{k+i}^{j}=0 \quad(j=r+1, \ldots, k)$

Then $\left[\tau_{k+i}^{1} r_{1}+\ldots+\tau_{k+i}^{r} r_{r}+v_{k+i}, t_{k+i}\right] \in \Lambda$. Take this as a basis vector $a_{k+i}$. Then the metric tensor $g$ is given by

$$
B_{i j}=\left\{\begin{array}{cc}
g(k)_{i j} & (i, j \leqslant k) \\
a_{i} \cdot a_{j} & (i, j>k) \\
\sum_{\ell=1}^{r} \tau_{j}^{\ell} a_{i} \cdot a_{\ell}= & \sum_{\ell=1}^{r} \tau_{j}^{\ell} r_{i} \cdot r_{\ell}
\end{array}\right.
$$

So

$$
g=\left|\begin{array}{ccc}
g(k) & f & \\
& a_{k+1} \cdot a_{k+1} \ldots \ldots a_{k+1} \cdot a_{n+1} \\
f^{t} & \vdots & \vdots \\
& a_{k+1} \cdot a_{n+1} \ldots \ldots & \vdots \\
& & \vdots \ldots
\end{array}\right|
$$

where $f$ is a $k \times(n+1-k)$ matrix with elements (3.3). $g_{i j}=0(i \leqslant k, k+1 \leqslant j \leqslant n+1)$ only if $r_{i} \cdot r_{\ell}=0$ for all $\ell \leqslant r$. b) If not all $\sigma_{k+i}^{j}=0$ the points in the unit cell with the same symmetry as the origin of $\lambda_{k}$ form a disconnected continuous set. Again take

$$
\bar{a}_{k+i}=m_{k+i}\left[\tau_{k+i}^{l} x_{1}+\ldots+\tau_{k+i}^{r} x_{r}+v_{k+i}, t_{k+i}\right]
$$

as a basis vector of the sublattice $\Lambda_{0} \subseteq \Lambda$.

Then the metric tensor of the (non-primitive) lattice is given by (3.3). It has a set of interior points generated by vectors with lattice coordinates $\left(0, \ldots, 0, \sigma_{k+i}^{r+1}, \ldots, \sigma_{k+i}^{k}, 0, \ldots, \frac{1}{m_{k+i}}, 0, \ldots, 0\right)=s_{k+i}$.

In this way one has proved the following proposition which can be applied to find all lattices left invariant by a given point group.

Proposition 6:

If a point group $K$ leaves invariant a lattice $\Lambda$ such that for the $k-$ dimensional lattice $\lambda_{k}=\Lambda \cap \underline{R}^{n}$ the points in the unit cell of $\lambda_{k}$ with the 
same symmetry as the origin are $\{\bar{r}\}$ one has the following four possibilities for $\Lambda_{0} \subseteq \Lambda$.

i) If the points $\{\bar{r}\}$ form a discrete set, the metric tensor $g$ of $\Lambda_{0}$ has the form

$$
g=\left(\begin{array}{cc}
g(k) & 0 \\
0 & g_{(n+1-k)}
\end{array}\right)
$$

where $g(k)$ is the metric tensor of $\lambda_{k} ; g_{(n+1-k)}$ is arbitrary symmetric;

a) $\Lambda_{0}$ is a primitive lattice $\left(\Lambda_{0}=\Lambda\right)$; or

b) it is possible to choose $\bar{r}_{k+i}=\left(\sigma_{k+i}^{l}, \ldots, \sigma_{k+i}^{k}\right) \neq 0$ for some $i \leqslant n+1-k$ and the interior points of $\Lambda_{0}$ are generated by points with lattice coordinates

$$
\alpha_{k+i}^{j}= \begin{cases}\sigma_{k+i}^{j} & (j \leqslant k) \\ 1 / m_{k+i} & \left(j=i+k \text { if } \bar{r}_{k+i} \neq 0\right) \\ 0 & \text { (otherwise) }\end{cases}
$$

ii) If the points $\{\bar{r}\}$ form a continuous set: $\bar{r}=\left[\tau^{1}, \ldots, \tau^{r}, \sigma^{r+1}, \ldots, \sigma^{k}\right]$ $\left(\tau^{j} \in R, \sigma^{\ell} \in Q\right)$ the metric tensor $g$ of $\Lambda_{0}$ has the form

$$
g=\left(\begin{array}{cc}
g(k) & f \\
f^{t} & g(n+1-k)
\end{array}\right)
$$

where $g(k)$ is the metric tensor of the $\lambda_{k} ; g(n+1-k)$ is arbitrary symmetric; and $g_{i j}=0(i \leqslant k, k+1 \leqslant j \leqslant n+1)$ only if $r_{i} \cdot r_{\ell}=0$ for all $\ell \leqslant r$.

a) $\Lambda_{0}$ is a primitive lattice $\left(\Lambda_{0}=\Lambda\right)$; or

b) it is possible to choose $\bar{r}_{k+i}=\left(0, \ldots, 0, \sigma_{k+i}^{r+1}, \ldots, \sigma_{k+i}^{k}\right) \neq 0$ for some $i \leqslant n+1-k$ and its interior points are generated by points with lattice coordinates 
$\alpha_{k+i}^{j}=\left\{\begin{array}{cl}\sigma_{k+i}^{j} & (r+1 \leqslant j \leqslant k) \\ 1 / m_{k+i} & \left(j=k+i \text { if } \bar{r}_{k+i} \neq 0\right) \\ 0 & \text { (otherwise) }\end{array}\right.$

Consider as an example of the first case the 3-dimensional Euclidean point group $4 / \mathrm{mmm}$ which corresponds to a GM point group. If $\Lambda$ is a lattice left invariant, $\operatorname{dim}\left(\Lambda \cap \underline{R}^{2}\right)=2$ and $K^{2}$ is the plane point group $4 \mathrm{~mm}$ which leaves invariant a plane tetragonal lattice. The points in the unit cell of this lattice with the same point symmetry as the origin are $(00)$ and $\left(\frac{1}{2} \frac{1}{2}\right)$. The metric tensor of the lattice $\Lambda_{0}$ is

$$
\left(\begin{array}{ccc}
a_{1}^{2} & 0 & 0 \\
0 & a_{1}^{2} & 0 \\
0 & 0 & a_{3}^{2}
\end{array}\right)
$$

If $r_{3}=\bar{r}_{3}=(00)$, one has a primitive lattice $\left(\Lambda=\Lambda_{0}\right)$ and if $r_{3}=\left(\frac{1}{2} \frac{1}{2}\right)$ one has a centered lattice. Hence a lattice left invariant by $4 / \mathrm{mmm}$ is a primitive or a body centered tetragonal lattice ( $f i g .3 a$ and $3 b$ )

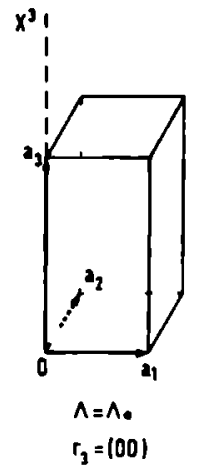

(a)

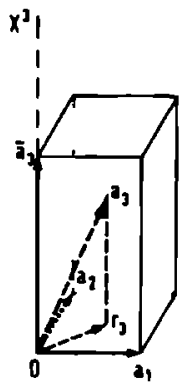

A.cA $r_{3}=\left(\frac{1}{2} \frac{1}{2}\right)$

(b)

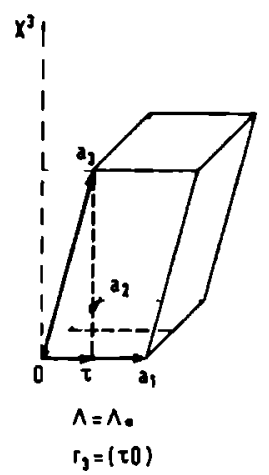

(c)

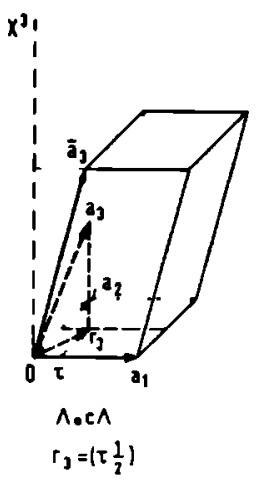

(d)

fig. 3 
As an example of the second case consider the Euclidean 3-dimensional point group $m$. Here $\operatorname{dim}\left(\Lambda \cap \underline{R}^{2}\right)=1$ or 2 . Take the case $k=2$, then $K^{2}$ is the point group m which leaves invariant a rectangular or a rhombohedral lattice. If $\lambda_{2}$ is rectangular the points with the same symmetry as the origin are $\left(\begin{array}{ll}\tau & 0\end{array}\right)$ and $\left(\tau \frac{1}{2}\right)$, where $\tau$ is an arbitrary real number $(0 \leqslant \tau<1)$. As $\bar{a}_{1} \cdot \bar{a}_{3}=$ $r_{1} \cdot \tau r_{1}=\tau r_{1}^{2}$ and $\bar{a}_{2} \cdot \bar{a}_{3}=\tau r_{1} \cdot r_{2}=0$ the metric tensor of $\Lambda_{0}$ is

$$
\left(\begin{array}{ccc}
a_{1}^{2} & 0 & a_{1} \cdot a_{3} \\
0 & a_{2}^{2} & 0 \\
a_{1} \cdot a_{3} & 0 & a_{3}^{2}
\end{array} \mid\right.
$$

If $r_{3}=\left(\begin{array}{ll}\tau & 0\end{array}\right), \Lambda=\Lambda_{0}$, and if $r_{3}=\left(\tau \frac{1}{2}\right), \Lambda_{0}$ is centered with interior point $\left(0 \frac{1}{2}\right)$. So one finds a primitive and a side-centered monoclinic lattice. (figs. $3 c$ and $3 d$ ).

\section{Four-dimensional Bravais classes and arithmetic crystal classes correspon-} ding to GM point groups

By means of the propositions 1 to 6 of section 2 and 3 we are now able to find the four-dimensional lattices with a holohedry corresponding to a GM point group and the arithmetic crystal classes corresponding to such groups. Because of the one-to-one correspondence between GM point groups and magnetic groups of one dimension less, trivial ones included, there are 122 geometrically non-equivalent four-dimensional GM point groups which give rise, by way of the mapping $\left(i_{1}^{*}\right)^{-1} i_{3}^{*}$ of section 3 , to the same number of Euclidean geometric point groups. Among these are 88 geometrically non-equivalent ones, as one verifies easily using the fact that in Euclidean space reflections along the fourth axis do not play another role than reflections along the other axes. The 88 classes, found in this way, are the crystal classes which are $(3+1)$-reducible over $R$.

According to Hurley $[7,8]$ there are 182 reducible and 45 irreducible classes in the 4-dimensional Euclidean case. Hence the number of 2+2-reducible crystal classes is 94 . 
The 88 classes corresponding to GM point groups are given in table $I$.

From each geometric class we choose a representative group $K$ and construct from this a group $\bar{K}$ generated by $K$, the central inversion and a reflection on a plane through 3,4 and 6-fold axes. If a lattice is left invariant by $K$ it is also left invariant by a group geometrically equivalent to $\bar{K}$. For this group $\overline{\mathrm{K}}$ we construct all invariant lattices using the method of section 3. The maximal point group which leaves invariant all lattices left invariant by $K$ is by definition the system group $K_{0}$. The system group for each geometric crystal class is given in table $I$. In the present case they are exactly the geometric classes of the holohedries.

Among all lattices left invariant by a system group $K_{0}$ we consider those for which $K_{0}$ is the geometric holohedry. All lattices which after a conveniently chosen basis transformation have a geometric tensor of the same form, in other words for which the arithmetic representations $\phi\left(K_{0}\right)$ are arithmetically equivalent, form together a Bravais class. So from all system groups corresponding to GM point groups we obtain the Bravais classes with a holohedry corresponding to a GM point group. These are given in table II. If two arithmetic holohedries $\phi(H)$ and $\bar{\phi}(H)$ are geometrically equivalent they are also conjugate in $G L(n, Q)$ because $\phi(H)$ and $\bar{\phi}(H)$ are groups of integral matrices. Hence Bravais classes with the same geometric holohedry (so they belong to the same system) are centerings of each other. Moreover some lattices may be presented as centering of a lattice with larger holohedry. The 41 Bravais classes with a holohedry corresponding to a four-dimensional GM point group are given in table II. The other four-dimensional Bravais classes have a holohedry which does not correspond to a GM point group.

In general one may define an orientation for lattices in even dimensions: two lattices in the same Bravais class have the same orientation if their arithmetic holohedries are conjugated by an element of $\operatorname{SL}(n, Z)$.

Lemma:

All lattices of a Bravais class have the same orientation if and only if the normalizer $\mathrm{N}_{\phi(H)}$ of one representative holohedry $\phi(H)$ in $G L(n, Z)$ contains an element $\xi$ with $\operatorname{det}(\xi)=-1$.

Proof: Consider two holohedries $\phi_{1}(H)$ and $\phi_{2}(H)$ of lattices in the Bravais class which are conjugated by $x \in G L(n, z)$ with $\operatorname{det}(x)=-1$.

Suppose there is an element $\xi \in \mathbb{N}_{\phi_{1}}(H)$ with $\operatorname{det}(\xi)=-1$. As $\phi_{1}(H)=\chi \phi_{2}(H) x^{-1}$, one has 


$$
\phi_{1}(H)=\xi \phi_{1}(H) \xi^{-1}=\xi \times \phi_{2}(H)(\xi \times)^{-1}
$$

Because det $(\xi x)=+1$ the corresponding lattices have the same orientation. Conversely, if all lattices of the Bravais class have the same orientation for any two holohedries $\phi_{1}(H)$ and $\phi_{2}(H)$ in the class one has an element with

$$
\phi_{1}(H)=n \phi_{2}(H) n^{-1} \text { and } \operatorname{det}(n)=+1 \text {. }
$$

Take $\phi_{2}(H)=x \phi_{1}(H) x^{-1}$ with $x \in G L(n, Z)$ and $\operatorname{det}(x)=-1$. Then $\phi_{1}(H)=n x \phi_{1}(H)(n x)^{-1}$. So $n x \in \mathbb{N}_{\phi_{1}(H)}$ and $\operatorname{det}(n x)=-1$.

All lattices in table II except those in the classes I, III P, III I, III $G$ and IX have a holohedry $\phi(H) \subseteq \mathbb{N}_{\phi}(H)$ with an element $\xi$ with $\operatorname{det}(\xi)=-1$. The five remaining classes have elements of the normalizers of the holohedry with this property. So all lattices in table II are non-orientable.

If one knows for each geometric group $K$ its system group $K_{0}$ and the Bravais classes left invariant by $K_{0}$, it is possible to determine the arithmetic classes corresponding to each geometric class. The first step is to determine the arithmetic crystal classes R-equivalent to a given system group $K_{0^{-}}$Each Bravais class left invariant by $K_{0}$ and for which $K_{0}$ is the holohedry gives one arithmetic crystal class R-equivalent to $K_{0}$. A Bravais class left invariant by $\mathrm{K}_{0}$ with a holohedry $\phi(\mathrm{H})$ which does not contain a subgroup arithmetically equivalent to the holohedry of another Bravais class left invariant by $K_{0}$, gives one or more non-equivalent integral representations of $K_{0}$. The number of non-equivalent arithmetic crystal classes is the number of arithmetically non-equivalent subgroups of $\phi(H)$ which are R-equivalent to $K_{0}$. If the holohedry $\phi(H)$ of a Bravais class left invariant by $K_{0}$ does contain a subgroup arithmetically equivalent to the holohedry of another Bravais class it does not give rise to other non-equivalent arithmetic crystal classes. As all arithmetic point groups $R$-equivalent to $K_{0}$ are also $Q$-equivalent, where $Q$ is the rational number field, the arithmetic crystal classes R-equivalent to a group $K$ may be obtained as representations of $K$ on bases of lattices which are centerings of each other.

The arithmetic crystal classes corresponding to a point group $K$ are found as the arithmetically non-equivalent subgroups (R-equivalent to $K$ ) of the arithmetic point groups R-equivalent to the system group $\mathrm{K}_{\mathrm{O}}$. In this way 
one finds 412 arithmetic crystal classes corresponding to the 88 geometric classes listed in table $I$. Owing to the remark made at the end of the last paragraph the arithmetic point groups corresponding to a geometric point group $\mathrm{K}$ are Q-equivalent.

So for each geometric class the arithmetic crystal classes are given by generators for one group and transformation matrices from $G L(n, Q)$ for the other ones. If $\phi(\alpha)$ is an element from one arithmetic point group, and if the transformation matrix is $T$ the corresponding element in a second arithmetic point group is given by

$$
\bar{\phi}(\alpha)=T \phi(\alpha) T^{-1} \quad T \in G L(n, Q)
$$

For each of the 88 geometric crystal classes generators for one arithmetic point group and matrices $T \in G L(4, Q)$ are given in table $I$.

So limiting oneself to (3+1)-reducible point groups one finds in four dimensions 412 arithmetic crystal classes grouped in 88 geometric crystal classes belonging to 25 isomorphism classes and 41 Bravais classes classified in 15 systems. 


\section{Table I}

Georetric and arithmétic crystal classes corresponding to 4-dimensional GM point eroups

\begin{tabular}{|c|c|c|c|c|c|c|c|}
\hline$\left[\begin{array}{l}\text { System } \\
\text { tahle }\end{array}\right]$ & $\begin{array}{l}\text { Georetric } \\
\text { class }\end{array}$ & $\begin{array}{l}\text { Isomorphism } \\
\text { class } \\
\text { [tabie III ] }\end{array}$ & Order & $\begin{array}{l}\text { Generators } \\
{[\text { table IV }]}\end{array}$ & 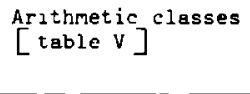 & $\begin{array}{l}\text { Number } \\
\text { arththm. } \\
\text { cl. }\end{array}$ & $\begin{array}{l}\text { Numbering } \\
\text { arithm. cl." }\end{array}$ \\
\hline$I \bar{l}$ & $\begin{array}{l}1 \\
\overline{1}\end{array}$ & & $\begin{array}{l}1 \\
2\end{array}$ & $\begin{array}{l}1 \\
2\end{array}$ & $\begin{array}{l}\mathrm{P} \\
\mathrm{P}\end{array}$ & $\begin{array}{l}1 \\
1\end{array}$ & $\begin{array}{l}1 \\
2\end{array}$ \\
\hline II $T 1$, & $\begin{array}{l}\bar{T}=2^{\prime} \\
1^{\prime}=\mathrm{m} \\
\overline{1}^{\prime}{ }^{\prime}=21 / \mathrm{m}\end{array}$ & $\begin{array}{l}\mathrm{C}_{2} \\
\mathrm{C}_{2} \\
\mathrm{D}_{2}\end{array}$ & $\begin{array}{l}2 \\
2 \\
4\end{array}$ & $\begin{array}{l}31 \\
25 \\
3125\end{array}$ & $\begin{array}{ll}\text { P I } \\
\text { F I } \\
\text { P I }\end{array}$ & $\begin{array}{l}2 \\
2 \\
2\end{array}$ & $\begin{array}{l}3 \\
5 \\
7\end{array}$ \\
\hline $\operatorname{III} 2 / \pi^{\prime}$ & $\begin{array}{l}2=\pi^{\prime} \\
2 / m^{\prime}\end{array}$ & $\begin{array}{l}C_{2} \\
D_{2}\end{array}$ & $\begin{array}{l}2 \\
4\end{array}$ & $\begin{array}{ll}3 & \\
3 & 4\end{array}$ & $\begin{array}{lll}\text { P } & \text { I } & G \\
\text { F } & \text { I } & G\end{array}$ & $\begin{array}{l}3 \\
3\end{array}$ & $\begin{array}{r}9 \\
12\end{array}$ \\
\hline IV $2 /\left.\mathrm{m}\right|^{\prime}$ & 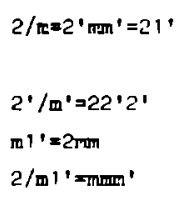 & $\begin{array}{l}\mathrm{D}_{2} \\
\mathrm{D}_{2} \\
\mathrm{D}_{2} \\
\mathrm{D}_{2} \\
\mathrm{D}_{2} \times \mathrm{C}_{2}\end{array}$ & $\begin{array}{l}4 \\
4 \\
4 \\
8\end{array}$ & $\begin{array}{rrr}4 & 27 \\
4 & 25 \\
3 & 38 \\
25 & 27 & \\
25 & 27 & 4\end{array}$ & 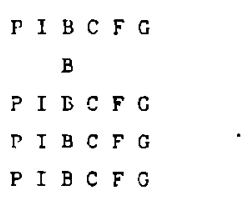 & $\begin{array}{l}7 \\
6 \\
6 \\
6\end{array}$ & $\begin{array}{l}15 \\
21 \\
22 \\
28 \\
34\end{array}$ \\
\hline VI $\overline{3} \cdot m$ & $\begin{array}{l}3 \\
3 \\
322^{\prime} \\
3 \mathrm{~m} \\
\overline{3}^{\prime} \mathrm{m}\end{array}$ & $\begin{array}{l}C_{1} \\
C_{6} \\
D_{3} \\
D_{1} \\
D_{3} \\
D_{3} \\
D_{6} \\
D_{6}\end{array}$ & $\begin{array}{l}3 \\
6 \\
6 \\
6 \\
12\end{array}$ & $\begin{array}{l}15 \\
37 \\
1533 \\
1532 \\
1528 \\
1529 \\
3728 \\
3729\end{array}$ & $\begin{array}{l}P R \\
P R \\
P R \\
P \\
P \\
P R \\
P \\
P R\end{array}$ & $\begin{array}{l}2 \\
2 \\
3 \\
3 \\
3\end{array}$ & $\begin{array}{l}40 \\
42 \\
44 \\
46 \\
47 \\
48 \\
50 \\
51\end{array}$ \\
\hline v $6 / m^{\prime} \mathrm{mm}$ & $\begin{array}{l}6 \\
6, \\
6 / \mathrm{m}^{\prime} \\
6 \mathrm{~mm} \\
6 \cdot \mathrm{m}^{\prime} \cdot \\
62 \cdot 2^{\prime} \\
6 / \mathrm{m}^{\prime} \mathrm{mm}\end{array}$ & $\begin{array}{l}C_{6} \\
C_{6} \\
C_{6} \times C_{2} \\
D_{6} \\
D_{6} \\
D_{6} \\
D_{6} \\
D_{6}=C_{2}\end{array}$ & $\begin{array}{r}6 \\
6 \\
12 \\
12 \\
12 \\
12 \\
24\end{array}$ & 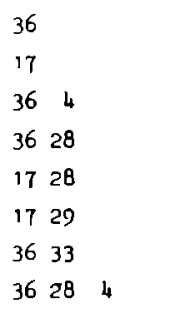 & $\begin{array}{l}P \\
P \\
P \\
P \\
P \\
P \\
P \\
P\end{array}$ & $\begin{array}{l}1 \\
1 \\
1 \\
1 \\
2 \\
1 \\
1\end{array}$ & $\begin{array}{l}53 \\
54 \\
55 \\
56 \\
57 \\
58 \\
59 \\
60\end{array}$ \\
\hline VII $4 / \pi^{\prime} m m$ & $\begin{array}{l}4 \\
4 \cdot \\
4 / m^{\prime} \\
4 m m \\
4 \cdot 2 \cdot m \\
42 \cdot 2 \cdot \\
4 / m^{\prime} \mathrm{mm}\end{array}$ & $\begin{array}{l}C_{4} \\
C_{4} \\
C_{4} \times C_{2} \\
D_{4} \\
D_{4} \\
D_{4} \\
D_{4} \\
D_{4} \times C_{2}\end{array}$ & $\begin{array}{c}4 \\
4 \\
8 \\
8 \\
8 \\
\\
8 \\
16\end{array}$ & 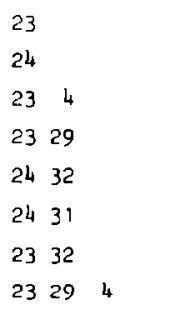 & $\begin{array}{ll}\text { F } & I \\
E & I \\
P & I \\
P & \text { I } \\
P & I \\
P & I \\
P & I \\
P & I\end{array}$ & $\begin{array}{l}\hat{2} \\
2 \\
2 \\
2 \\
4 \\
2 \\
2\end{array}$ & $\begin{array}{l}61 \\
63 \\
65 \\
67 \\
69 \\
71 \\
73 \\
75\end{array}$ \\
\hline
\end{tabular}

-) Number of arithmetic classes geometrically equivalent to the given geometric class. *) Given is the number of the first arithmetic class in the row. E.g. arithmetic class 43 is $R^{\top} \mathrm{H}$, which is generated by $T a T^{-1}$, vhere $a=$ gatrix 37 (table IV) and $T=$ matrix $R$ (table $V$ ). 


\begin{tabular}{|c|c|c|c|c|c|c|c|}
\hline $\begin{array}{l}\text { System } \\
{[\text { toble II }]}\end{array}$ & $\begin{array}{r}\text { Geometric } \\
\text { class }\end{array}$ & $\begin{array}{l}\text { Isomorphism } \\
\text { class } \\
{[\text { table III }]}\end{array}$ & Order & $\begin{array}{l}\text { Generators } \\
{[\text { table IV }]}\end{array}$ & $\begin{array}{l}\text { Arithmetic classes } \\
{[\text { table v }]}\end{array}$ & $\begin{array}{l}\text { Number } \\
\text { arithr. } \\
\text { cl. }\end{array}$ & $\begin{array}{l}\text { Numbering } \\
\text { arithm. cl. }\end{array}$ \\
\hline$I X m^{\prime} m^{\prime} m^{\prime}$ & $\begin{array}{l}222=n^{\prime} m^{\prime} 2 \\
m^{\prime} m^{\prime} m^{\prime}\end{array}$ & $\begin{array}{l}\mathrm{D}_{2} \\
\mathrm{D}_{2} \\
\mathrm{D}_{2} \\
\mathrm{D}_{2} \times \mathrm{C}_{2}\end{array}$ & 4 & $\begin{array}{lll}3 & 5 & \\
5 & 6 & \\
6 & 7 & \\
6 & 7 & 4\end{array}$ & $\begin{array}{l}\text { P I A E F G H S K } \\
\text { A } \\
\text { E I A E F } \\
\text { P H } S K\end{array}$ & 13 & $\begin{array}{l}77 \\
86 \\
88 \\
90\end{array}$ \\
\hline$V I \perp I m m 1^{\prime}$ & $\begin{array}{l}m m m=m m 21^{\prime} \\
m^{\prime} m^{\prime} r=2221^{\prime} \\
m m m^{\prime \prime}\end{array}$ & $\begin{array}{l}\mathrm{J}_{2} \times \mathrm{C}_{2} \\
\mathrm{~J}_{2} \times \mathrm{C}_{2} \\
\mathrm{D}_{2} \times \mathrm{C}_{3} \\
\mathrm{D}_{2} \times \mathrm{C}_{2} \\
\mathrm{D}_{2} \propto \mathrm{C}_{2} \\
\mathrm{I}_{2} \times \mathrm{C}_{2} \\
\mathrm{~L}_{2} \times \mathrm{C}_{2} \times \mathrm{C}_{2}\end{array}$ & 3 & $\begin{array}{rrrr}25 & 26 & 27 \\
25 & 26 & 30 \\
25 & 27 & 30 \\
6 & 7 & 26 \\
6 & 4 & 27 \\
3 & 12 & 30 \\
25 & 26 & 27 & 30\end{array}$ & $\begin{array}{l}\text { PIA E F O H S } \\
\text { A E F } \\
\text { PI A E F C F S } \\
\text { A } \\
\text { F F } \\
\text { PI A E F G H S }\end{array}$ & 12 & $\begin{array}{r}99 \\
107 \\
109 \\
111 \\
119 \\
121 \\
123\end{array}$ \\
\hline$X I \overline{3} m 1$ ' & $\begin{array}{l}\overline{3}=6{ }^{\prime} \\
31^{\prime}=6 \\
\overline{3} 1^{\prime}=61^{\prime} / \mathrm{m} \\
32=3 \mathrm{~m}^{\prime} \\
\overline{3} \mathrm{~m}=6{ }^{\prime} \mathrm{mm}{ }^{\prime} \\
\overline{3} \mathrm{~m}^{\prime}=6{ }^{\prime} 22^{\prime} \\
\overline{3}{ }^{\prime} \mathrm{m}^{\prime} \\
321^{\prime}=6 \mathrm{~m}^{\prime} 2^{\prime} \\
3 \mathrm{~m} 1^{\prime}=6 \mathrm{m2} \\
\overline{3} \mathrm{~m} 1^{\prime}=6{ }^{\prime} / \mathrm{mm} \cdot\end{array}$ & $\begin{array}{l}C_{6} \\
C_{1} \times C_{2} \stackrel{2}{=} C_{6} \\
C_{6} \times C_{2} \\
D_{2} \\
D_{3} \\
D_{3} \\
D_{6} \\
D_{6} \\
D_{6} \\
D_{6} \\
D_{6} \\
D_{3} \times C_{2} \stackrel{2}{=} D_{6} \\
D_{1} \times C_{3} \\
D_{1} \times C_{2}=D_{6} \\
D_{3} \times C_{2} \\
D_{0} \times C_{2} \\
D_{6} \times C_{2}\end{array}$ & $\begin{array}{l}12 \\
12 \\
12 \\
\cdot 2 \\
12 \\
24\end{array}$ & 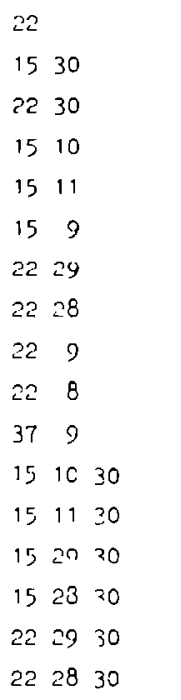 & 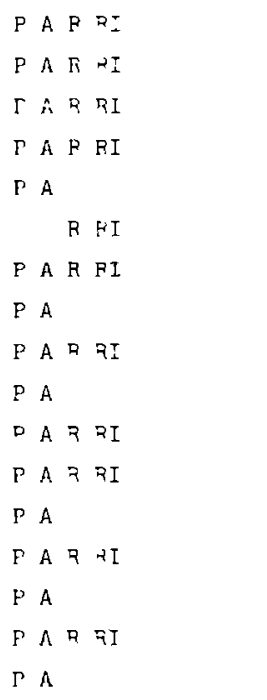 & $\begin{array}{l}4 \\
6 \\
6 \\
6\end{array}$ & $\begin{array}{l}131 \\
135 \\
139 \\
143 \\
147 \\
149 \\
151 \\
155 \\
157 \\
161 \\
163 \\
167 \\
171 \\
173 \\
177 \\
179 \\
183\end{array}$ \\
\hline$x 6 / m m m 1$ ' & 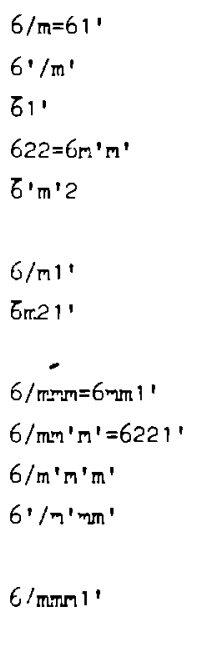 & $\begin{array}{l}C_{6} \times C_{2} \\
C_{6} \times C_{2} \\
C_{6} \times C_{2} \\
D_{6} \\
D_{6} \\
D_{6} \\
C_{6} \times C_{2} \times C_{2} \\
D_{4} \times C_{2} \\
D_{6} \times C_{2} \\
D_{6} \times C_{2} \\
D_{6} \times C_{2} \\
D \times C_{2} \\
D_{6} \times C_{2} \\
D_{6} \times C_{2} \\
D_{6} \times C_{2} \times C_{1} \\
D_{6} \times C_{2} \times C_{2}\end{array}$ & $\begin{array}{l}12 \\
12 \\
12 \\
12 \\
12 \\
24 \\
24 \\
24 \\
24 \\
24 \\
24 \\
48\end{array}$ & 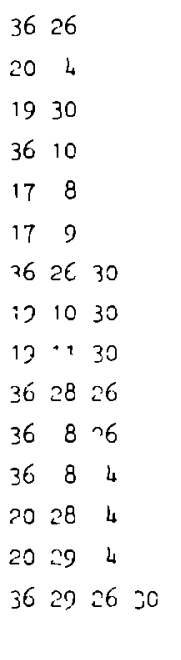 & $\begin{array}{ll}P & A \\
i & A \\
P & A \\
D & A \\
D & A \\
D & A \\
D & A \\
= & A \\
& A \\
\Gamma & A \\
D & A \\
P & A \\
= & A \\
P & A \\
P & A\end{array}$ & $\begin{array}{l}2 \\
2 \\
r \\
2 \\
L\end{array}$ & $\begin{array}{l}185 \\
187 \\
.89 \\
191 \\
193 \\
195 \\
197 \\
199 \\
201 \\
203 \\
205 \\
207 \\
200 \\
211 \\
213\end{array}$ \\
\hline
\end{tabular}




\begin{tabular}{|c|c|c|c|c|c|c|c|}
\hline $\begin{array}{l}\text { System } \\
\text { table II }]\end{array}$ & $\begin{array}{l}\text { Geometz ic } \\
\text { class }\end{array}$ & $\begin{array}{l}\text { Isomorphism } \\
\text { class } \\
{[\text { table III }]}\end{array}$ & Order & $\begin{array}{l}\text { Generators } \\
{[\text { table IV }]}\end{array}$ & $\begin{array}{l}\text { Arithmetic classes } \\
{\left[\begin{array}{c}\text { table } \mathrm{V}] \\
\end{array}\right.}\end{array}$ & $\begin{array}{l}\text { Number } \\
\text { arithm. } \\
\text { el. }\end{array}$ & $\begin{array}{l}\text { Numbering } \\
\text { arithm. cl. }\end{array}$ \\
\hline 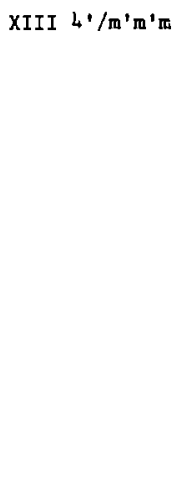 & $\begin{array}{l}4^{\prime}=5 \\
4{ }^{\prime} / \mathrm{m}^{\prime} \\
4^{\prime} 22^{\prime}=42^{\prime} \mathrm{m}^{\prime} \\
4^{\prime} \mathrm{mm}^{\prime}=42 \mathrm{~m} \\
4^{\prime} / \mathrm{m}^{\prime} \mathrm{m}^{\prime} \mathrm{m}\end{array}$ & $\begin{array}{l}C_{4} \\
C_{4} \\
C_{4} \times C_{2} \\
D_{4} \\
D_{4} \\
D_{4} \\
D_{4} \\
D_{4} \\
D_{4} \\
D_{4} \\
D_{4} \\
D_{4} \times C_{2} \\
D_{4} \times C_{2}\end{array}$ & $\begin{array}{l}4 \\
8 \\
8\end{array}$ & $\begin{array}{lrl}14 & \\
13 & & \\
14 & 4 & \\
14 & 11 \\
14 & 12 & \\
13 & 9 \\
13 & 7 \\
14 & 29 \\
13 & 11 \\
14 & 27 & \\
13 & 12 & \\
14 & 9 & 4 \\
14 & 7 & 4\end{array}$ & 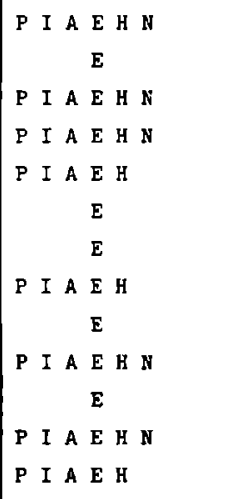 & $\begin{array}{r}7 \\
6 \\
13\end{array}$ & $\begin{array}{l}215 \\
221 \\
222 \\
228 \\
234 \\
239 \\
240 \\
241 \\
246 \\
247 \\
253 \\
254 \\
260\end{array}$ \\
\hline XII $4 / \operatorname{manm} 11$ & 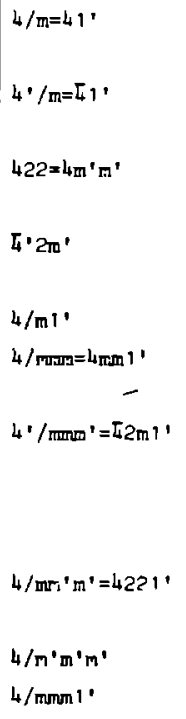 & $\begin{array}{l}C_{4} \times C_{2} \\
C_{4} \times C_{2} \\
C_{4} \times C_{2} \\
C_{4} \times C_{2} \\
D_{4} \\
D_{4} \\
D_{4} \\
D_{4} \\
C_{4} \times C_{2} \times C_{2} \\
D_{4} \times C_{2} \\
D_{4} \times C_{2} \\
D_{4} \times C_{2} \\
D_{4} \times C_{2} \\
D_{4} \times C_{2} \\
D_{4} \times C_{2} \\
D_{4} \times C_{2} \\
D_{4} \times C_{2} \\
D_{4} \times C_{2} \\
D_{4} \times C_{2} \times C_{2}\end{array}$ & $\begin{array}{c}8 \\
8 \\
8 \\
8 \\
\\
16 \\
16 \\
16\end{array}$ & 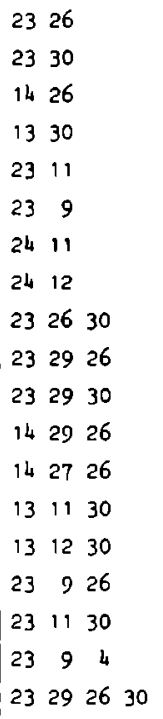 & 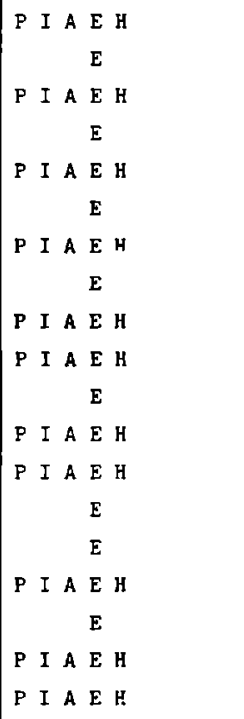 & $\begin{array}{l}6 \\
6 \\
6 \\
6 \\
5 \\
6 \\
12\end{array}$ & $\begin{array}{l}265 \\
270 \\
271 \\
276 \\
277 \\
282 \\
283 \\
288 \\
289 \\
294 \\
299 \\
300 \\
305 \\
310 \\
311 \\
312 \\
317 \\
318 \\
323\end{array}$ \\
\hline$x v m^{\prime} 7 m$ & $\begin{array}{l}23 \\
m^{\prime} 3 \\
4 \cdot 32 \\
43 m \\
m^{\prime} 3 m\end{array}$ & $\begin{array}{l}T \\
T \times C_{2} \\
0 \\
0 \\
0 \times C_{2}\end{array}$ & $\begin{array}{l}12 \\
24 \\
24 \\
24 \\
48\end{array}$ & $\begin{array}{lrr}16 & 3 & \\
16 & 3 & 2 \\
14 & 43 & \\
13 & 43 & \\
14 & 43 & 2\end{array}$ & 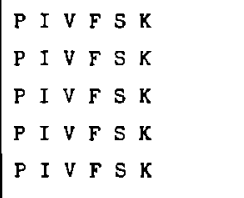 & $\begin{array}{l}6 \\
6 \\
6 \\
6 \\
6\end{array}$ & $\begin{array}{l}328 \\
334 \\
340 \\
346 \\
352\end{array}$ \\
\hline$X I V m 3 m)$ & $\begin{array}{l}m 3 \\
231 \prime \\
m 31 ' \\
432 \\
4 \cdot 3 m^{\prime} \\
m 3 m \\
m^{\prime} 3 m^{\prime} \\
m 3 m^{\prime} \\
4321^{\prime} \\
43 m 1^{\prime} \\
m 3 m m^{\prime}\end{array}$ & $\begin{array}{l}T \times C_{2} \\
T \times C_{2} \\
T \times C_{2} \times C_{2} \\
0 \\
0 \\
0 \times C_{2} \\
0 \times C_{2} \\
0 \times C_{2} \\
0 \times C_{2} \\
0 \times C_{2} \\
0 \times C_{2} \times C_{2}\end{array}$ & $\begin{array}{l}24 \\
24 \\
48 \\
24 \\
24 \\
48 \\
48 \\
48 \\
48 \\
48 \\
96\end{array}$ & 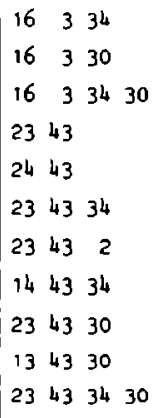 & 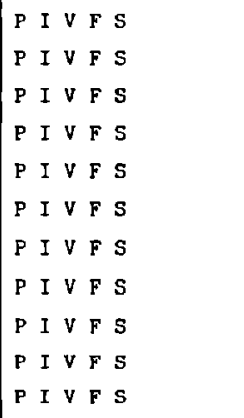 & $\begin{array}{l}5 \\
5 \\
5 \\
5 \\
5 \\
5 \\
5 \\
5 \\
5 \\
5 \\
5\end{array}$ & $\begin{array}{l}358 \\
363 \\
368 \\
373 \\
378 \\
383 \\
388 \\
393 \\
398 \\
403 \\
408\end{array}$ \\
\hline
\end{tabular}




\section{Table II}

\section{Four-dinersional Bravais classes with a holohedry corresponding to a GM pount group}

\begin{tabular}{|c|c|c|c|c|c|c|c|c|}
\hline ystem No. & System Group & Order & Metric & tenso: & & & $\begin{array}{l}\text { Non-equivalent } \\
\text { centerings } \\
\text { (tabie V) }\end{array}$ & $\begin{array}{l}\text { Nurber of free } \\
\text { parameters }\end{array}$ \\
\hline I & $\overline{1}$ & 2 & $\left.\right|^{a_{1}^{2}}$ & $\begin{array}{c}a_{1} \cdot a_{2} \\
a_{2}^{2}\end{array}$ & $\begin{array}{c}a_{1} \cdot a_{3} \\
a_{2} \cdot a_{3} \\
a_{3}^{2}\end{array}$ & $\left.\begin{array}{c}a_{1} \cdot a_{4} \\
a_{2} \cdot a_{4} \\
a_{3} \cdot a_{4} \\
a_{4}^{2}\end{array}\right)$ & $P$ & 10 \\
\hline II & $\bar{i}_{1}$, & 4 & $a_{1}^{2}$ & $\begin{array}{r}0 \\
a_{2}^{2}\end{array}$ & $\begin{array}{c}0 \\
a_{2} \cdot a_{3} \\
a_{3}^{2}\end{array}$ & $\begin{array}{c}0 \\
a_{2} \cdot \alpha_{6} \\
a_{3} \cdot a_{4} \\
a_{4}^{2}\end{array}$ & $\mathrm{P}, \mathrm{I}$ & 7 \\
\hline III & $2 / m^{\prime}$ & 4 & $\mathbf{a}_{1}^{2}$ & $\begin{array}{c}a_{1} \cdot a_{2} \\
a_{2}^{2}\end{array}$ & $\begin{array}{r}0 \\
0 \\
a_{3}^{2}\end{array}$ & $\begin{array}{c}c \\
0 \\
a_{3} \cdot a_{4} \\
a_{4}^{2}\end{array} \mid$ & $P, I, C$ & 6 \\
\hline IV & $2 / \pi_{1} 1^{\prime}$ & 8 & $a_{1}^{2}$ & $\begin{array}{r}0 \\
\mathbf{a}_{2}^{2}\end{array}$ & $\begin{array}{r}0 \\
0 \\
\theta_{3}^{2}\end{array}$ & $\begin{array}{c}0 \\
0 \\
a_{3} \cdot a_{4} \\
a_{4}^{2}\end{array}$ & $P, B, I, C, F, G$ & 5 \\
\hline$v$ & 6/m'm & 24 & $a_{1}^{2}$ & $\begin{array}{l}l_{1}^{2} a_{1}^{2} \\
a_{1}^{2}\end{array}$ & $\begin{array}{r}0 \\
0 \\
a_{3}^{2}\end{array}$ & $\begin{array}{c}0 \\
0 \\
a_{3} \cdot a_{4} \\
a_{4}^{2}\end{array} \mid$ & $\mathbf{P}$ & 4 \\
\hline vI & $y^{\prime} m$ & 12 & $a_{1}^{2}$ & $\begin{array}{l}a a_{1}^{2} \\
a_{1}^{2}\end{array}$ & $\begin{array}{l}a a_{1}^{2} \\
a a_{1}^{2} \\
a_{1}^{2}\end{array}$ & $\begin{array}{c}a_{1} \cdot a_{-} \\
a_{1} \cdot a_{-} \\
a_{1} \cdot a_{-} \\
a_{4}^{2}\end{array} \mid$ & $P(V I P=V P)$ & 4 \\
\hline VII & $L / \pi^{\prime} \pi \pi$ & 16 & $a_{1}^{2}$ & $\begin{array}{l}0 \\
a_{1}^{2}\end{array}$ & $\begin{array}{r}0 \\
0 \\
a_{3}^{2}\end{array}$ & $\begin{array}{c}0 \\
0 \\
a_{3} \cdot a_{4} \\
a_{4}^{2}\end{array} \mid$ & $\because=$ & 4 \\
\hline (viII & $\min 11^{\prime}$ & 16 & $a_{1}^{2}$ & $\begin{array}{l}0 \\
a_{2}^{2}\end{array}$ & $\begin{array}{r}0 \\
0 \\
a_{3}^{2}\end{array}$ & $\begin{array}{c}0 \\
0 \\
0 \\
\mathbf{s}_{4}^{2}\end{array}$ & $\begin{array}{l}P, I, A, E \\
F, \cap, H, S\end{array}$ & 4 \\
\hline$I X$ & $m^{\prime} m^{\prime} m^{\prime}$ & $\theta$ & $\mathbf{e}_{1}^{2}$ & $\begin{array}{l}\alpha \theta_{1}^{2} \\
a_{1}^{2}\end{array}$ & $\begin{array}{l}3 a_{1}^{2} \\
\gamma a_{1}^{2} \\
a_{1}^{2}\end{array}$ & $\begin{array}{l}r a_{1} \\
g_{a_{1}^{2}}^{2} \\
\alpha a_{1}^{2} \\
a_{1}^{2}\end{array} \mid$ & $P(I X P=V I I I K)$ & 4 \\
\hline
\end{tabular}




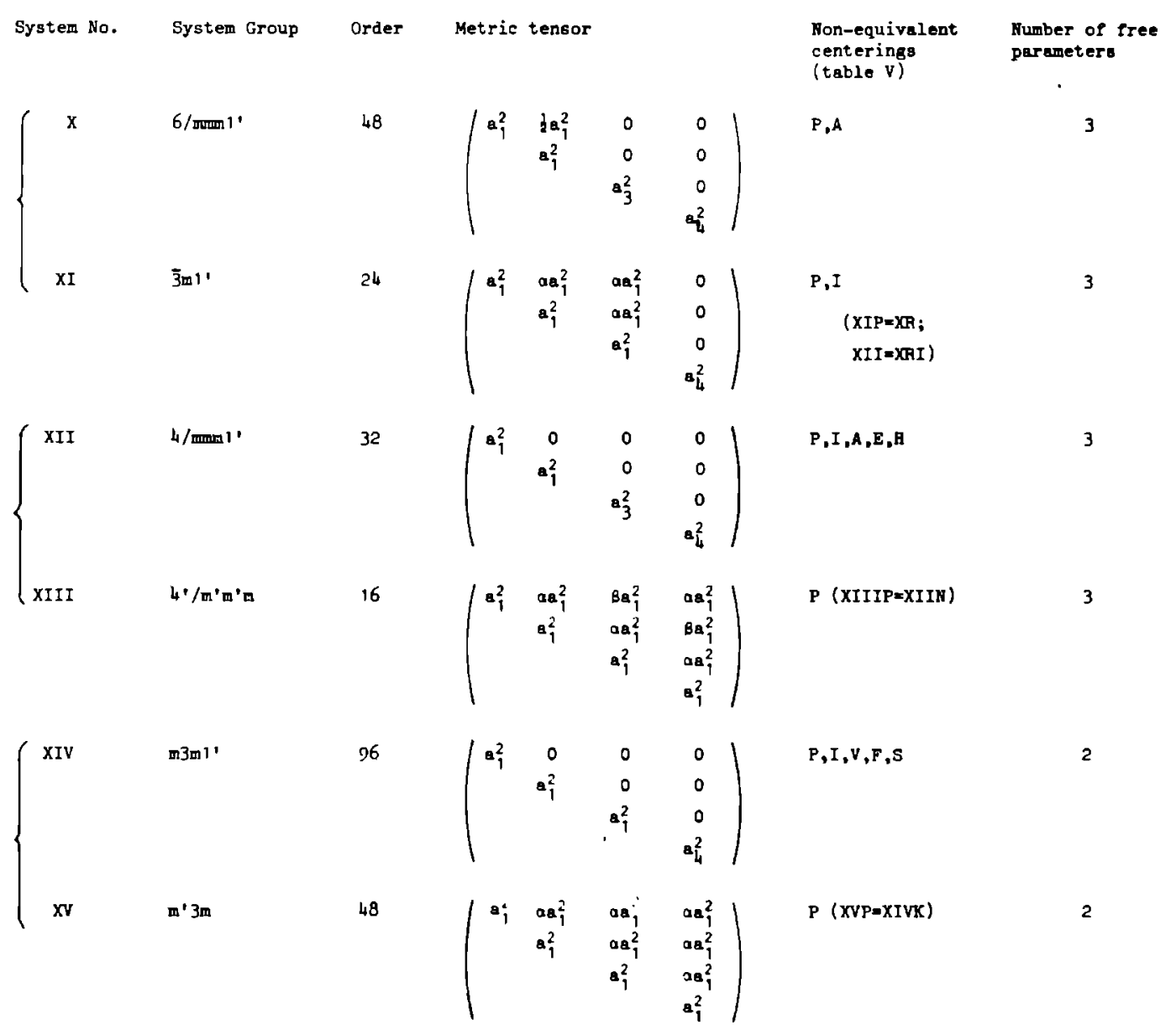

$(a, B, Y \in R)$

Table III

\begin{tabular}{|c|c|c|c|}
\hline & Isomorphism clesses & & \\
\hline $\begin{array}{l}C_{n}(n=1,2,3,4,6) \\
C_{n} \times C_{2} \quad(n=4,6) \\
C_{n} \quad C_{2} \times C_{2} \quad(n=4,6) \\
D_{n}(n=2,3,4,6) \\
D_{n} \times C_{2} \quad(n=2,4,6) \\
D_{n} \times C_{2} \times C_{2} \quad(n=2,4,6)\end{array}$ & $\begin{array}{l}\left\langle a ; a^{1:}=\varepsilon\right\rangle \\
\left.\Leftrightarrow a_{1}, a_{2} ; a_{1}^{n}=a_{2}^{2}=\left(a_{1} a_{2}\right)^{2}=\varepsilon\right\rangle\end{array}$ & $\begin{array}{l}T \\
T \times C_{2} \\
T \times C_{2} \times C_{2} \\
0 \\
0 \times C_{2} \\
0 \times C_{2} \times C_{2}\end{array}$ & $\begin{array}{l}\left\langle\alpha_{1}, \alpha_{2} ; a_{1}^{3}=\alpha_{2}^{2}=\left(a_{1} \alpha_{2}\right)^{3}=c\right\rangle \\
\left\langle\alpha_{1}, \alpha_{2} ; a_{1}^{4}=a_{2}^{3}=\left(a_{1} a_{2}\right)^{2}=\varepsilon\right\rangle\end{array}$ \\
\hline
\end{tabular}




\section{Table IV}

\section{Generators of arithmetic point groups}

+: direct sum; n order; $x$ trace; d determınant; a second invariant ${ }^{*}$

$E=+1 \quad I=-1$

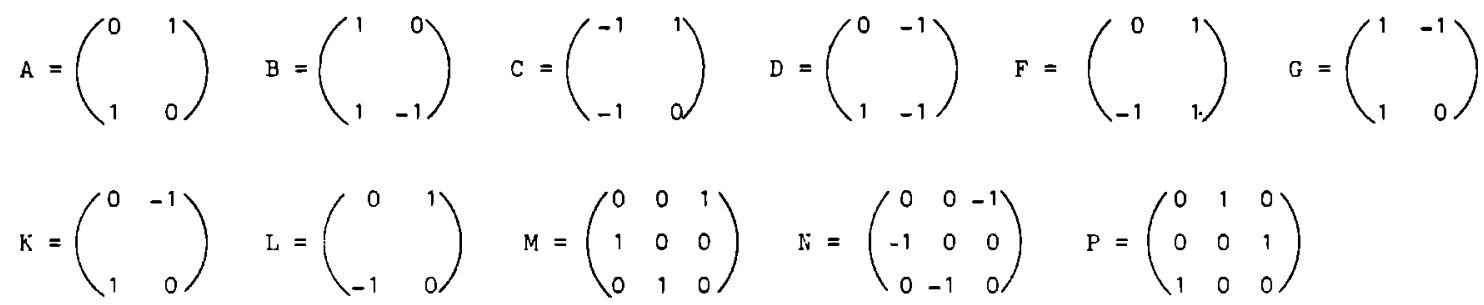

\begin{tabular}{|c|c|c|c|c|c|c|c|c|c|c|c|c|c|c|c|c|c|}
\hline & & $n$ & & $d$ & 0 & & & $\mathrm{n}$ & & $d$ & $c$ & & & $\mathrm{n}$ & r & $d$ & 0 \\
\hline 1. & $\mathrm{E}+\mathrm{E}+\mathrm{E}+\mathrm{E}$ & 1 & 4 & 1 & 6 & 16. & $M+E$ & 3 & 1 & $?$ & 0 & 31. & $\mathrm{~L}+\mathrm{I}+\mathrm{I}+\mathrm{I}$ & 2 & -2 & -1 & 0 \\
\hline 2. & $I+I+I+I$ & 2 & -4 & ' & 6 & 17. & $D+I+I$ & 6 & -1 & -3 & 0 & 32. & $A+I+I$ & 2 & -2 & -1 & 0 \\
\hline 3. & $J+I+E+E$ & 2 & 0 & 1 & -2 & 18. & $N+I$ & 6 & -1 & 1 & 0 & 33. & $3+I+I$ & 2 & -2 & -1 & 0 \\
\hline 4. & $\mathrm{E}+\mathrm{E}+\mathrm{I}+\mathrm{I}$ & 2 & 0 & 1 & -2 & 19. & $\mathrm{D}+\mathrm{I}+\mathrm{E}$ & 6 & 1 & -1 & 0 & 34. & $I+I+I+E$ & 2 & -2 & -1 & 0 \\
\hline 5. & $I+E+I+E$ & 2 & 0 & 1 & -2 & 20 & $F+E+I$ & 6 & 1 & -1 & 0 & 35. & $I+I+E+I$ & 2 & -2 & -1 & 0 \\
\hline 6. & $I+E+E+I$ & 2 & 0 & 1 & -2 & 21. & $N+E$ & 6 & 1 & -1 & 0 & 36. & $F+E+E$ & 6 & 3 & 1 & 4 \\
\hline 7. & $\mathrm{E}+\mathrm{I}+\mathrm{E}+\mathrm{I}$ & 2 & 0 & 1 & -2 & 22. & $G+I+E$ & 6 & 1 & -1 & 0 & 37. & $C+I+I$ & 6 & -2 & 1 & 4 \\
\hline 8. & $\mathrm{~B}+\mathrm{E}+\mathrm{I}$ & 2 & 0 & 1 & -2 & 23. & $L+E+E$ & 4 & 2 & 1 & 2 & 38. & $I+E+I+I$ & 2 & -2 & -1 & 0 \\
\hline 9. & $A+E+I$ & 2 & 0 & 1 & -2 & 24 & $K+I+I$ & 4. & -2 & 1 & 2 & 39. & $C+I+I$ & 6 & -1 & 1 & 0 \\
\hline 10 . & $++\Sigma+E$ & 2 & 0 & 1 & -2 & 25. & $I+E+E+E$ & 2 & 2 & -1 & 0 & 40 & $\mathrm{C}+\mathrm{I}+\mathrm{E}$ & 6 & 1 & -1 & 0 \\
\hline 11. & $A+I+E$ & 2 & 0 & 1 & -2 & 26. & $E+E+I+E$ & 2 & 2 & -1 & 0 & 41. & $F+I+E$ & 6 & 1 & -1 & 0 \\
\hline 12. & $\mathrm{E}+\mathrm{I}+\mathrm{I}+\mathrm{E}$ & 2 & 0 & 1 & -2 & 27. & $E+I+E+E$ & 2 & 2 & -1 & 0 & 42. & $P+E$ & 3 & 1 & 1 & 0 \\
\hline 13. & $K+I+E$ & 4 & 0 & -1 & 0 & 28. & $B+E+E$ & 2 & 2 & -1 & 0 & & & & & & \\
\hline 14. & $\mathrm{~L}+\mathrm{E}+\mathrm{I}$ & 4 & 0 & -1 & 0 & 29. & $A+E+E$ & 2 & 2 & -1 & 0 & & & & & & \\
\hline 15. & $C+E+E$ & 3 & 1 & 1 & 0 & 30. & $E+E+E+I$ & 2 & 2 & -1 & 0 & & & & & & \\
\hline
\end{tabular}

-) The characteristic equation is $1^{4}-x^{\lambda^{3}}+0 \lambda^{2} \pm x \lambda+d=0$. 


\section{Table V}

\section{Centerings of the lattices of table II}

a) centering symbol; b) number of interior points per unit cell; c) interior points;

d) transformation matrix (4.1).

\begin{tabular}{|c|c|c|c|c|c|c|}
\hline $\mathrm{a} b$ & & $d$ & $\mathrm{ab}$ & c & & $d$ \\
\hline I $2(0000)\left(\frac{1}{2} \frac{1}{2} \frac{1}{2} \frac{1}{2}\right)$ & $\frac{1}{2}$ & $\left(\begin{array}{rrrr}1 & 1 & 1 & 1 \\
-1 & 1 & 1 & 1 \\
1 & -1 & 1 & 1 \\
1 & 1 & -1 & 1\end{array}\right)$ & E 2 & $2(0000)\left(\frac{1}{2} \frac{1}{2} 0 \frac{1}{2}\right)$ & $\frac{1}{2}$ & $\left(\begin{array}{rrrr}1 & 1 & 0 & 1 \\
-1 & i & 0 & 1 \\
1 & -1 & 0 & 1 \\
0 & 0 & 2 & 0\end{array}\right)$ \\
\hline$V 2(0000)\left(\frac{1}{2} \frac{1}{2} \frac{1}{2} 0\right)$ & $\frac{1}{2}$ & $\left(\begin{array}{rrrr}1 & 1 & 1 & 0 \\
-1 & 1 & 1 & 0 \\
1 & -1 & 1 & 0 \\
0 & 0 & 0 & 2\end{array}\right)$ & A 2 & $2(0000)\left(00 \frac{1}{2} \frac{1}{2}\right)$ & & $\left(\begin{array}{rrrr}0 & 0 & 1 & 1 \\
0 & 0 & -1 & 1 \\
2 & 0 & 0 & 0 \\
0 & 2 & 0 & 0\end{array}\right)$ \\
\hline C $2(0000)\left(\frac{1}{2} \frac{1}{2} 00\right)$ & $\frac{1}{2}$ & $\left(\begin{array}{rrrr}1 & 1 & 0 & 0 \\
-1 & 1 & 0 & 0 \\
0 & 0 & 2 & 0 \\
0 & 0 & 0 & 2\end{array}\right)$ & F & $4(0000)\left(\frac{1}{2} \frac{1}{2} 00\right)\left(\frac{1}{2} 0 \frac{1}{2} 0\right)\left(0 \frac{1}{2} \frac{1}{2} 0\right)$ & & $\left(\begin{array}{rrrr}1 & 1 & 0 & 0 \\
1 & -1 & 0 & 0 \\
1 & 0 & -1 & 0 \\
0 & 0 & 0 & 2\end{array}\right)$ \\
\hline B $2(0000)\left(\frac{1}{2} 0 \frac{1}{2} \frac{1}{2}\right)$ & $\frac{1}{2}$ & $\left(\begin{array}{rrrr}1 & 0 & 1 & 1 \\
-1 & 0 & 1 & 1 \\
1 & 0 & -1 & 1 \\
0 & 2 & 0 & 0\end{array}\right)$ & $\mathrm{H}$ & $4(0000)\left(\frac{1}{2} \frac{1}{2} 0 \frac{1}{2}\right)\left(00 \frac{1}{2} \frac{1}{2}\right)\left(\frac{1}{2} \frac{1}{2} \frac{1}{2} 0\right)$ & & $\left(\begin{array}{rrrr}1 & 1 & 0 & 1 \\
1 & -1 & 0 & 1 \\
-1 & 1 & 0 & 1 \\
0 & 0 & -1 & 1\end{array}\right)$ \\
\hline G $4(0000)\left(\frac{1}{2} 0 \frac{1}{2} 0\right)\left(0 \frac{1}{2} 0 \frac{1}{2}\right)\left(\frac{1}{2} \frac{1}{2} \frac{1}{2} \frac{1}{2}\right)$ & $\frac{1}{2}$ & $\left(\begin{array}{rrrr}1 & 0 & 1 & 0 \\
-1 & 0 & 1 & 0 \\
0 & 1 & 0 & 1 \\
0 & 1 & 0 & -1\end{array}\right)$ & & 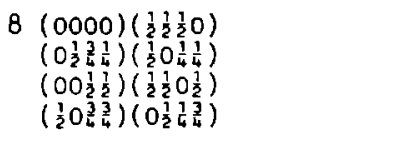 & $\frac{1}{4}$ & $\left(\begin{array}{rrrr}2 & 0 & 1 & 1 \\
0 & 2 & -1 & 1 \\
-2 & 0 & 1 & 1 \\
0 & -2 & -1 & 1\end{array}\right)$ \\
\hline R $3(0000)\left(\frac{1}{3} \frac{1}{1} \frac{1}{1}\right)\left(\frac{2}{3} 3 \frac{2}{3} \frac{2}{3}\right)$ & $\frac{1}{3}$ & $\left(\begin{array}{rrrr}1 & 1 & 1 & 0 \\
-2 & 1 & 1 & 0 \\
1 & -2 & 1 & 0 \\
0 & 0 & 0 & 3\end{array}\right)$ & s & $\begin{aligned} & 8(0000)\left(\frac{1}{2} 0 \frac{1}{2} 0\right) \\
&\left(\frac{1}{2} 00 \frac{1}{2}\right)\left(0 \frac{1}{2} \frac{1}{2} 0\right) \\
&\left(0 \frac{1}{2} 0 \frac{1}{2}\right)\left(00 \frac{1}{2} \frac{1}{2}\right) \\
&\left(\frac{1}{1} \frac{1}{2} 00\right)\left(\frac{1}{2} \frac{1}{2} \frac{1}{2} \frac{1}{2}\right)\end{aligned}$ & $\frac{1}{2}$ & $\left(\begin{array}{rrrr}1 & 1 & 0 & 0 \\
-1 & 1 & 0 & 0 \\
-1 & 0 & 1 & 0 \\
-1 & 0 & 0 & 1\end{array}\right)$ \\
\hline 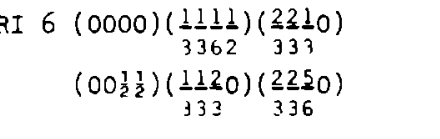 & $\frac{1}{6}$ & $\left(\begin{array}{rrrr}0 & 0 & 3 & 3 \\
-2 & -2 & 1 & 3 \\
4-2 & 1 & 3 \\
-2 & 4 & 1 & 3\end{array}\right)$ & K 16 & 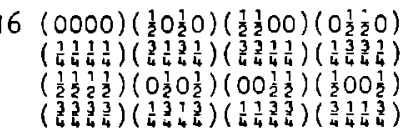 & $\frac{1}{4}$ & $\left(\begin{array}{rrrr}1 & 1 & 1 & 1 \\
-1 & -1 & 1 & 1 \\
-1 & 1 & -1 & 1 \\
1 & -1 & -1 & 1\end{array}\right)$ \\
\hline
\end{tabular}




\section{SAMENVATTING}

Drie-dimensionale magnetische kristallografische groepen zijn al vele jaren bestudeerd en toegepast. We behandelen hier een generalisatie van dit begrip tot symmetriegroepen, waarin ook diskrete tijdtranslaties optreden. Dit leidt tot de bestudering van vier-dimensionale kristallografische groepen in Euklidische, Minkowski- en Galilei-ruimten en in het produkt van een drie-dimensionale Euklidische ruimte met een één-dimensionale. In deze ruimten worden kristallografische begrippen gedefinieerd als ruimte(-tijd-) groep, puntgroep, systeemgroep, arithmetische en geometrische kristalklasse, Bravaisklasse en systeem van roosters. In feite kan men al deze begrippen definieren in $n+1$-dimensionale ruimten voor willekeurige $n$. Waar mogelijk zullen we uitspraken doen voor willekeurige $n$.

In deel $I$ wordt een discussie gegeven van de definities. Zoals in het Euklidische geval, treden ruimte-tijd-groepen op in $\left(K, z^{n+1}, \phi\right)$-uitbreidingen, warbij $K$ een kristallografische puntgroep is en $\phi$ een monomorphisme $\phi: K \rightarrow G L(n+1, Z)$. Omdat het nog niet bekend is onder welke voorwaarden een dergelijke uitbreiding geinterpreteerd kan worden als ruimte-tijd-groep (in het Euklidische geval is dit mogelijk voor iedere eindige $K$ ), beperken we de klassifikatie hier tot het geval van eindige $K$. De klassifikatie gebeurt met een isomorfierelatie die rekening houdt met de verschillende soorten translatieelementen.

Voor gegeven geometrische klassen wordt een konstruktieve methode behandeld om alle niet-isomorfe ruimte(-tijd-) groepen te bepalen. Het aantal Bravaisklassen blijkt eindig te zijn in Euklidische en Galilei-ruimten. Het is aftelbaar oneindig in de zogenaamde produktruimte en overaftelbaar in de Minkowski-ruimte. Hetzelfde geldt voor het aantal niet-isomorfe ruimte(-tijd-) groepen.

In deel II wordt de bepaling van de niet-isomorfe uitbreidingen behorend bij een arithmetische puntgroep meer in detail besproken. De afleiding van de uitbreidingskondities (volgens $M$. Hall) wordt aangegeven en expliciet uitgevoerd voor uitbreidingen van een willekeurige abelse groep met een groep isomorf met een reële $3+1$-reducibele kristallografische puntgroep. 
Voor uitbreidingen van $\mathrm{z}^{\mathrm{n}}$ is een kennis van deze uitbreidingskondities niet nodig. Voor dit geval wordt een eenvoudige methode gegeven om alle niet-equivalente uitbreidingen voor gegeven arithmetische puntgroep te bepalen. De uiteindelijke formules lijken veel op uitdrukkingen van Zassenhaus. Dit kan worden verklaard uit de isomorfie tussen $\mathrm{H}_{\phi}^{2}\left(K, Z^{n}\right)$ en $H_{\phi}^{l}\left(K, R^{n} / Z^{n}\right)$ voor eindige $K$. Verder worden relaties gegeven tussen verschillende manieren om een uitbreiding vast te leggen. Tenslotte wordt de expliciete bepaling van de niet-isomorfe uitbreidingen besproken.

In deel III worden voor het geval $n+1=4$ de arithmetische kristalklassen en Bravaisklassen afgeleid, waruit met de methode van deel II de niet-isomorfe ruimtegroepen bepaald kunnen worden. We beperken ons weer tot de Euklidische puntgroepen die reëel 3+1-reducibel zijn. Dit zijn de gegeneraliseerde magnetische (GM) puntgroepen. Er bestaat een één-één-duidige korrespondentie tussen $n+1$-dimensionale GM puntgroepen en n-dimensionale magnetische puntgroepen. Daarom zijn de vier-dimensionale GM puntgroepen bekend. Hiervoor worden de overeenkomende arithmetische puntgroepen bepaald door het bepalen van de invariant gelaten roosters en een discussie van hun arithmetische equivalentie. 


\section{SUMMARY}

Magnetic crystallographic groups, defined in three dimensions, have been studied and applied for several years. Here symmetry groups in which also discrete time translations occur are discussed. This leads to the study of four-dimensional crystallographic groups in Euclidean, Minkowskian and Galilean spaces and in the product of a three-dimensional Euclidean space by a one-dimensional one. In these spaces crystallographic concepts are defined like space(-time) group, point group, system group, arithmetic and geometric crystal class, Bravais class and lattice system. Actually all these concepts may be defined for $n+1$-dimensional spaces with $n$ arbitrary. Whenever this is possible we use this more general point of view.

In part one the various definitions are discussed. As in Euclidean space, space-time groups $\mathrm{G}^{\mathrm{n}+1}$ appear in $\left(\mathrm{K}, \mathrm{z}^{\mathrm{n}+1}, \phi\right)$-extensions with $\mathrm{K}$ a crystallographic point group and $\phi$ a monomorphism $\phi: K \rightarrow G L(n+1, Z)$. As it is not yet known under which conditions groups appearing in such extensions may be interpreted as space-time groups (in the Euclidean case this may be done for finite $K$ ), we limit ourselves here to the case of finite $\mathrm{K}$. The classification arises by identifying space(-time) groups related by an isomorphism which takes into due account the various kinds of translation elements.

For known geometric crystal classes a constructive method to determine all non-isomorphic space(-time) groups is treated. The number of Bravais classes in Euclidean and Galilean space turns out to be finite. It is enumerably infinite in the so-called product space and continuously infinite in the Minkowskian space. The same is true for the number of non-isomorphic space (-time) groups.

In part Two the determination of all non-isomorphic extensions for a given arithmetic point group is treated in some more detail. The derivation of the extension conditions (according to M.Hall) is indicated and these are explicitly given for extensions of an arbitrary abelian group by a group isomorphic with a four-dimensional point group which is $3+1$-reducible over $R$.

For extensions of $\mathrm{z}^{\mathrm{n}}$ a knowledge of these conditions is not necessary. For this case a simple method to determine all non-equivalent extensions is 
discussed. The final formulas bear a close resemblance to formulas given by Zassenhaus. This can be explained by the isomorphism of $\mathrm{H}_{\phi}^{2}\left(K, \mathrm{Z}^{\mathrm{n}}\right)$ and $\mathrm{H}_{\phi}^{l}\left(K, \mathrm{R}^{\mathrm{n}} / \mathrm{Z}^{\mathrm{n}}\right)$ for finite $K$. Relations between various ways of fixing extensions are given. Also the explicit determination of the non-isomorphic extensions is treated.

In part Three the arithmetic crystal classes and Bravais classes for $n+1=4$ are given with the restriction to real $3+1$-reducible Euclidean point groups called generalized magnetic (GM) point groups. The corresponding nonisomorphic space groups then may be determined using the method of part Two. There is a one-to-one correspondence between $n+1$-dimensional GM point groups and $n$-dimensional magnetic point groups. Hence the four-dimensional GM point groups are known. The corresponding arithmetic crystal classes are found by constructing the lattices left invariant by these groups and by discussing their arithmetic equivalence. 


\section{REFERENCES}

PART ONE.

[1] W. Opechowskı and R. Guccione. "Magnetic Symmetry" in "Magnetism IIa", ed. Rado and Suhl, Academic Press, New York (1965).

[2] W. Opechowsks and R. Guccione. "Magnetic groups" (to be published).

[3] E. Ascher and A. Janner "Algebraic aspects of crystallography I", Helv. Phys. Acta 38, $551-572$ (1965).

[4] L. Bieberbach: "Ueber die Bewegungsgruppen der Euklidischen Raume", Math. Ann. 70 , 297-336 (1911), Math. Ann. 72, 400-412 (1912).

[5] T. Janssen. "Generalized magnetic groups" (2 vols.). Reports 2-66 and 16-67, Instituut voor Theoretische Fysika, Katholieke Universiteit, Nijmegen.

16] J.J. Burckhardt: "Bewegungsgnuppen der K nstallographe", Birkhauser, Basel (1947).

[7] M. Hall. "Theory of groups", Ch. 15, Macmillan, New York (1959).

[8] A.G. Kurosh: "Theory of groups", Chelsea, New York (1960)

[9] S. McLane. "Homology", Springer, Berlin (1963).

[10] M. Hall: "Group rings and extensions", Ann. Math. 39, 220-234 (1938).

\section{PART TWO}

11] E. Ascher and A. Janner: "Algebraic aspects of crystallography I", Helv. Phys. Acta 38, 551 1-572 (1965).

[2] E. Ascher and A. Janner. "Algebrac aspects of crystallography II", (to be published).

[3] M. Hall. "The theory of groups", Macmillan, New York (1959).

[4] Part One of the present treatise.

[5] H Zassenhaus "Ueber einen Algonthmus zur Bestımmung der Raumgruppen". Comm. Helv. Math. 21, 117-141 (1948).

I6] G. Fast and T. Janssen. "Non-equivalent four-dımensional generalızed magnetıc space-tıme groups", Tech. Rep., Inst. Theor. Fysika, Nijmegen.

[7] M. Hall "Group rings and extensions", Ann. Math. 39, 220-234 (1938).

[8] W. Magnus. "On a theorem of Marshall Hall", Ann. Math. 40, 764-768 (1939).

[9] C. Curtus and 1. Reiner. "Representation theory of finite groups and associative algebras", Interscience, New York (1962).

[10] N.F.M. Henry and K. Lonsdale. "International tables for X-ray crystallography, Vol. I, Symmetry groups", The Kynoch Press, Burmungham, England (1952).

\section{PART THREE}

[1] E. Ascher and A. Janner. "Algebraic aspects of crystallography I", Helv. Phys. Acta 38, $551-572$ (1965).

[2] Part Two of the present treatise.

[3] Part One of the present treatise.

[4] H. Zassenhaus "On the classification of the finite integral groups of degree 4", (not yet published).

[5] R. Bulow. "Eıne Ableitung der KnstalkJassen $1 \mathrm{~m}_{4}$ mit Hulfe gruppentheoretischer Programme, Diplom Arbett, Kiel (1967).

[6] E.C. Dade "The maxımal finste groups of $4 \times 4$ integral matnces", Illinois J. Math. $\underline{9}$, 99-1 22 (1965).

17] A.C. Hurley: "Finite rotation groups and crystal classes in 4 dimensions", Proc. Camb. Phil. Soc. 47 , 650-661 (1951).

[8] A.C. Hurley, in "Quantum theory of atoms, molecules and the solud state", ed. P.O. Lowdun, Academic Press (1966).

[9] A.C. Hurley, J. Neubuser, H. Wondratschek- "Crystal classes of four-dımensional space R 4 ", Acta Cryst. 22,605 (1967).

[10] A.L. Mackay and G.S. Pawley. "Bravais lattices in four-dımensıonal space", Acta Cryst. 16, 11-19 (1963).

[11] W. Opechowskı and R. Guccione. "Magnetic Symmetry" in "Magnetısm Ila", ed. Rado and Suhl, Academic Press, New York (1965).

[12] E. Ascher and A. Janner "Subgroups of black-and-white point groups", Acta Cryst. 18, 325-330 (1965).

[13] M.J. Buerger. "Elementary crystallography", Ch. 8, John Wiley, New York (1963).

[14] N. Henry and K. Lonsdale: "International tables for X-ray crystallography, Vol. I, Symmetry groups", The Kynoch Press, Burmingham, England (1952) 


\section{Stellingen}

1. Als $K$ een $n$-dimensionale kristallografische puntgroep met kleinste aantal generatoren $\nu$ is en $A$ een $Z K$-moduul, kan een coketen-komplex

$$
0 \rightarrow A^{\mathrm{J}(0)} \stackrel{\pi^{0}}{\rightarrow} A^{\mathrm{J}(1) \stackrel{\pi^{1}}{\rightarrow}} A^{\mathrm{J}(2)} \rightarrow \ldots \ldots \mathrm{A}^{\mathrm{J}(\mathrm{m})} \stackrel{\pi^{\mathrm{m}}}{\rightarrow} \ldots \ldots
$$

met $A^{\mathrm{J}(\mathrm{m})}=A \times A \times \ldots \times A(\mathrm{~J}(\mathrm{~m})$ faktoren) gedefinieerd worden.

Voor $n<4$ en $m<3$ kan de corand-operator $\pi^{m}$ beschreven worden door een $\mathrm{J}(\mathrm{m}+1) \mathrm{j}(\mathrm{m})$ matrux met elementen uit $Z K$ en $\mathrm{J}(\mathrm{m})=\left(\begin{array}{c}\boldsymbol{m}-1 \\ \mathrm{~m}\end{array}\right)$

2. Als een funktie $f(x)$, bekend op equidistante punten, benaderd kan worden door een $\mathrm{N}$-de graads polynoom, kan een integraal over deze funktse benaderd worden met

$$
\int_{a}^{b} f(x) d x=\sum_{1=-p}^{N \cdot p} d_{1} f\left(x_{1}\right) \Delta \text { met } x_{1}=a+1 \Delta \text { en } x_{N}=b,
$$

waar de koefficienten $d_{0}, \ldots, d_{N}$ een oplossing vormen van

$$
\sum_{i=-p}^{N-p} i^{k} d_{1}=\frac{1}{k+1} N^{k+1} \quad(0 \leqslant k \leqslant N)
$$

Een analoge uitdrukkıng kan worden _egeven voor de p-de afgeleıde.

3 Een diskrete groep, die voor tedere energe $(E \geq 0)$ een ondergroep is van de symmetriegroep van het waterstofatoom, geeft dezelfde opsplitsing van de nuveaux der gebonden toestanden als de korresponderende ondergroep van 0 (3) zou doen, indien men alleen de rotatie-symmetne in beschouwing zou nemen.

4. Neemt men aan, dat de T-matrix buiten de energeschil slechts langzaam vaneert met de energie, dan is het mogelujk een algebrasche vergeluking op te stellen voor de verstrooung van een deeltje aan een gebonden toestand van twee andere deeitjes.

5. In de klassieke limiet, voor $\Lambda=\left(h^{2} \beta / 2 \pi m\right)^{1 / 2} \rightarrow 0$, gaat de diagramontwikkeling van Lee en Yang over in de klassieke Ursell-Mayer ontwikkeling.

T Janssen, Physuca 26, S 75 (1960).

6. De kromme voor de partièle zuurstofdruk als funktıe van de plaats błj zuurstoftransport, gestımuleerd door de aanwezıgheid van hemoglobine, zoals gegeven door Wyman, is niet in overeenstemming met de randvoorwarden die bij zijn differentiaalverge lujking horen.

J. Wyman, J. Bıl. Chem. 241, 115 (1966).

7. De anisotropie van de susceptibiliteit in bismuth-tellunde kan op eenvoudige wijze verklanrd worden.

A. Van Itterbeek e.a., Physuca 32, 2123 (1966).

8. De wijze, waarop Zak de representatues van de nuet-symmorfe ruimtegroepen afleidt, is onjuist.

J. Zak, J Math. Phys. 1, 165 (1960). 



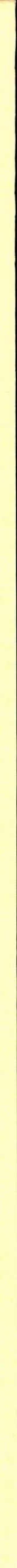


